(c) 2004 International Press

Adv. Theor. Math. Phys. 8 (2004) 603-696

\title{
The Hagedorn/Deconfinement Phase Transition in Weakly
}

\section{Coupled Large $N$ Gauge Theories}

\author{
Ofer Aharony ${ }^{\mathrm{a}}$, Joseph Marsano ${ }^{\mathrm{b}}$, Shiraz Minwalla ${ }^{\mathrm{b}}$, \\ Kyriakos Papadodimas $^{b}$ and Mark Van Raamsdonk ${ }^{c, d}$

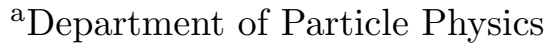 \\ Weizmann Institute of Science, Rehovot 76100, Israel \\ ${ }^{\mathrm{b}}$ Jefferson Physical Laboratory \\ Harvard University, Cambridge, MA 02138 \\ ${ }^{\mathrm{c}}$ Department of Physics \\ Stanford University, Stanford, CA 94305 \\ ${ }^{\mathrm{d}}$ Department of Physics and Astronomy \\ University of British Columbia \\ Vancouver, BC, V6T 1Z1, Canada
}

We demonstrate that weakly coupled, large $N, d$-dimensional $S U(N)$ gauge theories on a class of compact spatial manifolds (including $S^{d-1} \times$ time) undergo deconfinement phase transitions at temperatures proportional to the inverse length scale of the manifold in question. The low temperature phase has a free energy of order one, and is characterized by a stringy (Hagedorn) growth in its density of states. The high temperature phase has a free energy of order $N^{2}$. These phases are separated either by a single first order transition that generically occurs below the Hagedorn temperature or

e-print archive: http://lanl.arXiv.org/abs/hep-th/0310285 
by two continuous phase transitions, the first of which occurs at the Hagedorn temperature. These phase transitions could perhaps be continuously connected to the usual flat space deconfinement transition in the case of confining gauge theories, and to the Hawking-Page nucleation of $A d S_{5}$ black holes in the case of the $\mathcal{N}=4$ supersymmetric Yang-Mills theory. We suggest that deconfinement transitions may generally be interpreted in terms of black hole formation in a dual string theory. Our analysis proceeds by first reducing the Yang-Mills partition function to a $(0+0)$-dimensional integral over a unitary matrix $U$, which is the holonomy (Wilson loop) of the gauge field around the thermal time circle in Euclidean space; deconfinement transitions are large $N$ transitions in this matrix integral.

\section{Contents}

1 Introduction $\quad 606$

$\begin{array}{lll}2 & \text { Background } & 611\end{array}$

2.1 Hagedorn behavior in string theory . . . . . . . . . . . 611

2.2 Deconfinement transitions in gauge theories . . . . . . . . 614

2.3 Hagedorn versus deconfinement . . . . . . . . . . . . . . 617

2.4 Large $N$ deconfinement on compact spaces . . . . . . . . . . 618

3 Partition function for free Yang-Mills theory on a compact space

3.1 Two matrix harmonic oscillators give Hagedorn behavior for large $N \ldots \ldots \ldots \ldots \ldots$. . . . . . . . . . . . . . . . . .

3.2 Exact partition function for $N=\infty \ldots \ldots 21$

3.3 Exact partition function for free Yang-Mills theory . . . . . . 624

3.4 Generalization to arbitrary chemical potential . . . . . . . 626

$3.5 U(N)$ gauge theories with adjoint matter on $S^{3} \times \mathbb{R} \ldots 626$

4 Path integral derivation of the matrix integral and an order parameter 
4.1 Basic set-up . . . . . . . . . . . . . . . . . 628

4.2 The integration measure . . . . . . . . . . . . . . . 628

4.3 Evaluation of $S_{\text {eff }}$ at one-loop . . . . . . . . . 630

5 Solution of the free Yang-Mills matrix model 633

5.1 Low temperature behavior . . . . . . . . . . . . 635

5.2 Behavior near the transition . . . . . . . . . . . 637

5.3 High temperature behavior . . . . . . . . . . . . 638

5.4 Exact solution for $T>T_{H} \ldots \ldots$. . . . . . . 639

5.5 Perturbative expansion slightly above the Hagedorn temperature . . . . . . . . . . . . . . 6 642

5.6 Summary of thermodynamic behavior . . . . . . . . . . 645

5.7 The Polyakov loop as an order parameter at finite volume . . 646

5.8 Results for specific theories . . . . . . . . . . . . . 648

6 Phase structure at weak coupling $\quad 649$

6.1 General properties of the effective action . . . . . . . . . . 649

6.2 The general form of the effective action in perturbation theory 650

6.3 Possible phase structures at weak coupling . . . . . . . . 651

6.4 Toy model . . . . . . . . . . . . . . . . . 655

6.5 Density of states as a function of energy . . . . . . . . 658

7 Extrapolation to strong coupling and the dual description 662

7.1 Possible phase diagrams for large $N$ Yang-Mills theories . . . 663

7.2 Dual interpretation of the $\mathcal{N}=4 \mathrm{SYM}$ thermodynamics at strong coupling . . . . . . . . . . . . 6669

7.3 Deconfinement and black holes . . . . . . . . . . . 671

7.4 Dual description at a general point in the phase diagram . . . 673 
8 Discussion and future directions

B.1 Counting gauge-invariant states precisely . . . . . . . . 681

B.2 Evaluating single-particle partition functions on spheres . . . 682

C Hagedorn transitions at finite string coupling

685

\section{Introduction}

The thermodynamics of large $N S U(N)$ gauge theories is interesting for at least two different reasons. On the one hand, these theories are believed to share many qualitative features with the finite $N$ non-Abelian gauge theories which are relevant to real-world QCD (or GUTs), including confinement at zero temperature and a deconfinement transition as the temperature is increased. On the other hand, large $N$ gauge theories are believed [80,72] to be dual to weakly coupled string theories, which display Hagedorn behavior (reviewed below) [44] associated with singularities in various thermodynamic quantities. Thus, by studying large $N$ gauge theory thermodynamics, one may hope to achieve a better understanding of both deconfinement transitions in gauge theory and Hagedorn behavior in string theory and, possibly, some relationship between the two.

Unfortunately, asymptotically free gauge theories in Minkowski space are strongly coupled except at very high temperatures, so direct analysis of the thermodynamic behavior usually relies on numerical or lattice techniques. However, by placing the theory on a compact space, one obtains a tunable dimensionless parameter $R \Lambda_{Q C D}$ (where $R$ is the size of the compact space and $\Lambda_{Q C D}$ is the dynamically generated scale of the gauge theory ${ }^{1}$ ) which gives back the Minkowski space theory when it is large, but gives a weakly coupled theory when it is small ${ }^{2}$. In this weak coupling limit the thermo-

\footnotetext{
${ }^{1}$ We use here, and throughout the paper, notations which are appropriate for four dimensional gauge theories. For lower dimensional gauge theories, $\Lambda_{Q C D}$ should be replaced by a scale which is an appropriate power of the 't Hooft coupling constant $g_{Y M}^{2} N$.

${ }^{2} \mathrm{~A}$ similar dimensionless parameter appears also in conformal gauge theories like the $d=4 \mathcal{N}=4$ supersymmetric Yang-Mills theory, which can also be studied at weak
} 
dynamics can be studied at all temperatures in perturbation theory, and this analysis is the goal of the present paper. Specifically, we investigate the thermodynamics of $d$-dimensional $U(N)$ (or $S U(N)$ ) gauge theories with arbitrary adjoint-valued matter fields ${ }^{3}$ on compact spatial manifolds, such as $S^{d-1}$, in the limit of weak coupling. This theory is simple enough to analyze exactly in the large $N$ limit; we will find that it has a surprisingly rich structure with many of the expected features of strongly coupled gauge theory thermodynamics, including stringy Hagedorn behavior and a deconfinement transition.

We should start by emphasizing that even at very weak coupling, $S U(N)$ Yang-Mills theory on a compact manifold behaves very differently from $\left(N^{2}-\right.$ 1 ) copies of the $U(1)$ theory. The excitations of the $U(1)$ theory are arbitrary numbers of photons; in contrast (for example) a single $S U(N)$ gluon is not an allowed excitation on a compact manifold, as gluons source lines of color electric flux. Due to Gauss' law, the only allowed excitations of an $S U(N)$ theory on any compact manifold are combinations of gluons (and any other charged particles in the theory) that are grouped into $S U(N)$ singlets.

As we will see below, the projection onto the singlet sector introduces effective interactions between the gluons. It will turn out that these interactions are negligible at high energies, but they dominate the dynamics at low energies. As a consequence, in the $N \rightarrow \infty$ limit, the gauge theories under study in this paper have at least two distinct phases. The lowest temperature phase (dominated by states with $E / N^{2} \ll 1 / R$ ) has a strongly stringy flavor; it is characterized (see [77, 73] for the free theory) by a Hagedorn-like density of states $\rho(E)$ that grows exponentially with energy, $\rho(E) \propto e^{E / T_{H}}$ with $T_{H}=C / R$, where $C$ is a constant depending on the matter content of the theory and on the shape of the compactification manifold. On the other hand, the high temperature phase (dominated by states with $E / N^{2} \gg 1 / R$ ) behaves qualitatively like a gas of free particles; the free energy $F$ takes the form $F=N^{2} f(T)$, where $f(T) \propto T^{d}$ for $T \gg 1 / R$. In some cases these two phases may be separated by a more mysterious intermediate temperature phase.

The free gauge theory undergoes precisely one phase transition as a function of the temperature; this transition is of first order and it occurs precisely at the Hagedorn temperature $T_{H}$. As far as we know, this result was first found by [46] in a specific case, and was derived more generally in a beauti-

coupling.

${ }^{3}$ Our methods can be easily generalized to include also matter fields in other representations. 
ful paper by Sundborg [77]. ${ }^{4}$ For finite $N$, this phase transition is smoothed out, as it must be for any theory with a finite number of degrees of freedom at finite volume.

Turning on a small 't Hooft coupling $\lambda \equiv g_{Y M}^{2} N$ qualitatively alters the behavior of the free gauge theory. It turns out that the interacting theory displays one of two possible behaviors, depending on the sign of a coefficient determined by two-loop and three-loop vacuum diagrams. When varying the temperature, this theory either undergoes a single first order transition below the Hagedorn temperature, or it undergoes two continuous phase transitions, one at the Hagedorn temperature and the second one slightly above it. Note that, in the first case, the phase transition shields the Hagedorn spectrum of the theory in the sense that there is no temperature at which string excitations of arbitrarily high energy dominate the partition function. In the second case the first of the two phase transitions is truly Hagedorn-like; the singularities in the neighborhood of the phase transition directly encode the high-energy density of states of stringy oscillators. ${ }^{5}$

We derive the results described in the previous paragraphs by reducing the thermal partition function to an integral over a single unitary $U(N)$ (or $S U(N))$ matrix

$$
Z(\beta)=\int[d U] \exp \left[-S_{e f f}(U)\right]
$$

where $U=e^{i \beta \alpha}$ ( $\alpha$ is the zero mode of $A_{0}$ on $\mathcal{M} \times S^{1}, \mathcal{M}$ is the spatial manifold in question, and $\beta \equiv 1 / T)$. The mode $\alpha$ is the lightest mode ${ }^{6}$ of the gauge theory on $\mathcal{M} \times S^{1} ; S_{\text {eff }}(U)$, the quantum effective action for this light mode, may be computed by integrating out all other fields in the theory. This procedure (integrating out the heavy modes) may be explicitly performed in perturbation theory, generating an expression for $S_{\text {eff }}(U)$ as a power series in the 't Hooft coupling $\lambda$. The lowest term in this power series, corresponding to the free gauge theory, is particularly easy to compute, either by evaluating one-loop vacuum diagrams or by explicitly counting gauge-invariant states in free Yang-Mills theory using a projection onto the

\footnotetext{
${ }^{4}$ Sundborg's results do not seem to have received wide attention; in particular, we learned of his paper only after we had independently rederived all of its results, which have substantial overlap with section 3 and parts of section 5 of our paper.

${ }^{5}$ This is consistent with the general arguments of [21,67], that a large $N$ second order deconfinement transition must always be accompanied by a Hagedorn-like spectrum and occur at the Hagedorn temperature.

${ }^{6}$ We assume that there are no additional zero modes for any of the fields. This is not always true, since, for instance, the gauge field may have additional zero modes when the compactification manifold is not simply connected. We will not discuss such examples here.
} 
singlet sector of the theory. ${ }^{7}$ For the free theory we obtain an expression (of the form (1.1)) for $Z(\beta)$ which is exact, even at finite $N$, and applies to any gauge theory with any matter content. The computation of higher order terms is straightforward in principle but tedious in practice.

Having computed $S_{\text {eff }}$ we proceed to evaluate (1.1) in the usual manner, by changing the integration variable to the eigenvalues $e^{i \theta}$ of $U$. For the adjoint theories we consider, the resulting effective action in the free limit is simply the sum over a pairwise potential between the eigenvalues with a temperature-independent repulsive term and an attractive term that increases from zero to infinite strength as the temperature increases from zero to infinity. In the large $N$ limit, the integral is determined by a saddle point characterized by a density of eigenvalues $\rho(\theta)$ that minimizes the effective action. At sufficiently low temperatures, the repulsive term dominates and $\rho(\theta)$ is constant on the circle. At high enough temperatures, the attractive term forces the eigenvalues to bunch together and $\rho(\theta)$ vanishes outside a narrow interval on the circle [77]. In the free theory, these two regimes are separated by a first order transition at the Hagedorn temperature in which the eigenvalue distribution jumps discontinuously from the uniform distribution to a sinusoidal distribution. However, it turns out that depending on the details of the interactions in $S_{\text {eff }}$, the nature of this transition may be modified at arbitrarily weak non-zero coupling.

Based on the general form of $S_{\text {eff }}$, we show that two classes of behavior are possible at weak coupling, as noted earlier. In the first case, we have a single first order phase transition at which the uniform low temperature distribution jumps discontinuously to a non-uniform distribution. This is similar to the behavior in the free theory, but the transition happens strictly below the Hagedorn temperature for non-zero coupling. The second possibility is that the eigenvalue distribution evolves continuously as a function of temperature, changing from a uniform to a non-uniform (but nowhere vanishing) distribution at a first critical temperature, and then developing a gap (on which the distribution vanishes) at a second critical temperature. Consequently, the corresponding theory undergoes two phase transitions as a function of temperature; the first of these is a second order transition at the Hagedorn temperature, while the second is a third order transition closely related to the Gross-Witten phase transition [41] of two dimensional lattice gauge theories.

Which of these two possibilities is realized depends on the sign of a particular coefficient in $S_{\text {eff }}$ which appears to depend on the details of the field content and interactions of the theory in question. We are currently

\footnotetext{
${ }^{7}$ This second method was already used to derive similar expressions in $[46,77]$.
} 
involved in a computation of this coefficient for the $d=4$ pure Yang-Mills theory and for the $d=4 \mathcal{N}=4$ supersymmetric Yang-Mills (SYM) theory; we hope to report the result of this computation soon [3].

The phase transition at (or near) the Hagedorn temperature has a natural interpretation in the gauge theory ${ }^{8}$; it is simply a deconfining transition. The low-temperature phase can be thought of as a gas of singlet 'glueballs', with the free energy scaling as $N^{0}$, while the high temperature phase, with the free energy scaling as $N^{2}$, is a plasma of gluons (and other particles). Indeed, the traditional deconfinement order parameter, the Polyakov loop in the fundamental representation $\frac{1}{N}\left\langle\operatorname{tr}\left(\mathrm{Pe}^{\mathrm{i} \oint \mathrm{A}_{0}}\right)\right\rangle \simeq \frac{1}{N}\langle\operatorname{tr}(\mathrm{U})\rangle$, is a good order parameter for the phase transitions described in this paper. It is zero in the confining phase, and non-zero in the deconfining phase ${ }^{9}$.

The phase transitions we discuss in this paper could potentially be continuously related to several other interesting phase transitions - at least, the values of all the order parameters are consistent with such a continuous relation. In the case of the $3+1$-dimensional $\mathcal{N}=4 S U(N) \mathrm{SYM}$ theory compactified on a sphere, our analysis applies to the deconfinement transition at weak 't Hooft coupling; the deconfinement transition at strong coupling is related [86] by the AdS/CFT correspondence [60] to the HawkingPage transition [48] of gravitational theories on asymptotically anti-de Sitter $(A d S)$ spaces. For asymptotically free gauge theories, our weakly coupled results apply when the scale $R$ of the compactification manifold is much smaller than the strong coupling scale $1 / \Lambda_{Q C D}$. However, when we take $R \Lambda_{Q C D} \gg 1$, our phase transitions could turn into the infinite volume deconfinement transition.

We propose a set of phase diagrams for gauge theories as a function of coupling ( $\lambda$ or $R \Lambda_{Q C D}$, respectively) and temperature that interpolate between our weakly coupled results and the known strong coupling behavior. We are led to speculate on a dual interpretation of deconfinement transitions; in particular, we conjecture that deconfinement transitions are always associated with black hole formation in a dual string theory. We argue that the mysterious intermediate temperature phase associated with second order deconfinement transitions (if such a phase is realized in some theory) would

\footnotetext{
${ }^{8}$ As we have described above, in some situations the theory undergoes two phase transitions upon raising the temperature. The discussion in this paragraph applies to the first of these.

${ }^{9}$ Strictly speaking, the Polyakov loop is zero in both phases as flux conservation forbids placing a single quark on a compact space. In $\S 5.7$ we actually define our order parameter more carefully; it is either $\frac{1}{N}\langle\operatorname{tr}(\mathrm{U})\rangle$ in an infinitesimal deformation of the gauge theory corresponding to an infinitesimal Higgsing of the theory, in the limit that the deformation goes to zero, or we can use $\frac{1}{N^{2}}\left\langle\operatorname{tr}(\mathrm{U}) \operatorname{tr}\left(\mathrm{U}^{\dagger}\right)\right\rangle$.
} 
be dual to a string theory in a background dominated by a strange new type of stable black hole.

The paper is organized as follows. We begin in $\S 2$ with a review of some of the relevant background concerning Hagedorn behavior in string theory, deconfinement transitions, and the relation between them. In $\S 3$, we show that large $N$ free gauge theories have a Hagedorn-like behavior of their spectrum and provide a first derivation of the matrix model expression for the thermal partition function of compactified gauge theories by explicitly summing over gauge-invariant states. In $\S 4$, we derive the same expression by using a path integral formalism. In $\S 5$, we describe the solution of this matrix model, exhibit the phase transition, and both compute and discuss the relevant order parameters. In $\S 6$, we generalize the path integral derivation of (1.1) to interacting gauge theories and determine the general form of $S_{\text {eff }}$ in perturbation theory. Utilizing these results, we discuss the different possibilities for the phase structure of the theory at weak coupling. In $\S 7$, we suggest extrapolations of our results to strong coupling and propose a set of possible phase diagrams for gauge theories as a function of coupling and temperature. We also speculate on the interpretation of deconfinement transitions in terms of black hole formation in a dual string theory. We end in $\S 8$ with a summary of our results and a discussion of some possible future directions. Three appendices contain useful technical results.

\section{Background}

\subsection{Hagedorn behavior in string theory}

A single free closed string has an infinite number of vibration modes, each of which may be excited to arbitrary level. Each vibrational state of the string corresponds to a distinct particle species in space-time. In fact (for strings in Minkowski space), the number of particle species grows exponentially with their mass.

To be specific, consider a free type II superstring whose worldsheet is the direct sum of the free SCFT on $\mathbb{R}^{d-1,1}$ and a compact unitary SCFT $C^{\prime}$ with central charge $\widehat{c}=10-d$. Let $N(M)$ denote the number of particle species in this theory with mass less than $M$. For $M^{2}$ much bigger than the string tension $1 / 2 \pi \alpha^{\prime}$,

$$
\frac{d N}{d M}=\frac{K e^{2 \pi \sqrt{2 \alpha^{\prime}} M}}{M^{d}}+\text { subleading }
$$


where $K$ is a constant. In the zero coupling limit, each particle species is described by its own free quantum field theory. The finite temperature partition function $Z$ of such a system of free quantum fields is easily computed; the contribution of highly excited string states (particle species of large mass, $\left.\alpha^{\prime} M^{2} \gg 1\right)$ is given by ${ }^{10}$

$$
\ln (Z(T))=K^{\prime} T^{\frac{d-1}{2}} \int d M \frac{e^{M\left(2 \pi \sqrt{2 \alpha^{\prime}}-\frac{1}{T}\right)}}{M^{\frac{d+1}{2}}}
$$

for some constant $K^{\prime}$. Note that $Z(T)$ diverges for $T$ larger than the Hagedorn temperature $T_{H}=\frac{1}{2 \pi \sqrt{2 \alpha^{\prime}}}$. As $T$ approaches this critical temperature from below, the partition function develops a singularity

$$
\ln (Z(T)) \propto \begin{cases}(\delta T)^{\frac{d-1}{2}} \ln (\delta T) & d \text { odd } \\ (\delta T)^{\frac{d-1}{2}} & d \text { even }\end{cases}
$$

where $\delta T=T_{H}-T .{ }^{11}$

It is useful to understand the origin of the divergence in (2.2) from an alternate point of view. Recall that, for any system, the thermal partition function may be computed by the Euclidean partition function with the time direction compactified on a thermal circle $S_{T}^{1}$ of circumference $\beta=1 / T$ (bosons have periodic boundary conditions and fermions have anti-periodic boundary conditions around a thermal circle). For free string theory, this means that $Z(T)$ in $(2.2)$ can be computed by the string theory torus partition function on the Euclidean space-time $\mathbb{R}^{d-1} \times S_{T}^{1} \times C^{\prime}$. If we now take the time direction to be in $\mathbb{R}^{d-1}$, we can reinterpret $Z(T)$ as the one-loop contribution to the vacuum energy of string theory on the 9 dimensional space $\mathbb{R}^{d-2} \times S_{T}^{1} \times C^{\prime}$. The one-loop contribution to the vacuum energy of any theory is determined completely by its spectrum; in particular it diverges if the spectrum includes a tachyon. Indeed, superstrings winding a thermal circle an odd number of times have a tachyonic mode when the size of the circle is small enough [6]; this is because modular invariance forces the imposition of the opposite GSO projection, one that projects in the identity operator,

\footnotetext{
${ }^{10}$ Note that $d$ in (2.1) is the number of noncompact directions on the space $\mathbb{R}^{d-1,1} \times C^{\prime}$. It is possible that (2.1) (and the other formulas of this section) apply to more general string backgrounds (for instance with a warped space-time geometry) upon replacing $d$ by $d_{e f f}$, the effective number of noncompact space-time dimensions in such spaces. In particular, $d_{e f f}=1$ on a space like $A d S_{5}$ in global coordinates on which particle propagation is gapped.

${ }^{11}$ Note that the expectation value of the energy density at $T=T_{H}$ is convergent for $d>3$ but diverges when $d \leq 3$. Note also that states of very high energy contribute significantly just below the Hagedorn temperature. Consequently, the properties of the theory at fixed energy $E \sqrt{\alpha^{\prime}}$ (microcanonical ensemble) may differ significantly from the properties of the theory at any fixed temperature (canonical ensemble).
} 
on states of odd winding. Specifically, the ground state of a superstring that winds once around the thermal circle has mass

$$
m_{W}^{2}=\frac{2}{\alpha^{\prime}}\left(\frac{1}{8 \pi^{2} \alpha^{\prime} T^{2}}-1\right)
$$

and becomes tachyonic for $T>T_{H}$, providing an alternate explanation for the divergence of $Z(T)$ for $T>T_{H}$.

For the theory with strictly vanishing string coupling $g_{s}$ the partition function is simply ill-defined at $T>T_{H}$. However, for any finite $g_{s}$, the perturbative analysis of the spectrum breaks down at sufficiently high energies (of order $1 / g_{s}^{2}$ ), so the behavior as $T \rightarrow T_{H}$ is difficult to analyze directly (recall that thermodynamics near the Hagedorn temperature receives nonnegligible contributions from states with arbitrarily high energy). It has been speculated [6] that at any finite coupling, no matter how small, the divergence of the free $Z(\beta)$ above the Hagedorn temperature may be replaced by a phase transition to a new high temperature phase. According to this suggestion, the string mode $W$ winding the thermal circle is the order parameter for this transition $[76,6]$. The phase transition occurs when this mode condenses. This could occur either below the Hagedorn temperature (which is the temperature at which this mode becomes massless), in which case the transition is of first order, or at the Hagedorn temperature, in which case the transition is second order.

Unfortunately, in general we know very little about this conjectured high temperature 'phase' of string theory. In [6] it was argued that, were such a phase transition to occur in flat space, it must be of first order. However, thermodynamics and phase transitions in gravitational systems in flat space are at best approximate notions (see [6] for a nice discussion of this issue). The high temperature 'phase' in flat space seems likely to be ill defined; in this phase energy densities are $\mathcal{O}\left(\frac{1}{g^{2}}\right)$ and would (since the Jeans instability is triggered at these energy densities) appear to involve black holes. As the density of states of Schwarzschild black holes grows even faster than exponential, the existence of such a phase seems problematic.

The picture is much clearer for type IIB string theory on $A d S_{5} \times S^{5}$. At small curvatures, the spectrum of single string states in this theory exhibits a Hagedorn growth, with Hagedorn temperature $T_{H}=\frac{1}{2 \pi \sqrt{2} \alpha^{\prime}}$ and $d_{\text {eff }}=1$ (see $\S 7.2$ ). However, this theory (with temperature conjugate to global time) undergoes a first order phase transition ${ }^{12}$ well below its Hagedorn temperature $[48,86]$. As in flat space, energy densities in the high temperature

\footnotetext{
${ }^{12}$ As the space is effectively compact, the phase transition is sharp only in the limit $g_{s} \rightarrow 0$.
} 
phase are $\mathcal{O}\left(\frac{1}{g^{2}}\right)$, and the high temperature phase is dominated by a big black hole sitting at the center of $A d S$ space. In Euclidean space this black hole may indeed be thought of as a condensate of winding modes ${ }^{13}$, so the Atick-Witten order parameter analysis seems to apply, at least qualitatively, to this situation. ${ }^{14}$ Note that the entropy of big black holes grows relatively slowly with energy in $A d S$ space $\left(S \propto E^{\frac{3}{4}}\right)$, and thermodynamics is well defined at all temperatures.

In this paper we demonstrate that the thermodynamics of weakly coupled large $N, S U(N)$ gauge theories at finite volume (for instance gauge theories on a sphere) has some striking similarities to string thermodynamics on the weakly curved $A d S$ space described above. The theories we study all undergo a phase transition as a function of temperature. The low temperature phase has a Hagedorn growth in the density of states with $d_{\text {eff }}=1$. Finally, the high temperature phase in Euclidean space may be thought of as a condensate of winding modes.

\subsection{Deconfinement transitions in gauge theories}

In this paper we will study the thermodynamics of gauge theories on compact manifolds of size $R$. On taking $R$ to infinity our theories reduce to gauge theories on flat space, whose thermodynamics has been studied extensively (see [40] for a review). In this subsection we review the thermal behavior of gauge theories on $\mathbb{R}^{d-1,1}$. We start with a discussion of confining gauge theories, and at the end of this section we briefly mention the situation with the $d=4 \mathcal{N}=4$ supersymmetric Yang-Mills theory.

It is widely believed that pure $S U(N)$ gauge theories in $d=4$ confine at zero temperature (see [82] and [72] for reviews); the low energy spectrum of the theory may be thought of as a Fock space of interacting glueballs. ${ }^{15}$ Consequently, it is expected that in such theories the low tem-

\footnotetext{
${ }^{13}$ Recall that the Euclidean time cycle is contractible in any black hole. Thus the 'time winding number' symmetry, present in global $A d S$, has been spontaneously broken in the black hole phase, implying that the formation of the black hole must involve the condensation of winding modes.

${ }^{14}$ Note, however, that it is not possible to use this analysis to predict (in this case) the order of the transition, as the Atick-Witten analysis, applied to string theories with a mass gap, could be consistent with either a first order or a second order phase transition, depending on the coupling constants of the theory (see appendix $\mathrm{C}$ ).

${ }^{15}$ Similar expectations hold for gauge theories in lower dimensions, and for four dimensional gauge theories with a sufficiently small amount of charged matter fields; for QCD, the $S U(3)$ gauge theory describing the strong interactions, they are experimentally verified. For simplicity of terminology we will restrict the discussion of this subsection to pure gauge theories in $d=4$.
} 
perature $\left(T \ll \Lambda_{Q C D}\right)$ dynamics may be understood in terms of a sparsely populated thermal bath of glueballs. On the other hand, asymptotic freedom permits a reliable computation of the $T \gg \Lambda_{Q C D}$ behavior of such theories ${ }^{16}$, revealing that this high temperature phase may be understood as a weakly coupled gas of gluons $[24,71]$. The high and low temperature phases appear qualitatively different; we will now review the argument showing that they are distinguished by an order parameter, and so are separated by a phase transition at some intermediate temperature.

It is a defining property of a confining theory (with no fields in the fundamental representation of $S U(N)$ ) that a single external particle in the fundamental representation (a "quark") can be inserted into this theory only at an infinite cost in energy. Heuristically, such a quark forms one end of a QCD string which is infinitely long because it has nowhere else to end. In the low temperature confining phase this long string also has infinite positive free energy. Let $F_{q}(T)$ represent the Yang-Mills free energy at temperature $T$ in the presence of an external quark. It follows from the form of the coupling of an external quark to the gauge fields that $e^{-F_{q}(T) / T}=\langle\mathcal{P}\rangle$, where $\mathcal{P}=\frac{1}{N} \operatorname{tr} \mathrm{P} \exp [-\oint \mathrm{A}]$ is the so called Polyakov loop (sometimes called the Polyakov-Susskind loop [71, 78]), the trace of a Wilson loop around the compactified Euclidean thermal time circle. Thus, $\langle\mathcal{P}\rangle=0$ in the low temperature confining phase.

On the other hand, asymptotic freedom permits reliable computations that establish $\langle\mathcal{P}\rangle \neq 0$ at high enough temperatures. Thus, $\langle\mathcal{P}\rangle$ constitutes an order parameter that sharply distinguishes the low temperature confining phase from the high temperature deconfined phase. From a low temperature point of view, the transition that separates these two phases is associated with the condensation of flux tubes whose effective free energy is driven negative at high enough temperatures (when the energy of these strings is overcome by the entropy of their vibrations).

In the large $N$ limit (with fixed 't Hooft coupling, or equivalently fixed $\left.\Lambda_{Q C D}\right)$ the deconfinement phase transition has yet another order parameter [84]. The confined phase is dominated by gauge-invariant bound states and so its free energy $F(T)$ scales like $N^{0}$ at large $N$. On the other hand, the deconfined phase is described by free gluons, and consequently its free energy scales as $N^{2}$ at large $N$. Thus, in the large $N$ limit, $\lim _{N \rightarrow \infty} F(T) / N^{2}$ constitutes a second order parameter for deconfinement; like the Polyakov loop this new order parameter vanishes in the confined phase but is finite in

\footnotetext{
${ }^{16}$ In infinite volume this statement is far from trivial due to infrared divergences which affect finite-temperature perturbative computations. These problems do not appear at finite volume so they will not be relevant for us.
} 
the deconfined phase.

Next, we turn to a discussion of the order of the deconfinement phase transition. To study the phase transition one may generate an effective action for the Polyakov loop $\mathcal{P}$ by integrating all other fields out of the path integral that generates the Yang-Mills free energy. $\mathcal{P}$ is a complex scalar field on $\mathbb{R}^{3}$; note also that $\mathcal{P} \rightarrow e^{\frac{2 \pi i}{N}} \mathcal{P}$ is a symmetry of the $S U(N)$ Yang-Mills path integral [81] (it is generated by gauge transformations that are single valued only up to an element of the center of the gauge group $\mathbb{Z}_{N}$; such gauge transformations act trivially on the dynamical fields of the pure Yang-Mills theory), which is spontaneously broken in the deconfinement phase transition.

The effective action for $\mathcal{P}$ completely determines the nature and properties of the deconfinement transition. If this transition turns out to be first order then its properties depend on the details of the theory under consideration. However, if the deconfinement transition is of second order then the phase transition point has universal behavior; it must be described by a $\mathbb{Z}_{N}$-invariant fixed point of a complex scalar field on $\mathbb{R}^{3}[79,87]$. Only a small number of fixed points with the required properties are known. For $N=2$ the conditions above uniquely pick out the Wilson Fischer fixed point (the $d=3$ Ising model). For $N=3$ no attractive fixed point with the symmetries listed above is known. For $N \geq 4$ the only known fixed point is $U$ (1) invariant (all operators of the form $\varphi^{k N}$ for integer $k$ and $N \geq 4$ are irrelevant at this fixed point, hence the enhanced symmetry).

The arguments of the paragraph above apply to all confining $S U(N)$ gauge theories with adjoint matter. In the rest of this subsection, however, we will describe what is known about the deconfinement transition of the pure gauge theory on $\mathbb{R}^{3,1}$. Since this phase transition is a strong coupling phenomenon, we cannot study it in perturbation theory, and most knowledge about it comes from lattice simulations. Lattice simulations indicate that the $S U(2)$ deconfinement phase transition is second order and is indeed in the Ising model universality class. The $S U(3)$ deconfinement phase transition is first order, in agreement with the predictions above. However, the $S U(N)$ deconfinement phase transitions for $N \geq 4$ (at least for $N=4,5,6$ ) also appear to be first order; in particular the $U(1)$ invariant $(d=3 \mathrm{XY}$ fixed point) appears not to be attained in pure Yang-Mills ${ }^{17}$ (see, for instance,

\footnotetext{
${ }^{17}$ However, it is entirely possible that this beautiful fixed point describes the phase transition of some other confining gauge theory - say, for instance, $\mathcal{N}=1$ supersymmetric Yang-Mills theory. The critical exponents of this fixed point imply that the singular piece of the free Energy scales like $F \propto(\delta T)^{2.08}$ (where $\delta T=T-T_{c}$ ) upon approaching the phase transition, implying $d_{e f f} \approx 5.16$, see (2.3). It would be fascinating to find a stringy
} 
[58], but see also [68]).

As the final topic in this subsection we review the thermodynamics of the $\mathcal{N}=4 \mathrm{SYM}$ theory in flat space. We focus on the limit $N \rightarrow \infty$ with $\lambda=g_{Y M}^{2} N$ held fixed. As this theory is conformal (and from large $N$ counting) the free energy density $F$ must take the form $F=-\frac{\pi^{2}}{6} N^{2} f(\lambda) T^{4}$. Perturbative computations [30] establish that, at small $\lambda, f(\lambda)=1-\frac{3 \lambda}{2 \pi^{2}}$, while computations using the AdS/CFT correspondence [43] show that, at large $\lambda, f(\lambda)=\frac{3}{4}+\frac{45}{32} \frac{\zeta(3)}{(2 \lambda)^{\frac{3}{2}}}$. It is generally assumed (see, e.g., [52]) that $f(\lambda)$ is analytic on the positive real line (i.e., that one encounters no phase transitions in extrapolating the $\mathcal{N}=4 \mathrm{SYM}$ coupling from zero to infinity; more about this in $\S 7$ ). In summary, the thermodynamics of the $\mathcal{N}=4$ SYM theory in flat space is qualitatively rather boring. The same theory has much more interesting thermodynamics on a sphere as was pointed out in $[86,77]$; we will discuss this issue in great detail below.

\subsection{Hagedorn versus deconfinement}

't Hooft has argued [80] that it should be possible to recast any gauge theory, in the limit of large $N$ with fixed $g_{Y M}^{2} N$, as a string theory with string coupling $g_{s} \propto 1 / N$. If we accept this conjecture, then it is reasonable to expect that the gauge theory deconfinement transition has a dual description as a stringy thermal transition of the sort described in $\S 2.1^{18}$. Indeed, stringy and gauge thermodynamics have many points of similarity $[84,67,6]$; in each case the low temperature phase is a gas of weakly interacting thermally populated stringy particles whose free energy is $\mathcal{O}(1)$ in the relevant coupling constants $\left(\frac{1}{N}\right.$ or $\left.g_{s}\right)$. The phase transition is driven by a condensation of these particles, and is marked by an order parameter that winds around the thermal time circle (Polyakov loop or Euclidean winding string). The high temperature phase has a free energy that scales like the inverse square of the relevant coupling.

If we accept that stringy thermodynamics can be understood in terms of deconfinement, it follows that the relation $\rho(E) \sim \exp \left(E / T_{H}\right)$ must break down in the energy range which is relevant for the high temperature deconfined phase. We can explain this breakdown as follows. In the high temperature phase of asymptotically free gauge theories, the density of states is approximately that of a $3+1$ dimensional free field theory with $N^{2}$ degrees

interpretation or confirmation of this result.

${ }^{18}$ As we have commented in $\S 2.1$, this transition may occur below the Hagedorn temperature. 
of freedom on a space of volume $V, \rho(E) \sim \exp \left(E^{3 / 4} N^{1 / 2} V^{1 / 4}\right)$. A crude estimate of the energy at the point of transition between the two phases may be obtained by equating these two formulas for $\rho(E)$, yielding $E \sim N^{2} V T_{H}^{4}$. Note that this energy is proportional to $N^{2} \sim 1 / g_{s}^{2}$. Gravitational effects are large at these energies for every $g_{s}$, no matter how small, explaining the failure of the free string estimate.

So far we lack a quantitative demonstration that large $N$ gauge thermodynamics exhibits sharp stringy features ${ }^{19}$. The deconfinement transition in flat space occurs at strong coupling and has so far resisted efforts at quantitative analysis. In this paper we will make progress on this important problem by analyzing confining gauge theories on compact spaces ${ }^{20}$ of size $R$ rather than flat space, taking $R \Lambda_{Q C D} \ll 1$. The thermal behavior of such a theory may be computed reliably at all values of the temperature; we will demonstrate that it displays stringy features.

\subsection{Large $N$ deconfinement on compact spaces}

As we have argued at the end of the previous subsection, there are several good motivations to study the thermodynamics of Yang-Mills theories on compact manifolds. The AdS/CFT correspondence forces us to address this problem. In a confining theory the finite size of the manifold cuts off the running of the coupling at the scale $1 / R$, introducing a dimensionless coupling constant into the problem.

In the case of a conformal theory like the $\mathcal{N}=4$ SYM theory the new scale permits nontrivial temperature dependence of thermodynamic quantities. In this subsection we will take a first look at the thermal behavior of gauge theories on such spaces.

Clearly, the thermodynamical properties of an $S U(N)$ gauge theory on a compact space of size $R \gg 1 / \Lambda_{Q C D}$ closely resemble those of the theory in flat space. This resemblance cannot be perfect at finite $N$, however, as the transition between confined and deconfined behavior must be smooth rather than sharp for any system with a finite number of degrees of freedom. In the $N \rightarrow \infty$ limit, on the other hand, the number of degrees of freedom is infinite even at finite volume, and the deconfinement transition mimics its

\footnotetext{
${ }^{19} \mathrm{~A}$ notable exception is the case of two dimensional gauge theories, which one can explicitly solve in the large $N$ limit in many cases (see, for instance, [83, 54]). In this limit one obtains a Hagedorn-like spectrum with Regge trajectories. Moreover, there is also a stringy description of two dimensional QCD [39].

${ }^{20}$ The AdS/CFT correspondence, which establishes a duality between a gauge theory on a sphere and a string theory, motivates this study.
} 
flat space counterpart more closely; in particular it remains sharp.

Note that the Polyakov loop is no longer a good order parameter in the finite volume theory because its expectation value vanishes for kinematical reasons (Gauss' law makes it impossible to put a single fundamental quark on a compact manifold, independently of the phase the theory is in). However, as we will explain in $\S 5.7$, it is possible to define related order parameters, either by looking at the norm of the Polyakov loop or by introducing an infinitesimal amount of fundamental matter. Alternately, one can use the second order parameter discussed in $\S 2.2$ above; the high and low temperature phases are sharply distinguished by the fact that the free energy scales as $\mathcal{O}\left(N^{2}\right)$ and $\mathcal{O}(1)$, respectively, in these phases. It is still reasonable to write the effective Landau-Ginzburg free energy for this theory as the theory of a complex scalar field $(\mathcal{P})$ on the compact space, and we will use a variant of this description in our analysis later in this paper.

We have argued above that a large $N$ confining gauge theory, compactified on a space of size $R \gg 1 / \Lambda_{Q C D}$, undergoes a phase transition at a temperature of order $\Lambda_{Q C D}$. In this paper we will study the opposite limit $R \Lambda_{Q C D} \ll 1$. The gauge theory is weakly coupled in this limit (recall that the gauge coupling stops running at scale $\frac{1}{R}$ on a compact space), and the thermodynamics may reliably be computed at all values of the temperature. We will find that the phase transition persists in this limit at a temperature of order $\mathcal{O}\left(\frac{1}{R}\right)$. In the case of the $d=4 \mathcal{N}=4$ SYM theory on $S^{3}$, the AdS/CFT correspondence has established [86, 48] that, at strong coupling $\lambda$, the theory undergoes a phase transition at a temperature of $\mathcal{O}\left(\frac{1}{R}\right)$. We will establish that this phase transition continues to occur at weak coupling $\lambda$ at a temperature of the same order.

The weak coupling nature of the phase transitions we describe in this paper (at $R \Lambda_{Q C D} \ll 1$ for confining theories, and at $\lambda \ll 1$ for the $\mathcal{N}=4$ SYM theory) allows us to analyze them in detail. Quite remarkably we will find sharp signatures of stringy thermodynamics in these theories. In particular, we will demonstrate that the low temperature phase is always characterized by a Hagedorn growth in the density of states (see also [77, 73]). We view this result as evidence for the existence of string duals for weakly coupled gauge theories on compact manifolds. 


\section{Partition function for free Yang-Mills theory on a compact space}

We now proceed to directly analyze the thermodynamics of weakly coupled Yang-Mills theory on a compact space, beginning in this section (and the next two) with the free theory. For simplicity, we restrict here to spaces (such as $S^{d-1}$ ) for which all modes of the various fields are massive. In most of this section we will discuss the specific case of a $U(N)$ gauge theory with fields in the adjoint representation, but it is easy to generalize our arguments to more general theories. In particular, our exact results for the partition function in $\S 3.3$ will be completely general. As usual, the behavior of thermodynamic quantities in the canonical ensemble is governed by the partition function

$$
Z(\beta)=\sum_{\text {physical states }} x^{E_{i}}=\int \rho(E) x^{E} d E,
$$

where $\rho(E)$ is the density of states and we define $x \equiv e^{-\beta}=e^{-1 / T}$. As we discussed earlier, it is essential to keep in mind that the constraint from Gauss' law for a gauge theory on a compact space implies that the physical states over which we sum must be gauge-invariant.

\subsection{Two matrix harmonic oscillators give Hagedorn behavior for large $N$}

By expanding the fields into modes on the compact space (e.g., spherical harmonics on $S^{3}$ ), our field theory may be viewed as a quantum mechanical system with infinitely many degrees of freedom (one for each mode). In the free theory, these degrees of freedom are decoupled harmonic oscillators, each in some representation of the gauge group. Before embarking on our detailed analysis of this theory, we present a simple demonstration that even restricting to two such oscillators in the adjoint representation of $U(N)$ yields a theory that displays Hagedorn-like behavior in the large $N \operatorname{limit}^{21}$.

Thus, we consider two such modes, each with unit energy, created by operators $A^{\dagger}$ and $B^{\dagger}$ in the adjoint of $U(N)$. Physical states are gaugeinvariant and correspond to traces of products of these operators acting on the Fock space vacuum. Single-trace states of energy $E$ are specified by a series of $E A^{\dagger}$ 's and $B^{\dagger}$ 's inside the trace. The number of such states satisfies

$$
2^{E} / E<n(E) \leq 2^{E},
$$

\footnotetext{
${ }^{21}$ Hagedorn-like behavior in free large $N$ systems was also observed in [47].
} 
where the upper bound arises since for each of the $E$ positions in the trace we may choose $A^{\dagger}$ or $B^{\dagger}$, and the lower bound comes because cyclicity of the trace equates a given state to at most $E-1$ others. In the large $N$ theory all of these states are independent, so the density of independent single-trace states is Hagedorn-like:

$$
\rho(E)=\frac{\partial n}{\partial E} \sim E^{\alpha} e^{\beta_{H} E},
$$

where the Hagedorn temperature in this case is $T_{H}=1 / \beta_{H}=1 / \ln (2)$.

For finite $N$, we cannot have Hagedorn behavior at all energies, since the high temperature behavior must be that of a field theory (or quantum mechanics in the toy example described above) with finitely many fields. The departure from Hagedorn behavior at high energies comes about because the oscillators have only finitely many gauge-invariant degrees of freedom (of order $N^{2}$ ), so that only a number of traces of order $N^{2}$ give independent states $^{22}$. Thus, at energies of order $N^{2}$, trace relations will cut off the exponential growth of states, resulting in a cross-over to field theory (or quantum mechanics) behavior. In the limit of large $N$, this cross-over becomes a sharp transition, as we will see explicitly in the next sections.

\subsection{Exact partition function for $N=\infty$}

We now show that the exact partition function of our free gauge theory in the strict $N=\infty$ limit (where no trace relations exist) may be obtained by simple counting arguments, following [77, 73].

We consider a model with $m \geq 2$ matrix-valued bosonic harmonic oscillators; the number $m$ can be finite or infinite, as in the example of a compactified $d$-dimensional gauge theory. Let the $i^{\text {th }}$ oscillator have energy $E_{i}$. We encode the spectrum of oscillators in a "single-particle partition function" $z(x) \equiv \sum_{i} x^{E_{i}}$ where the sum goes over all oscillators ${ }^{23}$. Examples of such single-particle partition functions for compactified $3+1$ dimensional theories will be provided in $\S 3.5$ below.

Following the discussion above, the partition function of single-trace states with $k$ oscillators is given by

$$
Z_{k}=\frac{z(x)^{k}}{k}+(\text { positive })
$$

\footnotetext{
${ }^{22}$ For example, for a single $N \times N$ matrix, $\operatorname{tr}\left(\mathrm{A}^{\mathrm{N}+1}\right)$ may be expressed as a combination of products of traces of lower powers of $A$.

${ }^{23}$ To be precise, this is the single-particle partition function for the $U(1)$ theory, since in general the single oscillator states are non-physical.
} 
where the factor of $1 / k$ compensates for cyclicity in the trace, and the + (positive) acknowledges that this is an over-compensation in cases where the trace breaks up into repeated sequences of oscillators. Since in the large $N$ limit a single-trace state can have any number of oscillators, we need to sum this result over $k$ to find a partition function for single-trace states,

$$
Z_{S T}=\sum_{k=1}^{\infty} Z_{k}=-\ln (1-z(x))+\text { (positive). }
$$

Actually, it is not too difficult to promote (3.5) to an exact expression which correctly accounts for repetitions inside the trace. This careful counting utilizes Polya theory and was already performed in [77, 73]. For the convenience of the reader we reproduce it in appendix $\mathrm{B}$. The exact result in the large $N$ limit is

$$
Z_{S T}=-\sum_{q=1}^{\infty} \frac{\varphi(q)}{q} \ln \left(1-z\left(x^{q}\right)\right),
$$

where $\varphi(q)$ represents the number of positive integers which are not larger than $q$ and are relatively prime to $q$. Note that the first term in (3.6) is precisely the term we explicitly wrote down in (3.5).

Using (3.6), we may now write the full partition function of our model, summing over states with arbitrarily many traces. The space of multi-trace states is simply the Fock space of single-trace states, so the full multi-trace partition function $Z$ is easily obtained from $Z_{S T}$ (taking into account the fact that the single-trace states behave as identical bosonic particles),

$$
\begin{aligned}
\ln (Z) & =\sum_{n=1}^{\infty} \frac{1}{n} Z_{S T}\left(x^{n}\right) \\
& =-\sum_{n=1}^{\infty} \frac{1}{n} \sum_{q=1}^{\infty} \frac{\varphi(q)}{q} \ln \left(1-z\left(x^{q n}\right)\right) \\
& =-\sum_{k=1}^{\infty} \frac{1}{k}\left(\sum_{q \mid k} \varphi(q)\right) \ln \left(1-z\left(x^{k}\right)\right) \\
& =-\sum_{k=1}^{\infty} \ln \left(1-z\left(x^{k}\right)\right),
\end{aligned}
$$

where we have used the formula $\sum_{q \mid k} \varphi(q)=k$. It is easy to generalize this result to include also fermionic oscillators; denoting by $z_{B}$ the single-particle partition function of the bosonic oscillators, and by $z_{F}$ the single-particle partition function of the fermionic oscillators, we obtain

$$
\ln (Z)=-\sum_{k=1}^{\infty} \ln \left(1-z_{B}\left(x^{k}\right)+(-1)^{k} z_{F}\left(x^{k}\right)\right) .
$$


The inclusion of fermions does not change the qualitative behavior, so it is sufficient to assume that all oscillators are bosonic for the purposes of qualitative discussions.

Equation (3.8) gives the exact partition function in the strict $N=\infty$ theory. It is not correct for the finite $N$ theory because we have assumed all traces to be independent. ${ }^{24}$ However, as long as the thermodynamics is dominated by states for which the number of oscillators in a trace is small compared to $N^{2}$ (which will certainly be true for low enough temperatures), trace relations will be unimportant and (3.8) should give a very good approximation to the correct behavior.

In order to understand the dynamics of (3.7) we note some obvious properties of the "single-particle partition function" $z(x)$, namely that $z(0)=0$, $z(x)$ is a monotonically increasing function of $x$, and that (since $m \geq 2$ ) $z(1)>1$. It follows that the equation $z(x)=1$ has a unique solution (which we will denote by $x=x_{H}$ ) for $0<x<1$. If we denote the corresponding inverse temperature by $\beta_{H}\left(e^{-\beta_{H}}=x_{H}\right)$, then $\ln (Z(\beta))$ is well defined for $\beta>\beta_{H}$, but diverges like $-\ln \left(\beta-\beta_{H}\right)$ as $\beta$ tends to $\beta_{H}$ from above. For $\beta<\beta_{H}$ (high temperatures) $Z(\beta)$ in (3.7) is ill defined. All these features are reproduced by the formula ${ }^{25}$

$$
Z(\beta)=\int d E \rho(E) e^{-\beta E}
$$

where $\rho(E) \approx e^{\beta_{H} E}$ at high energies. Consequently, our system of oscillators has Hagedorn-like thermodynamics with the inverse Hagedorn temperature $\beta_{H}$. Note that $z(x)=2 x$ for the two oscillator model of the previous subsection, yielding $x_{H}=1 / 2$ and $\beta_{H}=\ln (2)$ in agreement with the discussion in that subsection.

The fact that $Z(\beta)$ diverges for $T>T_{H}=\beta_{H}^{-1}$ indicates that $T_{H}$ is a limiting temperature if $N$ is set strictly equal to infinity. Of course, this cannot be correct at any finite $N$, no matter how large. Indeed, a system of $m N^{2}$ harmonic oscillators (or even a compactified field theory for which $m$

\footnotetext{
${ }^{24}$ When the number of fields in a trace is of order $N^{2}$ trace identities relate various states that are assumed to be different in (3.7). For example, any $2 \times 2$ Hermitian matrix $M$ obeys $\operatorname{tr}\left(\mathrm{M}^{3}\right)=\frac{1}{2}\left(3 \operatorname{tr}(\mathrm{M}) \operatorname{tr}\left(\mathrm{M}^{2}\right)-\operatorname{tr}(\mathrm{M})^{3}\right)$.

${ }^{25}$ Let$$
\ln (Z)=\int d E \rho^{\prime}(E) e^{-\beta E}
$$

and

$$
Z_{S T}=\int d E \rho_{S T}(E) e^{-\beta E}
$$

Then, $\rho^{\prime}(E) \sim \rho_{S T}(E) \sim e^{\beta_{H} E} / E$ at high energies. Note that (3.9) and (3.10) match (2.2) and (2.1), respectively, upon setting $d=d_{e f f}=1$ in those formulas.
} 
is infinite but there is a finite number of oscillators below any fixed energy) can certainly be heated to any temperature. It must instead be the case that at high temperatures $\left(T>T_{H}\right)$, the condition for the validity of (3.8) (namely, that the number of fields contained in the typical state is much smaller than $N^{2}$ ) fails; we will see in $\S 5$ that this is indeed the case. Thus, in the high temperature phase, trace relations invalidate the analysis above, and traces no longer provide a useful basis for $U(N)$ singlets in the high temperature phase. This is true at any finite $N$ and so it is also true in the large $N$ limit of principal interest to us. In the next subsection we therefore turn to a more generally applicable method of dealing with the $U(N)$ singlet condition.

\subsection{Exact partition function for free Yang-Mills theory}

In this section, we will derive an exact expression for the partition function of free Yang-Mills theory with arbitrary gauge group and matter content, on any compact space for which all modes of the various fields are massive. As we have discussed, the basic excitations of such a theory are singleparticle states, corresponding to oscillators which arise by expanding the various fields into physical modes on the space. The general physical state is obtained by acting with arbitrary collections of these oscillators on the Fock space vacuum, with the constraint that the total state should be a singlet of the gauge group.

To obtain an explicit expression for the partition function, suppose we have bosonic modes with energies $E_{i}$ in representations $R_{i}$ of the gauge group and fermionic modes with energies $E_{i}^{\prime}$ in representations $R_{i}^{\prime}$ of the gauge group. ${ }^{26}$ Then, the partition function may be expressed as a sum over the occupation numbers of all modes, with a Boltzmann factor $e^{-\beta E}$ corresponding to the total energy, and a numerical factor counting the number of singlets (physically allowed states) in the corresponding product of representations,

$$
\begin{aligned}
Z(x)= & \sum_{n_{1}=0}^{\infty} x^{n_{1} E_{1}} \sum_{n_{2}=0}^{\infty} x^{n_{2} E_{2}} \cdots \sum_{n_{1}^{\prime}=0}^{\infty} x^{n_{1}^{\prime} E_{1}^{\prime}} \sum_{n_{2}^{\prime}=0}^{\infty} x^{n_{2}^{\prime} E_{2}^{\prime}} \cdots \times \\
& \left\{\# \text { of singlets in } \operatorname{sym}^{n_{1}}\left(R_{1}\right) \otimes \operatorname{sym}^{n_{2}}\left(R_{2}\right) \otimes \cdots\right. \\
& \left.\otimes \operatorname{anti}^{n_{1}^{\prime}}\left(R_{1}^{\prime}\right) \otimes \operatorname{anti}^{n_{2}^{\prime}}\left(R_{2}^{\prime}\right) \otimes \cdots\right\} .
\end{aligned}
$$

\footnotetext{
${ }^{26}$ Prior to this subsection we have assumed that all modes are in the adjoint representation. We relax that assumption in this subsection.
} 
Note that in order to correctly account for particle statistics, we must symmetrize (antisymmetrize) representations corresponding to identical bosonic (fermionic) modes. An explicit expression for the group theory factor may be obtained by using the fact that the number of singlets in a product of representations is simply the integral over the group manifold of the product of characters for these representations, as reviewed in appendix A. Thus, we find

$$
\begin{gathered}
Z(x)=\int[d U] \prod_{i}\left\{\sum_{n_{i}=0}^{\infty} x^{n_{i} E_{i}} \chi_{\operatorname{sym}^{n_{i}}\left(R_{i}\right)}(U)\right\} \\
\cdot \prod_{i}\left\{\sum_{n_{i}^{\prime}=0}^{\infty} x^{n_{i}^{\prime} E_{i}^{\prime}} \chi_{\operatorname{anti}^{n_{i}^{\prime}}\left(R_{i}^{\prime}\right)}(U)\right\} .
\end{gathered}
$$

The sums in brackets are generating functions for the characters of symmetrized (antisymmetrized) products of arbitrarily many copies of a given representation $R_{i}\left(R_{i}^{\prime}\right)$. As we show in appendix $\mathrm{A}$, these turn out to have simple expressions given by equation (A.8). Using this equation, we find that

$$
\begin{aligned}
Z(x)=\int[d U] \exp \left\{\sum_{i ; m=1}^{\infty} \frac{1}{m} x^{m E_{i}} \chi_{R_{i}}\left(U^{m}\right)\right\} \\
\cdot \exp \left\{\sum_{i ; m=1}^{\infty} \frac{(-1)^{m+1}}{m} x^{m E_{i}^{\prime}} \chi_{R_{i}^{\prime}}\left(U^{m}\right)\right\} .
\end{aligned}
$$

Finally, since the sums over $i$ in the exponentials are simply sums over the single-particle modes, it is convenient to define bosonic and fermionic singleparticle partition functions for each representation $R$, counting the singleparticle states in this representation without the degeneracy coming from the dimension of the representation and without any gauge-invariance constraints,

$$
z_{B}^{R}(x)=\sum_{R_{i}=R} x^{E_{i}}, \quad z_{F}^{R}(x)=\sum_{R_{i}^{\prime}=R} x^{E_{i}^{\prime}}
$$

With these definitions, we may express our final result for the partition function as [77]

$$
Z(x)=\int[d U] \exp \left\{\sum_{R} \sum_{m=1}^{\infty} \frac{1}{m}\left[z_{B}^{R}\left(x^{m}\right)+(-1)^{m+1} z_{F}^{R}\left(x^{m}\right)\right] \chi_{R}\left(U^{m}\right)\right\} .
$$

Thus, the partition function for an arbitrary free field theory (with no zero modes) on a compact space may be expressed as a single-matrix integral, 
with the action determined in a simple way by the single-particle modes of the theory.

\subsection{Generalization to arbitrary chemical potential}

Before considering specific gauge theories, we note that the result (3.16) may be easily generalized to the case of a non-zero chemical potential. For a set of commuting charges $Q^{a}$ which commute with the Hamiltonian, we may introduce chemical potentials $\mu_{a} \equiv \ln \left(q_{a}\right)$ simply by replacing $x^{E_{i}} \rightarrow$ $x^{E_{i}} \prod_{a} q_{a}^{Q_{i}^{a}}$ in (3.12), where $Q_{i}^{a}$ is the $a^{t h}$ charge of the $i^{t h}$ mode. The resulting partition function is

$$
\begin{aligned}
Z\left(x,\left\{q_{a}\right\}\right)=\int[d U] \exp \left\{\sum_{R}\right. & \sum_{m=1}^{\infty} \frac{1}{m}\left[z_{B}^{R}\left(x^{m},\left\{q_{a}^{m}\right\}\right)\right. \\
& \left.\left.+(-1)^{m+1} z_{F}^{R}\left(x^{m},\left\{q_{a}^{m}\right\}\right)\right] \chi_{R}\left(U^{m}\right)\right\},
\end{aligned}
$$

where the generalized single-particle partition functions are now given by

$$
z^{R}\left(x,\left\{q_{a}\right\}\right)=\sum_{R_{i}=R} x^{E_{i}} \prod_{a} q_{a}^{Q_{i}^{a}} .
$$

\section{5 $U(N)$ gauge theories with adjoint matter on $S^{3} \times \mathbb{R}$}

For most of the remainder of this paper, we will focus on $U(N)$ gauge theories on a sphere with adjoint matter. In this case, using $\chi_{a d j}(U)=\operatorname{tr}(\mathrm{U}) \operatorname{tr}\left(\mathrm{U}^{\dagger}\right)$ (where we denote by $\operatorname{tr}$ without a subscript the trace in the fundamental representation), we obtain the unitary matrix model

$$
Z(x)=\int[d U] \exp \left\{\sum_{m=1}^{\infty} \frac{1}{m}\left(z_{B}\left(x^{m}\right)+(-1)^{m+1} z_{F}\left(x^{m}\right)\right) \operatorname{tr}\left(\mathrm{U}^{\mathrm{m}}\right) \operatorname{tr}\left(\left(\mathrm{U}^{\dagger}\right)^{\mathrm{m}}\right)\right\} .
$$

It is straightforward to work out explicit expressions for the single particle partition functions for various types of fields on a sphere of unit radius. In appendix B (see also $[77,73])$ we find that for $(3+1)$-dimensional scalars, vectors, and chiral fermions on $S^{3} \times \mathbb{R}$,

$$
z_{S 4}(x)=\frac{x+x^{2}}{(1-x)^{3}}, \quad z_{V 4}(x)=\frac{6 x^{2}-2 x^{3}}{(1-x)^{3}}, \quad z_{F 4}(x)=\frac{4 x^{\frac{3}{2}}}{(1-x)^{3}} .
$$

In a $3+1$ dimensional free $U(N)$ gauge theory with $n_{S}$ scalar fields, $n_{V}$ vector fields and $n_{F}$ chiral fermions (all in the adjoint representation), we should 
use in (3.19) $z_{B}(x)=n_{S} z_{S 4}(x)+n_{V} z_{V 4}(x), z_{F}(x)=n_{F} z_{F 4}(x)$. Note that all of these functions (and the single-particle partition functions in general) increase monotonically from $z=0$ at $x=0$ (zero temperature), and in all dimensions above $0+1$ they diverge at $x=1$ (infinite temperature) ${ }^{27}$.

For $S U(N)$ gauge theories, the only difference (beyond the fact that $U$ must have determinant one) is that every $\operatorname{tr}(\mathrm{U}) \operatorname{tr}\left(\mathrm{U}^{\dagger}\right)$ in (3.19) should be replaced everywhere by $\left(\operatorname{tr}(U) \operatorname{tr}\left(U^{\dagger}\right)-1\right)$, the character of the adjoint representation in $S U(N)$.

\section{Path integral derivation of the matrix integral and an order parameter}

In the previous section we computed the partition function of free Yang-Mills theory on a compact space $\mathcal{M}$ by counting gauge-invariant states. However, $Z(T)$ may also be computed by the vacuum path integral of Euclidean YangMills theory ${ }^{28}$ on $\mathcal{M} \times S_{T}^{1}$ where the circumference of the thermal circle $S_{T}^{1}$ is $\beta=1 / T$. In this section we will rederive the expression (3.19) for the partition function by computing this path integral for gauge theories on an $S^{3}$ of unit radius.

Though it gives an identical final result for the partition function, the path integral derivation provides a physical interpretation for the unitary matrix $U$ as the Wilson loop of the gauge field (averaged over the compact space) around the thermal circle. Consequently, $\frac{1}{N} \operatorname{tr}(\mathrm{U})$ is precisely the Polyakov loop operator described in $\S 2.2$; a standard order parameter for deconfinement. We will see in the next section that $\frac{1}{N} \operatorname{tr}(\mathrm{U})$ is also the natural order parameter for a large $N$ phase transition of the matrix model (3.19).

A further advantage of the path integral derivation is that it generalizes easily to the calculation of the large $N$ partition function $Z(T, \lambda)$ at weak 't Hooft coupling $\lambda \equiv g_{Y M}^{2} N$ when the theory is no longer free. In $\S 6.2$ we analyze the general structure of $Z(T, \lambda)$ at small $\lambda$. An explicit calculation of the weak-coupling partition function, in specific gauge theories, is postponed to a future paper [3].

\footnotetext{
${ }^{27}$ For a $d$ dimensional field theory compactified on $S^{d-1}, z(x)$ diverges as $2 \mathcal{N}^{\text {dof }} /(1-$ $x)^{d-1}$, where $\mathcal{N}^{\text {dof }}$ is the number of single-particle degrees of freedom.

${ }^{28}$ We thank Nima Arkani Hamed for emphasizing the utility of this approach to us, and for several extremely enjoyable and productive conversations on related issues.
} 


\subsection{Basic set-up}

We work in the gauge

$$
\partial_{i} A^{i}=0
$$

where $i=1,2,3$ runs over the sphere coordinates, and $\partial_{i}$ are covariant derivatives. The choice (4.1) fixes the gauge freedom only partially; it leaves spatially independent but time dependent gauge transformations unfixed. We fix this residual gauge-invariance with the condition

$$
\partial_{t} \alpha(t)=0
$$

where

$$
\alpha \equiv \frac{1}{\omega_{3}} \int_{S^{3}} A_{0},
$$

where $\omega_{3}$ is the volume of $S^{3}$.

The mode $\alpha$ will play a special role in what follows because it is the only zero mode (mode whose action vanishes at quadratic order) in the decomposition of Yang-Mills theory into Kaluza-Klein modes on $S^{3} \times S^{1}$. Consequently, $\alpha$ fluctuations are strongly coupled at every value of $\lambda$, including in the limit $\lambda \rightarrow 0$. In particular, they cannot directly be integrated out in perturbation theory.

In order to proceed with the perturbative evaluation of the partition function we will adopt a two step procedure. In the first step, discussed in this section, we integrate out all non-zero modes ${ }^{29}$ and generate an effective action $S_{\text {eff }}(\alpha)$ for $\alpha$. $S_{\text {eff }}(\alpha)$ is non-trivial even at zero coupling and, as we will discuss in $\S 6$, it is further modified perturbatively in $\lambda$. Once we have obtained $S_{\text {eff }}(\alpha)$, we must then proceed to perform the integral over $\alpha$ in order to obtain $Z(T)$. This is the subject matter of $\S 5$.

\subsection{The integration measure}

In this subsection we will define $S_{\text {eff }}(\alpha)$ more carefully. Recall that the Yang-Mills free energy may be written as

$$
e^{-\beta F}=\int d \alpha \int \mathcal{D} A \Delta_{1} \Delta_{2} e^{-S_{Y M}(A, \alpha)},
$$

where $\Delta_{1}$ is the Fadeev Popov determinant conjugate to (4.1), $\Delta_{2}$ is the Fadeev Popov determinant conjugate to (4.2), and $S_{Y M}$ is the Yang-Mills

\footnotetext{
${ }^{29}$ As $\alpha$ is a zero mode this is a standard Wilsonian procedure. Note the parallel with the discussions in $\S 2.2$ and in appendix $\mathrm{C}$.
} 
action. It is not difficult to explicitly evaluate $\Delta_{2}$ (see later in this subsection) and verify that it is independent of $A$. Consequently, (4.4) may be rewritten as

$$
e^{-\beta F}=\int d \alpha \Delta_{2} \exp \left[-S_{e f f}(\alpha)\right]
$$

where

$$
\exp \left[-S_{e f f}(\alpha)\right]=\int \mathcal{D} A \Delta_{1} \exp \left[-S_{Y M}(A, \alpha)\right] .
$$

In the rest of this subsection, we will explicitly evaluate $\Delta_{2}$ and so determine the effective measure of integration (4.5).

It follows from (4.2) that

$$
\Delta_{2}=\operatorname{det}^{\prime}\left(\partial_{0}-i[\alpha, *]\right),
$$

where the prime asserts that the determinant is over non-zero modes of $A_{0}$. Denoting by $\lambda_{i}(i=1, \cdots, n)$ the eigenvalues of $\alpha$, and choosing a convenient basis of matrix functions whose time-dependence is given by $\exp (2 \pi i n t / \beta)$, the determinant is easily evaluated as the product

$$
\begin{aligned}
\Delta_{2} & =\prod_{n \neq 0} \prod_{i, j}\left[\frac{2 \pi i n}{\beta}-i\left(\lambda_{i}-\lambda_{j}\right)\right] \\
& =\left(\prod_{m \neq 0} \frac{2 \pi i m}{\beta}\right) \prod_{i, j} \frac{2}{\beta\left(\lambda_{i}-\lambda_{j}\right)} \sin \left(\frac{\beta\left(\lambda_{i}-\lambda_{j}\right)}{2}\right) .
\end{aligned}
$$

Notice that up to an overall constant,

$$
d \alpha \Delta_{2}=[d U]
$$

where

$$
d \alpha=\prod_{i} d \lambda_{i} \prod_{i<j}\left(\lambda_{i}-\lambda_{j}\right)^{2}
$$

is the left-right invariant integration measure over Hermitian matrices $\alpha$, and

$$
[d U]=\prod_{i} d \lambda_{i} \prod_{i<j} \sin ^{2}\left(\frac{\beta\left(\lambda_{i}-\lambda_{j}\right)}{2}\right)
$$

is the left-right invariant integration measure in the integral over the unitary matrices

$$
U \equiv e^{i \beta \alpha}
$$

We will see in the next section at one loop order and argue generally in $\S 6$ that $S_{\text {eff }}$ may be regarded as a function of $U$ rather than $\alpha$, so that (4.5) may be written as

$$
e^{-\beta F}=\int[d U] e^{-S_{e f f}(U)}
$$

where $S_{e f f}(U)$ is defined in (4.6). 


\subsection{Evaluation of $S_{\text {eff }}$ at one-loop}

The path integral in (4.5) may be evaluated diagrammatically, generating an expansion of $S_{\text {eff }}(U)$ in powers of the gauge coupling. In this subsection we use this method to evaluate $S_{\text {eff }}(U)$ to lowest order in $\lambda\left(\mathcal{O}\left(\lambda^{0}\right)\right)$. This evaluation is rather simple as only one-loop graphs in the expansion of (4.5) contribute to $S_{\text {eff }}$ at this order.

We first consider the pure gauge theory. The Fadeev-Popov determinant for gauge-fixing (4.1) is

$$
\operatorname{det} \partial_{i} D^{i}=\int \mathcal{D} c \mathcal{D} \bar{c} e^{-\bar{c} \partial_{i} D^{i} c}
$$

where $D^{i}$ denotes a gauge covariant derivative

$$
D_{i} c=\partial_{i}-i\left[A_{i}, c\right]
$$

and $c$ and $\bar{c}$ are complex ghosts. Consequently, the action of the free gauge theory may be written as

$$
\begin{aligned}
e^{-S_{\text {eff }}(U)}=\int & \mathcal{D} A_{i} \mathcal{D} A_{0} \mathcal{D} c \mathcal{D} \bar{c} \delta\left(\partial_{i} A^{i}\right) \\
& \cdot \exp \left[-\int \operatorname{Tr}\left(\frac{1}{2} \mathrm{~A}_{\mathrm{i}}\left(\widetilde{\mathrm{D}}_{0}^{2}+\partial^{2}\right) \mathrm{A}^{\mathrm{i}}+\frac{1}{2} \mathrm{~A}_{0} \partial^{2} \mathrm{~A}_{0}+\overline{\mathrm{c}} \partial^{2} \mathrm{c}\right)\right]
\end{aligned}
$$

where

$$
\widetilde{D}_{0} X \equiv \partial_{0} X-i[\alpha, X]
$$

To proceed further we note that any vector field on the sphere may be decomposed as

$$
A_{i}=\partial_{i} \varphi+B_{i}
$$

where $\partial_{i} B^{i}=0$. The integral over $\varphi$ in (4.16) is easily performed using the $\delta$ function, yielding $1 / \sqrt{\operatorname{det}^{\prime}\left(\partial_{i} \partial^{i}\right)}$ where the derivatives act on scalar functions on $S^{3}$ and the prime denotes omission of the zero mode. The integral over $A_{0}$ yields the identical factor (the zero mode is $\alpha$ which is not integrated over). The integral over the ghosts, on the other hand, evaluates to $\operatorname{det}^{\prime}\left(\partial_{i} \partial^{i}\right)$. These three factors cancel nicely, so that (4.16) simplifies to

$$
e^{-S_{e f f}(U)}=\int \mathcal{D} B_{i} \exp \left[-\frac{1}{2} \int \operatorname{Tr}\left(\mathrm{B}_{\mathrm{i}}\left(\widetilde{\mathrm{D}}_{0}^{2}+\partial^{2}\right) \mathrm{B}^{\mathrm{i}}\right)\right] .
$$

Thus,

$$
S_{\text {eff }}=\frac{1}{2} \ln \left(\operatorname{det}\left(-\widetilde{D}_{0}^{2}-\partial^{2}\right)\right),
$$


where the operator acts on the space of divergenceless vector functions on the sphere, i.e., the space of vector functions spanned by the vector spherical harmonics. Consequently

$$
S_{e f f}=\frac{1}{2} \sum_{\Delta} n(\Delta) \ln \operatorname{det}\left(-\widetilde{D}_{0}^{2}+\Delta^{2}\right),
$$

where $\Delta^{2}$ are the eigenvalues of the Laplacian acting on vector spherical harmonics on the compact manifold and $n(\Delta)$ is the degeneracy of each eigenvalue. When the compact space is an $S^{3}$ of unit radius we have $\Delta=h+1$ (for integer $h \geq 0$ ) and $n(\Delta)=2 h(h+2)$ (see the counting of vector spherical harmonics in appendix B).

The determinant of $\left(-\widetilde{D}_{0}^{2}+\Delta^{2}\right)$ is easily evaluated by passing to Fourier space in the time direction, yielding the infinite product

$$
\operatorname{det}_{U(N)}\left[\prod_{n=-\infty}^{\infty}\left(\frac{4 \pi^{2} n^{2}}{\beta^{2}}+\frac{4 \pi n}{\beta} \alpha+\alpha^{2}+\Delta^{2}\right)\right] .
$$

The infinite product of matrices may be rewritten as

$$
\begin{aligned}
& \left(\alpha^{2}+\Delta^{2}\right)\left[\prod_{m \neq 0} \frac{\beta}{2 \pi m}\right]^{-2}\left[\prod_{n \neq 0}\left(1+\frac{\beta \alpha}{\pi n}+\frac{\left(\alpha^{2}+\Delta^{2}\right) \beta^{2}}{4 \pi^{2} n^{2}}\right)\right] \\
& =\left(\alpha^{2}+\Delta^{2}\right)\left[\prod_{m \neq 0} \frac{\beta}{2 \pi m}\right]^{-2}\left[\prod_{n=1}^{\infty}\left(1-\frac{\beta^{2}(\alpha+i \Delta)^{2}}{4 \pi^{2} n^{2}}\right)\left(1-\frac{\beta^{2}(\alpha-i \Delta)^{2}}{4 \pi^{2} n^{2}}\right)\right] \\
& =\left[\prod_{m \neq 0} \frac{\beta}{2 \pi m}\right]^{-2}\left(\frac{4}{\beta^{2}}\right) \sin \left[\frac{\beta(\alpha+i \Delta)}{2}\right] \sin \left[\frac{\beta(\alpha-i \Delta)}{2}\right] \\
& =\frac{2}{\beta^{2}}\left[\prod_{m \neq 0} \frac{\beta}{2 \pi m}\right]^{-2}[\cosh (\beta \Delta)-\cos (\beta \alpha)] \\
& =\mathcal{N} e^{\beta \Delta}\left(1-e^{-\beta \Delta+i \beta \alpha}\right)\left(1-e^{-\beta \Delta-i \beta \alpha}\right),
\end{aligned}
$$

where

$$
\mathcal{N}=\left(\frac{1}{\beta^{2}}\left[\prod_{m \neq 0} \frac{\beta}{2 \pi m}\right]^{-2}\right)
$$

The divergent factor $\mathcal{N}$ is a constant independent of both $\Delta$ and $\alpha$, and we set it to unity to reproduce the free energy of the harmonic oscillator. 
Thus

$$
\ln \left(\operatorname{det}\left(-\widetilde{D}_{0}^{2}+\Delta^{2}\right)\right)=\operatorname{Tr}\left(\beta \Delta+\ln \left(1-\mathrm{e}^{-\beta \Delta+\mathrm{i} \beta \alpha}\right)+\ln \left(1-\mathrm{e}^{-\beta \Delta-\mathrm{i} \beta \alpha}\right)\right),
$$

where the trace is over $N^{2} \times N^{2}$ dimensional matrices and $\alpha$ acts in the adjoint representation. Expanding the logarithms in a power series, summing over $\Delta$, and passing from the adjoint to the fundamental $\left(\operatorname{Tr}_{\text {adj }}\left(\mathrm{e}^{\mathrm{in} \beta \alpha}\right) \rightarrow\right.$ $\left.\operatorname{tr}\left(\mathrm{U}^{\mathrm{n}}\right) \operatorname{tr}\left(\mathrm{U}^{-\mathrm{n}}\right)\right)$ and using (4.20) we obtain

$$
S_{e f f}=\frac{1}{2} \beta N^{2} \sum_{\Delta} \Delta n(\Delta)-\sum_{n=1}^{\infty} \frac{z_{V}\left(x^{n}\right)}{n} \operatorname{tr}\left(\mathrm{U}^{\mathrm{n}}\right) \operatorname{tr}\left(\mathrm{U}^{-\mathrm{n}}\right) .
$$

For the case of an $S^{3}$ of unit radius the first term (appropriately regularized) is equal to $\frac{11}{120} \beta N^{2}$, where $\frac{11}{120}$ is the Casimir energy $\sum_{0}^{\infty} h(h+1)(h+2)$ for a vector field on the unit sphere. ${ }^{30}$

It is a simple matter to generalize this calculation to include the contributions from free conformally coupled scalar and spinor fields. When the compact manifold is $S^{3}$, the contribution to $S_{\text {eff }}$ from a single additional scalar field is

$$
\delta S_{\text {eff }}=\frac{1}{2} \ln \left(\operatorname{det}\left(-\widetilde{D}_{0}^{2}-\partial^{2}+1\right)\right),
$$

where the operator acts on scalar fields on $S^{3} \times S^{1}$ and the constant piece of the operator is a consequence of the $\mathcal{R} \varphi^{2}$ term in the Lagrangian for

\footnotetext{
${ }^{30}$ See equation (64) of [7]. This equation, and the other Casimir energies in this subsection, may be justified as follows. On a sphere of radius $R$ we wish to compute $\sum_{m=1}^{\infty}\left(m^{2}-1\right)(m / R)$, where $E=m / R$ is the energy of the mode in question. We regulate this sum by multiplying the summand with a cutoff function $f(E / \Lambda)$, where $f$ is a smooth function such that $f(0)=1, f^{\prime}(0)=0$ and $f(\infty)=0$. We now evaluate the regulated sum using the Euler-MacLaurin formula

$$
\frac{1}{2} F(0)+F(1)+F(2) \ldots=\int_{0}^{\infty} F(x) d x+\zeta(-1) F^{\prime}(0)+\zeta(-3) \frac{F^{\prime \prime \prime}(0)}{3 !}+\cdots
$$

with $F(m)=\left(m^{2}-1\right)(m / R) f\left(\frac{m}{R \Lambda}\right)$, and we find

$$
\Lambda^{4} R^{3} \int_{0}^{\infty} x^{3} f(x)-\Lambda^{2} R \int_{0}^{\infty} x f(x)+\frac{-\zeta(-1)+\zeta(-3)}{R}+\mathcal{O}\left(\frac{1}{\Lambda^{2}}\right) .
$$

In order that the vacuum energy of the theory be zero in flat space (a necessary condition, for instance, for conformal invariance), the two divergent terms above must be cancelled by counterterms; indeed, counterterms of the form $a \Lambda^{4} \int \sqrt{g}$ and $b \Lambda^{2} \int \sqrt{g} \mathcal{R}$ (with suitable values for $a$ and $b$ ) achieve this cancellation. The remaining finite piece of the energy is $11 / 120 R$. Finally, note that we have been careful to choose a 'general coordinate invariant' regulator; one that cuts off modes in a manner that depends only on their proper energy. It would be incorrect, for instance, to regulate the summand above with the smoothing function $f((m-1) / \Lambda R)$; in this regulation scheme theories with the same Lagrangian and same $\Lambda$ but different $R$ are not identical in the UV. See chapter 6 of [15] for an interesting and extensive discussion of related issues.
} 
conformally coupled scalars. As above, this determinant is easily evaluated to yield

$$
S_{e f f}=\frac{1}{240} \beta N^{2}-\sum_{n=1}^{\infty} \frac{z_{S}\left(x^{n}\right)}{n} \operatorname{tr}\left(\mathrm{U}^{\mathrm{n}}\right) \operatorname{tr}\left(\mathrm{U}^{-\mathrm{n}}\right),
$$

where $\frac{1}{240}=\frac{1}{2} \sum_{n=0}^{\infty}(n+1)^{3}$. A Weyl spinor field on $S^{3}$ contributes ${ }^{31}$

$$
\begin{aligned}
S_{e f f} & =-\ln \left(\operatorname{det}\left(-\widetilde{\partial}_{0} \gamma^{0}-\not \partial\right)\right) \\
& =\frac{17}{960} \beta N^{2}-\sum_{n=1}^{\infty} \frac{(-1)^{n+1} z_{F}\left(x^{n}\right)}{n} \operatorname{tr}\left(\mathrm{U}^{\mathrm{n}}\right) \operatorname{tr}\left(\mathrm{U}^{-\mathrm{n}}\right)
\end{aligned}
$$

where $\frac{17}{960}=-\sum_{n=1}^{\infty} n(n+1)\left(n+\frac{1}{2}\right)$.

Thus, we have rederived the expression for the free partition function (3.19), but this time with a physical interpretation for the matrix $U$ as the holonomy around the time circle, and for $\frac{1}{N} \operatorname{tr}(\mathrm{U})$ as a Wilson loop around the time circle, or a Polyakov loop (in the fundamental representation).

\section{Solution of the free Yang-Mills matrix model}

In this section we proceed to directly analyze the unitary matrix model (3.19) for the exact partition function of free $U(N)$ Yang-Mills theory in order to extract the thermodynamic behavior.

We begin by recalling that any unitary matrix model with gauge-invariant action and measure may be rewritten entirely in terms of the eigenvalues of the unitary matrix, which must lie on the unit circle. Denoting these eigenvalues by $\left\{e^{i \alpha_{i}}\right\}$ (with $-\pi<\alpha_{i} \leq \pi$ ), we may rewrite the partition function (3.19) in terms of the eigenvalues by the replacements

$$
\int[d U] \rightarrow \prod_{i} \int_{-\pi}^{\pi}\left[d \alpha_{i}\right] \prod_{i<j} \sin ^{2}\left(\frac{\alpha_{i}-\alpha_{j}}{2}\right) ; \quad \operatorname{tr}\left(\mathrm{U}^{\mathrm{n}}\right) \rightarrow \sum_{\mathrm{j}} \mathrm{e}^{\mathrm{in} \alpha_{\mathrm{j}}}
$$

With only adjoint matter, we find from (3.19) that the effective theory for the eigenvalues is governed entirely by a pairwise potential,

$$
Z(x)=\int\left[d \alpha_{i}\right] e^{-\sum_{i \neq j} V\left(\alpha_{i}-\alpha_{j}\right)}
$$

\footnotetext{
${ }^{31}$ We take the spinor field to be anti-periodic on the $S^{1}$ in order to compute the trace of $e^{-\beta H}$ rather than the trace of $(-1)^{F} e^{-\beta H}$.
} 
where

$$
\begin{aligned}
V(\theta) & =-\ln |\sin (\theta / 2)|-\sum_{n=1}^{\infty} \frac{1}{n}\left[z_{B}\left(x^{n}\right)+(-1)^{n+1} z_{F}\left(x^{n}\right)\right] \cos (n \theta) \\
& =\ln (2)+\sum_{n=1}^{\infty} \frac{1}{n}\left(1-z_{B}\left(x^{n}\right)-(-1)^{n+1} z_{F}\left(x^{n}\right)\right) \cos (n \theta) .
\end{aligned}
$$

In the first line, the first term coming from the measure is a temperatureindependent repulsive potential, while the remaining terms provide an attractive potential which increases from zero to infinite strength as the temperature increases from zero to infinity. ${ }^{32}$ This suggests that at low temperatures, the minimum action configurations will correspond to eigenvalues distributed evenly around the circle, while at high temperatures, the eigenvalues will tend to bunch up.

For finite $N$, the partition function receives contributions from all configurations of eigenvalues and depends smoothly on the temperature. On the other hand, it is well known that in the large $N$ limit, the matrix model partition function is dominated by the minimum action configurations, with the exact leading and subleading terms in the $1 / N$ expansion of the free energy given, respectively, by the minimum of the action and by the Gaussian integral about the minimum action configuration. When this minimum action configuration (or its derivatives) changes abruptly as a function of temperature, we may have a phase transition in the large $N$ limit (see, for example, [41]). We will now see that exactly this behavior occurs and leads to a phase transition for our model.

We begin in $\S 5.1$ by analyzing the low temperature phase. In $\S 5.2$ we analyze the behavior near the phase transition temperature, and in $\S 5.3$ we analyze the high temperature limit. In $\S 5.4$ we write down the solution of the matrix model in the high temperature phase, and in $\S 5.5$ we provide a perturbative expansion of this solution around the phase transition temperature. In $\S 5.6$ we summarize the thermodynamical behavior of the theory. In $\S 5.7$ we discuss how the Polyakov loop may be used as an order parameter at finite volume, and we end in $\S 5.8$ with an application of our results to some interesting $3+1$ dimensional gauge theories.

\footnotetext{
${ }^{32}$ To see that the second term in the potential is always attractive, note that we may rewrite it as

$$
V_{2}(\theta)=\frac{1}{2} \int d E\left\{\rho_{B}(E) \ln \left(1-2 x^{E} \cos (\theta)+x^{2 E}\right)-\rho_{F}(E) \ln \left(1+2 x^{E} \cos (\theta)+x^{2 E}\right)\right\},
$$
}

where $\rho_{B}$ and $\rho_{F}$ give the single-particle density of states for bosonic and fermionic modes. This potential is always attractive since for any value of $E$ and $x$ the integrand is decreasing in the interval $\theta \in[-\pi, 0]$ and increasing in the interval $\theta \in[0, \pi]$. 


\subsection{Low temperature behavior}

Consider first the theory at low temperatures, where the effective potential between the eigenvalues is dominated by the repulsive term. In this case, we expect that the pairwise repulsion drives the eigenvalues to spread uniformly around the circle. In fact, since there is no difference between displacing any individual eigenvalue in the uniform distribution to the left and displacing it to the right, the uniform eigenvalue distribution will always be a stationary point of the action for a pairwise potential. To see when this stationary point is a minimum, it is convenient to introduce an eigenvalue distribution $\rho(\theta)$ proportional to the density of eigenvalues $e^{i \theta}$ of $U$ at the point $\theta$. Note that $\rho$ must be everywhere non-negative, and we may choose its normalization so that

$$
\int_{-\pi}^{\pi} d \theta \rho(\theta)=1
$$

With this definition, the effective action for the eigenvalues becomes

$$
\begin{aligned}
S[\rho(\theta)] & =N^{2} \int d \theta_{1} \int d \theta_{2} \rho\left(\theta_{1}\right) \rho\left(\theta_{2}\right) V\left(\theta_{1}-\theta_{2}\right) \\
& =\frac{N^{2}}{2 \pi} \sum_{n=1}^{\infty}\left|\rho_{n}\right|^{2} V_{n}(T),
\end{aligned}
$$

where in the second line, we have defined $\rho_{n} \equiv \int d \theta \rho(\theta) \cos (n \theta)$ and $V_{n} \equiv$ $\int d \theta V(\theta) \cos (n \theta)$ to be the Fourier modes on the circle of $\rho$ and $V$, respectively, and we assume without loss of generality that the eigenvalue distribution is symmetric around $\theta=0$. From the latter expression, it is clear that the uniform distribution $\left(\rho_{n \geq 1}=0\right)$ will be an absolute minimum of the potential as long as all $V_{n}$ are positive. From (5.3) we see that

$$
V_{n}=\frac{2 \pi}{n}\left(1-z_{B}\left(x^{n}\right)-(-1)^{n+1} z_{F}\left(x^{n}\right)\right),
$$

so the uniform distribution is an absolute minimum if and only if

$$
z_{B}\left(x^{n}\right)+(-1)^{n+1} z_{F}\left(x^{n}\right)<1
$$

for all $n$. But, since the single-particle partition functions are monotonically increasing, and $0 \leq x<1$, the $n=1$ condition is always strongest, and the uniform distribution will be stable for temperatures $T<T_{H}=-1 / \ln \left(x_{H}\right)$ where $x_{H}$ is the solution to

$$
z\left(x_{H}\right) \equiv z_{B}\left(x_{H}\right)+z_{F}\left(x_{H}\right)=1 ;
$$

note that this is precisely the Hagedorn temperature we discussed in $§ 3.2$. As long as we have more than a single quantum-mechanical mode, equation 
(5.9) always has a unique solution with $0<x<1$, so the uniform distribution becomes an unstable extremum beyond some finite temperature $T_{H}$ determined by the single-particle partition function.

For $T<T_{H}$, we may now evaluate the free energy at leading and subleading order in $N$. Since $\operatorname{tr}\left(\mathrm{U}^{\mathrm{n}}\right)$ vanishes for any $n \geq 1$ for the uniform distribution, the classical value for the action (3.19) (and thus the leading $\mathcal{O}\left(N^{2}\right)$ contribution to the free energy) vanishes. The first non-zero contribution to the free energy thus arises from the Gaussian integral around this configuration. From the quadratic action (5.6), (5.7), we see that the leading contribution to the partition function is ${ }^{33}$

$$
Z(x)=\prod_{n=1}^{\infty} \frac{1}{1-z_{B}\left(x^{n}\right)-(-1)^{n+1} z_{F}\left(x^{n}\right)},
$$

where the normalization has been arbitrarily fixed by choosing the vacuum free energy to vanish, $Z(x=0)=1$ (more physically, we could choose $Z(0)$ to account for the Casimir energy of the vacuum, see the previous subsection). This is precisely the same as the result (3.8) that we found above by counting the states with $E \ll N^{2}$; we see that this is indeed the leading large $N$ behavior as long as $T<T_{H}$.

This partition function diverges at $T=T_{H}$, as should be expected, since this is the point where the quadratic action (5.6) develops an unstable direction. The corresponding divergence in the free energy $F=-T \ln (Z)$ goes like

$$
F \rightarrow T_{H} \ln \left(T_{H}-T\right)
$$

as $T$ approaches $T_{H}$ from below. By the general discussion in $\S 2$, we may conclude that this divergence is associated with a Hagedorn density of states,

$$
\rho(E) \propto E^{0} e^{\beta_{H} E},
$$

as we also found by counting states in $\S 3$. Thus, just as for perturbative string theory, we have a Hagedorn divergence which is associated with an unstable mode in the Euclidean path integral; note that the power of the energy is also the same as in string theory at finite volume. In this case, the "tachyon" causing the divergence is the lowest "momentum" fluctuation mode $\rho_{1}$ of the eigenvalues. In the next subsection, we will be able to see explicitly what the endpoint of this tachyon condensation brings as we raise the temperature beyond the Hagedorn temperature.

\footnotetext{
${ }^{33}$ The 'Jacobian' for the 'change of variables' from $\lambda_{i}$ to $\rho_{n}$ appears to be irrelevant at every order in the $1 / N$ expansion, see [35].
} 


\subsection{Behavior near the transition}

At the temperature $T=T_{H}, V_{1}$ vanishes and the mode $\rho_{1}$ (the lowest momentum fluctuation in the eigenvalue distribution) becomes massless. Since the action is quadratic, this corresponds to an exactly flat direction in the potential, and the corresponding family of minimum action configurations are given (up to overall translations of $\theta$ ) by

$$
\rho(\theta)=\frac{1}{2 \pi}(1+t \cos (\theta)), \quad 0 \leq t \leq 1,
$$

which interpolate between the uniform distribution and a sinusoid vanishing at a single point. For $T>T_{H}$, the quadratic form $S$ has negative coefficients, so all minimum action configurations must lie at the boundary of configuration space, at a point where a hyperboloid of constant $S$ lies tangent to this boundary. Since this boundary is provided by the positivity condition $\rho(\theta) \geq 0$, these minimum action eigenvalue distributions necessarily vanish on a subset of the circle.

In the limit of small, positive $\Delta T=T-T_{H}$, the action contour $S=0$ is a cone with opening angle going to zero. The contours of smaller $S$ are hyperboloids inside this cone, so the minimum action configuration lies on the boundary of the configuration space inside the cone. Finally, it is easy to see that the region of allowed $\rho$ 's is convex (if points $\left\{\rho_{n}\right\}$ and $\left\{\rho_{n}^{\prime}\right\}$ are in the region then all points $\left\{t \rho_{n}+(1-t) \rho_{n}^{\prime}\right\}$ are in the region for $\left.0 \leq t \leq 1\right)$. Thus, the boundary region interior to the cone is a simply-connected neighborhood of the $t=1$ configuration in (5.13) whose size goes to zero as $T \rightarrow T_{H}$. We conclude that the minimizing configuration changes continuously from the $t=1$ configuration in (5.13) for $T$ in some interval above $T_{H}$.

The leading behavior of the free energy in this vicinity of $T_{H}$ is then given by evaluating the $V_{1}$ term in the action (5.6) on the $t=1$ configuration in (5.13), since the effects of changing the eigenvalue distribution come in at higher orders.

Thus, the leading result for the minimum action is

$$
S_{T>T_{H}}^{\min }(x)=\frac{N^{2}}{8 \pi}\left(T-T_{H}\right) V_{1}^{\prime}\left(T_{H}\right)+\ldots
$$

from which we may obtain the free energy as $F=T S^{\text {min }}$. The leading behavior near the transition is therefore given by

$$
\lim _{N \rightarrow \infty} \frac{1}{N^{2}} F_{T \rightarrow T_{H}}(T)= \begin{cases}0 & T<T_{H} \\ -\frac{1}{4}\left(T-T_{H}\right) z^{\prime}\left(x_{H}\right) \frac{x_{H}}{T_{H}} & T>T_{H}\end{cases}
$$


so we have a first order phase transition at the Hagedorn temperature. Note also that the behavior is characteristic of a deconfinement transition, since the free energy is $\mathcal{O}(1)$ below the transition and $\mathcal{O}\left(N^{2}\right)$ above the transition. This will be verified in $\S 5.7$ below when we discuss the behavior of a second order parameter for confinement, related to the Polyakov loop.

\subsection{High temperature behavior}

For general values of $T>T_{H}$, we will not be able to write down an exact expression for the free energy, though we describe in the next subsection a formal solution that may be used to evaluate the free energy to arbitrary accuracy. However, the theory simplifies again in the large temperature limit, where the potential becomes strongly attractive ${ }^{34}$. In this case, the action is minimized by a tightly clustered configuration of eigenvalues that approaches a delta function in the limit of infinite temperature. Thus, we should have $\rho_{n}=1$ to leading order, and

$$
S=\frac{N^{2}}{2 \pi} \sum_{n=1}^{\infty} V_{n}(T \gg 1) .
$$

The high temperature limit of the single-particle partition functions depends only on the space-time dimension $d$ and the number of physical polarizations $\mathcal{N}^{\text {dof }}$ of the fields in the theory,

$$
z_{i}(x) \rightarrow 2 \mathcal{N}_{i}^{d o f} T^{d-1}+\mathcal{O}\left(T^{d-2}\right)
$$

Using (5.7), we find that the high temperature limit of the free energy is

$$
F(x \rightarrow 1)=-2 N^{2} T^{d} \zeta(d)\left[\mathcal{N}_{B}^{d o f}+\left(1-\frac{1}{2^{d-1}}\right) \mathcal{N}_{F}^{d o f}\right]+\mathcal{O}\left(T^{d-1}\right)
$$

The limiting free energy density here coincides with that of the flat space theory, which should be expected since in the free field analysis we are dealing with a scale-invariant theory, so taking the dimensionless temperature $T R$ to infinity is equivalent to taking the limit of large volume at fixed temperature (see $[20,55,56]$ among others for the high temperature expansion of the free energy for the $U(1)$ theory).

\footnotetext{
${ }^{34}$ The high temperature regime and its relation to string theory was discussed for uncompactified theories in [69] in a very similar language to the one we are using here, and this discussion was applied to two dimensional QCD in [54].
} 


\subsection{Exact solution for $T>T_{H}$}

Having understood the qualitative features of the $T>T_{H}$ behavior, we now describe an exact solution for the matrix model at arbitrary temperatures above the transition. Just beyond the Hagedorn temperature, we have seen that the eigenvalue distribution becomes sinusoidal, vanishing at $\theta=\pi$ as the lowest momentum fluctuation mode condenses. As the temperature is increased further, the attractive term in the pairwise potential continues to increase in strength, so the eigenvalues will become increasingly bunched together, occupying only a finite interval $I=\left[-\theta_{0}, \theta_{0}\right]$ on the circle (we arbitrarily choose the middle of this interval to be at $\theta=0$ for convenience).

The precise distribution may be determined by the condition that a single additional eigenvalue added in the interval $I$ experiences no net force from the other eigenvalues,

$$
\int_{-\theta_{0}}^{\theta_{0}} V^{\prime}(\alpha-\theta) \rho(\theta) d \theta=0, \quad \alpha \in I .
$$

Substituting in our potential (5.3), we obtain

$$
\int_{-\theta_{0}}^{\theta_{0}} \cot \left(\frac{\alpha-\theta}{2}\right) \rho(\theta) d \theta=2 \sum_{n=1}^{\infty} a_{n} \rho_{n} \sin (n \alpha),
$$

where on the right hand side, $a_{n} \equiv z_{B}\left(x^{n}\right)+(-1)^{n+1} z_{F}\left(x^{n}\right)$, and we have used the fact that the eigenvalue distribution should be symmetric about $\theta=0$ and therefore be orthogonal to $\sin (n \theta)$ for all $n$. As before, $\rho_{n}$ are the moments of the eigenvalue distribution,

$$
\rho_{n}=\int_{-\pi}^{\pi} \rho(\theta) \cos (n \theta) d \theta .
$$

To solve for $\rho(\theta)$, it is convenient treat the moments $\rho_{n}$ as independent variables, and self-consistently solve for $\rho(\theta)$ and $\rho_{n}$ together using the two equations (5.20) and (5.21). Fortunately, the equations (5.20) provide the equilibrium conditions for a matrix model with action

$$
S=N \sum_{n=1}^{\infty} \frac{a_{n} \rho_{n}}{n}\left(\operatorname{tr}\left(\mathrm{U}^{\mathrm{n}}\right)+\operatorname{tr}\left(\mathrm{U}^{\dagger \mathrm{n}}\right)\right)
$$

that has been solved exactly in [50]. The solution for $\rho(\theta)$ is ${ }^{35}$

$$
\rho(\theta)=\frac{1}{\pi} \sqrt{\sin ^{2}\left(\frac{\theta_{0}}{2}\right)-\sin ^{2}\left(\frac{\theta}{2}\right)} \sum_{n=1}^{\infty} Q_{n} \cos \left(\left(n-\frac{1}{2}\right) \theta\right)
$$

\footnotetext{
${ }^{35}$ In the language of [50], the solution we are looking for is of type $A_{1}$.
} 
for $-\theta_{0}<\theta<\theta_{0}$ and $\rho(\theta)=0$ otherwise, where

$$
Q_{n} \equiv 2 \sum_{l=0}^{\infty} a_{n+l} \rho_{n+l} P_{l}\left(\cos \left(\theta_{0}\right)\right)
$$

and the angle $\theta_{0}$ that bounds the eigenvalue distribution is determined by the condition

$$
Q_{1}=Q_{0}+2 .
$$

In the formula (5.24) for $Q_{n}$, the $P_{l}$ are Legendre polynomials, defined by

$$
\sum_{l=0}^{\infty} P_{l}(x) z^{l}=\left(1-2 x z+z^{2}\right)^{-\frac{1}{2}} .
$$

We may now eliminate $\rho(\theta)$ to obtain a linear system of equations for the $\rho_{n}$ from (5.21) and (5.25). To describe these, we define a matrix $R$ and a vector $A$ whose elements are polynomials in $s^{2} \equiv \sin ^{2}\left(\theta_{0} / 2\right)$,

$$
\begin{aligned}
R_{m l} & \equiv a_{l} \sum_{k=1}^{l}\left(B^{m+k-\frac{1}{2}}\left(s^{2}\right)+B^{\left|m-k+\frac{1}{2}\right|}\left(s^{2}\right)\right) P_{l-k}\left(1-2 s^{2}\right), \\
A_{m} & \equiv a_{m}\left(P_{m-1}\left(1-2 s^{2}\right)-P_{m}\left(1-2 s^{2}\right)\right)
\end{aligned}
$$

where

$$
B^{n-\frac{1}{2}}\left(s^{2}\right)=\frac{1}{\pi} \int_{-\theta_{0}}^{\theta_{0}} d \theta \sqrt{\sin ^{2}\left(\frac{\theta_{0}}{2}\right)-\sin ^{2}\left(\frac{\theta}{2}\right)} \cos \left(\left(n-\frac{1}{2}\right) \theta\right)
$$

are polynomials defined by the generating function

$$
\sum_{n=0}^{\infty} B^{n+\frac{1}{2}}(x) z^{n}=\frac{1}{2 z}\left(\sqrt{(1-z)^{2}+4 z x}+z-1\right) .
$$

The determining equations (5.21) and (5.25) for the eigenvalue moments and the angle $\theta_{0}$ are then simply

$$
R \vec{\rho}=\vec{\rho} ; \quad \vec{A} \cdot \vec{\rho}=1 .
$$

Thus, the vector of moments of the eigenvalue distribution must be an eigenvector of the matrix $R$ with eigenvalue 1 , normalized so that its dot product with $A$ is 1 (ensuring that the eigenvalue distribution integrates to 1 ). The condition

$$
\operatorname{det}(R-1)=0
$$

that the matrix $R$ has such an eigenvector determines the angle $\theta_{0}$. Note that while this equation is a complicated function of $\theta_{0}$, it is linear in each of 
the coefficients $a_{n}$, so it is convenient to parameterize the solution in terms of $\left(\sin \left(\theta_{0} / 2\right),\left\{a_{n>1}\right\}\right)$, solving for $a_{1}$ in terms of these variables using (5.31). With (5.31) satisfied, the explicit solution for the unit eigenvector may be given as

$$
\rho=M^{-1} e_{1}
$$

where $M$ is a matrix obtained by replacing the first row of the singular matrix $(1-R)$ with the vector $A$, and $e_{1}=(1,0,0, \ldots)$.

This solution is rather formal for the general case when $R$ is an infinite matrix. However, in the case when $a_{n>k}$ vanishes for some $k$, the matrix $M$ takes the form

$$
M=\left(\begin{array}{cc}
M_{k \times k} & 0 \\
L & 1
\end{array}\right)
$$

so the explicit solution may be obtained by inverting a finite matrix,

$$
\vec{\rho}=\left(\begin{array}{cc}
M_{k \times k}^{-1} & 0 \\
-L M_{k \times k}^{-1} & 1
\end{array}\right) e_{1} .
$$

Note that once we evaluate the moments $\rho_{n \leq k}$, the full eigenvalue distribution is given by (5.23) since $Q_{n>k}=0$ in this case. Similarly, only the upper $k \times k$ submatrix of $R$ contributes to the condition (5.31) determining $\theta_{0}$.

In our model, the coefficients $a_{n}$ die off with $n$ (exponentially for $x \ll$ 1 , and like a power law as $x \rightarrow 1$ ) so we may obtain an arbitrarily good approximation to the exact eigenvalue distribution by truncating such that $a_{n>k}=0$ for sufficiently large $k$. It turns out that even restricting to $k=1$ retains the same qualitative behavior of the model. ${ }^{36}$ In this case, the angle $\theta_{0}$ is determined by

$$
0=\operatorname{det}\left(R_{1 \times 1}-1\right)=a_{1}\left(2 s^{2}-s^{4}\right)-1,
$$

while $\rho_{1}$ is determined by

$$
\rho_{1}=M_{1 \times 1}^{-1} \cdot 1=\left(2 a_{1} s^{2}\right)^{-1} .
$$

The full distribution is then determined from $\rho_{1}$ using (5.23) (noting that $\left.Q_{n>1}=0\right)$. Parameterizing the solution by $s=\sin \left(\theta_{0} / 2\right)$, the final result is

$$
\rho(\theta)=\frac{1}{\pi \sin ^{2}\left(\frac{\theta_{0}}{2}\right)} \sqrt{\sin ^{2}\left(\frac{\theta_{0}}{2}\right)-\sin ^{2}\left(\frac{\theta}{2}\right)} \cos \left(\frac{\theta}{2}\right),
$$

\footnotetext{
${ }^{36}$ In this case, the auxiliary theory (5.22) is exactly the model studied in [41]. The truncation to $k=1$ was also considered in [77].
} 
with $\theta_{0}$ determined in terms of $a_{1}$ by (5.35), or explicitly

$$
\sin ^{2}\left(\frac{\theta_{0}}{2}\right)=1-\sqrt{1-\frac{1}{a_{1}(T)}} .
$$

As $T$ increases from $T_{H}$ to infinity, $a_{1}$ increases from 1 to infinity, so $\theta_{0}$ decreases from $\pi$ to 0 , such that the eigenvalue distribution (5.37) eventually approaches a delta function. Using (5.37), we may evaluate the free energy in the approximation $a_{n>1}=0$ and find that

$$
-\frac{1}{N^{2}} \ln \left(Z_{\text {trunc }}\right)=-\left(\frac{1}{2 s^{2}}+\frac{1}{2} \ln \left(s^{2}\right)-\frac{1}{2}\right) .
$$

Near the transition, we find

$$
\frac{F}{N^{2}}=-\frac{T_{H}}{4}\left(a_{1}-1\right)+\mathcal{O}\left(\left(a_{1}-1\right)^{2}\right)=-\left.\frac{T_{H}}{4}\left(T-T_{H}\right) \frac{\partial a_{1}}{\partial T}\right|_{T=T_{H}}+\mathcal{O}\left(\left(T-T_{H}\right)^{2}\right),
$$

which gives the same leading behavior (5.15), characteristic of a first order transition, as we found in the full model.

\subsection{Perturbative expansion slightly above the Hagedorn temperature}

Using the general solution above, it is also possible to determine explicitly the exact behavior near the transition as a perturbation expansion in $\left(T-T_{H}\right)$. To determine $\theta_{0}$ as a function of temperature, it is convenient to define $\Delta \equiv a_{1}-1$ and $\epsilon \equiv \cos ^{2}\left(\theta_{0} / 2\right)$, and to expand $R=R_{0}+\delta R$ where

$$
R_{0} \equiv \operatorname{diag}\left(a_{1}, a_{2}, a_{3}, \ldots\right),
$$

so that $\delta R$ will be small for small $\left(T-T_{H}\right)$. We may then solve the condition (5.31) perturbatively for $\Delta$ as a function of $\epsilon$ by writing

$$
0=\operatorname{det}\left(1-\left(1-R_{0}\right)^{-1} \delta R\right)
$$

and expanding the determinant out in terms of traces. This expansion in powers of $\delta R$ may further be expanded as a power series in $\epsilon$ and $\Delta$, and finally solved perturbatively for $\Delta$ as a power series in $\epsilon$. Using the fact that

$$
\delta R_{m l}=(-1)^{m+l+1} l^{2} m a_{l} \epsilon^{2}+\mathcal{O}\left(\epsilon^{3}\right),
$$

and that the lowest power of $\Delta$ appearing in $(5.42)$ is $1 / \Delta\left(\right.$ from $\left.\left(1-R_{0}\right)_{11}^{-1}\right)$, it follows that $\Delta$ may be obtained to order $\epsilon^{2 k+1}$ by keeping terms to order 
$\delta R^{k}$ in (5.42). Explicitly, we find that the leading terms are

$$
\Delta=\epsilon^{2}+\epsilon^{4}\left(1-\sum_{n=2}^{\infty} \frac{n^{3} a_{n}}{1-a_{n}}\right)+\mathcal{O}\left(\epsilon^{5}\right) .
$$

When $a_{n>1}=0$, this correctly reduces to the leading orders of the truncated model result (5.38)

$$
\Delta=\sum_{n \geq 1} \epsilon^{2 n}
$$

To find perturbative expressions for the moments of the eigenvalue distribution near the transition, we use the general result (5.32), expanding $M=M_{0}+\delta M$ with

$$
\begin{aligned}
M_{0} & =\left(\begin{array}{cccc}
A_{1} & A_{2} & A_{3} & \cdots \\
& 1-a_{2} & & \\
& & 1-a_{3} & \\
& & & \ddots
\end{array}\right) \\
M_{0}^{-1} & =\left(\begin{array}{cccc}
\frac{1}{A_{1}} & \frac{-A_{2}}{A_{1}\left(1-a_{2}\right)} & \frac{-A_{3}}{A_{1}\left(1-a_{3}\right)} & \cdots \\
& \frac{1}{1-a_{2}} & & \\
& & \frac{1}{1-a_{3}} & \\
& & & \ddots
\end{array}\right),
\end{aligned}
$$

so that the explicit perturbative solution is

$$
\rho=M_{0}^{-1}\left(1+\delta M M_{0}^{-1}\right)^{-1} e_{1}=\left(M_{0}^{-1}-M_{0}^{-1} \delta M M_{0}^{-1}+\ldots\right) e_{1} .
$$

Note that

$$
\delta M_{m l}= \begin{cases}-\delta R_{m l} & m>1, \\ 0 & m=1,\end{cases}
$$

so from (5.43) we see that $\delta M$ starts at order $\epsilon^{2}$. Thus, we may obtain the eigenvalue moments to order $\epsilon^{2 k-1}$ by keeping the first $k$ terms here. Using the first two terms shown and the expansions of $a_{1}$ and $A_{n}$ in powers of $\epsilon$, we find

$$
\begin{aligned}
\rho_{1} & =\left(M_{0}^{-1}\right)_{11}+\sum_{n=2}^{\infty}\left(M_{0}^{-1}\right)_{1 n} \delta R_{n 1}\left(M_{0}^{-1}\right)_{11}+\mathcal{O}\left(\epsilon^{4}\right) \\
& =\frac{1}{2}+\frac{\epsilon}{2}+\frac{\epsilon^{2}}{2} \sum_{n=2}^{\infty} \frac{n a_{n}}{1-a_{n}}+\mathcal{O}\left(\epsilon^{3}\right), \\
\rho_{n>1} & =\left(M_{0}^{-1}\right)_{n n} \delta R_{n 1}\left(M_{0}^{-1}\right)_{11}+\mathcal{O}\left(\epsilon^{4}\right) \\
& =\frac{\epsilon^{2}}{2}(-1)^{n} \frac{n}{1-a_{n}}+\mathcal{O}\left(\epsilon^{3}\right) .
\end{aligned}
$$


The result for $\rho_{n>1}$ seems at first somewhat surprising since it appears that the moments increase without bound as $n$ is increased. However, it turns out that the leading terms (5.43) in the expansion of $\delta R_{n 1}$ in $\epsilon$ provide a good approximation only for $\epsilon \ll 1 / n^{2}$. To obtain an approximation valid for all $n$ at some fixed value of $\epsilon$, we should use the complete expression $\delta R_{n 1}=$ $a_{1}\left(B^{n+\frac{1}{2}}(1-\epsilon)+B^{n-\frac{1}{2}}(1-\epsilon)\right)$, which does fall off for large $n$. Alternately, we note that the perturbative expansion of the difference between the moments of the exact distribution and those of the truncated $k=1$ model is well behaved for large $n$,

$$
\begin{array}{r}
\delta \rho_{1}=\rho_{1}-\rho_{1}\left(a_{n>1}=0\right)=\frac{\epsilon^{2}}{2} \sum_{m=2}^{\infty} \frac{m a_{m}}{1-a_{m}}+\mathcal{O}\left(\epsilon^{3}\right), \\
\delta \rho_{n}=\rho_{n>1}-\rho_{n>1}\left(a_{n>1}=0\right)=\frac{\epsilon^{2}}{2}(-1)^{n} \frac{n a_{n}}{1-a_{n}}+\mathcal{O}\left(\epsilon^{3}\right),
\end{array}
$$

due to the exponential decay of $a_{n}$ for large $n$.

Using the expressions (5.49) for $\rho_{n}$, we may finally evaluate the free energy from (5.6) and (5.7). To avoid subtleties associated with the apparent growth in the moments (5.49) for large $n$, it is again convenient to express the result as a correction to the truncated model free energy. The final result, taking into account all corrections to the truncated model, is

$$
\begin{aligned}
-\frac{1}{N^{2}} \ln (Z) & =\sum_{n=1}^{\infty} \frac{1}{n}\left(1-a_{n}\right)\left(\rho_{n}\left(a_{n>1}=0\right)+\delta \rho_{n}\right)^{2} \\
& =-\frac{1}{N^{2}} \ln \left(Z_{\text {trunc }}\right)+\frac{\epsilon^{4}}{4} \sum_{n=2}^{\infty} \frac{n\left(n^{2}-1\right) a_{n}}{1-a_{n}}+\mathcal{O}\left(\epsilon^{5}\right) \\
& =-\frac{\epsilon^{2}}{4}-\frac{\epsilon^{3}}{3}-\epsilon^{4}\left(\frac{3}{8}-\frac{1}{4} \sum_{n=2}^{\infty} \frac{n\left(n^{2}-1\right) a_{n}}{1-a_{n}}\right)+\mathcal{O}\left(\epsilon^{5}\right)
\end{aligned}
$$

where $Z_{\text {trunc }}$ is the partition function (5.39) of the $k=1$ truncation computed earlier in this section. ${ }^{37}$ If desired, the free energy may be rewritten in terms

${ }^{37}$ While the perturbation expansion about the $k=1$ truncated model in the second line of (5.51) is perfectly well behaved, the naive evaluation of $\ln (Z)$ using (5.49) yields the divergent expression

$$
\begin{aligned}
-\frac{1}{N^{2}} \ln (Z) & =\sum_{n=1}^{\infty} \frac{1}{n}\left(1-a_{n}\right) \rho_{n}^{2} \\
& =-\frac{\epsilon^{2}}{4}-\frac{\epsilon^{3}}{2}+\frac{\epsilon^{4}}{4} \sum_{n=2}^{\infty} n-\frac{\epsilon^{4}}{2}+\frac{\epsilon^{4}}{4} \sum_{n=2}^{\infty} \frac{n\left(n^{2}-1\right) a_{n}}{1-a_{n}}+\cdots
\end{aligned}
$$

The divergence in the third term of (5.52) is unphysical; it is a consequence of the (apparent) linear growth in the moments observed in (5.49). As we noted earlier, this linear 
of $\Delta$ using (5.44), or explicitly in terms of temperature using

$$
\Delta=a_{1}(T)-a_{1}\left(T_{H}\right)=a_{1}^{\prime}\left(T_{H}\right)\left(T-T_{H}\right)+\frac{1}{2} a_{1}^{\prime \prime}\left(T_{H}\right)\left(T-T_{H}\right)^{2}+\cdots
$$

\subsection{Summary of thermodynamic behavior}

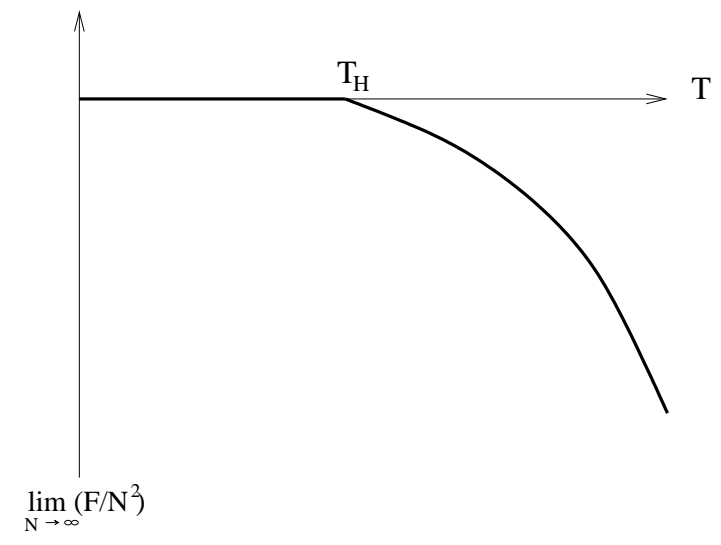

Figure 1: Free energy as a function of temperature in free Yang-Mills theories.

We are now in a position to present a reasonably detailed picture of the behavior of the free energy of the free gauge theory at all temperatures, depicted in figure 1.

We find that the uniform eigenvalue distribution provides an absolute minimum of the matrix model potential for all temperatures below some $T_{H}$ determined by the single-particle partition function as

$$
z\left(T_{H}\right)=1 \text {. }
$$

growth is actually cut off beyond $n \sim 1 / \sqrt{\epsilon}$, where it is replaced by a decaying behavior. Thus, the divergent sum in (5.52) should be cut off at $n=C / \sqrt{\epsilon}$, resulting in a contribution that scales like $C^{2} \epsilon^{3} / 8$; a finite contribution to a lower order of perturbation theory. Indeed, it is easily verified that the (finite) $\epsilon^{3}$ term in (5.52) disagrees with the exact $\epsilon^{3}$ term in the expansion of the solution (5.39)

$$
-\frac{1}{N^{2}} \ln \left(Z_{\text {trunc }}\right)=-\frac{\epsilon^{2}}{4}-\frac{\epsilon^{3}}{3}-\frac{3 \epsilon^{4}}{8}+\mathcal{O}\left(\epsilon^{5}\right),
$$

as our analysis above would suggest. We conclude that the divergent sums at higher orders in the naive perturbation theory conspire to give finite contributions $\epsilon^{3} / 6+\epsilon^{4} / 8+\cdots$. A systematic understanding of this naive perturbation theory would be an intricate task; one that may, however, be avoided by choosing the correct starting point for the perturbation expansion, as we have done above. The smarter perturbation expansion (5.51) is entirely divergence free; this follows since the $a_{n}$ decay exponentially with $n$, whenever $x<1$. 
The free energy in this regime, given by $(5.10)$, is $\mathcal{O}(1)$ and decreases monotonically to a logarithmic divergence $(5.11)$ at $T=T_{H}$, characteristic of a Hagedorn density of states (5.12).

As $T$ increases past $T_{H}$, the uniform eigenvalue distribution develops an unstable mode which condenses to give a pure sinusoid distribution (5.13) for $T$ just above $T_{H}$, and a distribution with a gap for $T>T_{H}$. For small $\left(T-T_{H}\right)$, the $\mathcal{O}\left(N^{2}\right)$ free energy decreases linearly in $T-T_{H}$, so the first derivative of the free energy is discontinuous and we have a first order phase transition. The free energy as a function of temperature may be written as a perturbation expansion in $T-T_{H}$ with the leading terms given in (5.51).

In the high temperature limit, the pairwise potential between eigenvalues becomes strongly attractive, and the eigenvalue distribution approaches a delta function. In this regime, the free energy asymptotes to (5.18) which corresponds to the expected flat-space free-energy density. Using the formal exact solution of the previous section, it should be possible to work out the high temperature behavior as a perturbation expansion in $1 / T$, as we did explicitly for the behavior near the transition.

\subsection{The Polyakov loop as an order parameter at finite volume}

In the theory we analyzed above, the Polyakov loop $\left\langle\frac{1}{N} \operatorname{tr}(\mathrm{U})\right\rangle$ vanishes in both the high temperature and the low temperature phases. However, the reason for this vanishing is rather different in the two phases. The low temperature phase is governed by a single saddle point (uniform distribution of eigenvalues); the Polyakov loop vanishes on this saddle point. On the other hand the high temperature phase is governed by a one parameter set of saddle points (related to each other by the $U(1)$ rotation $U \rightarrow e^{i \alpha} U$, which acts on the eigenvalue distribution by shifting $\theta$ periodically). The value of $\left\langle\frac{1}{N} \operatorname{tr}(\mathrm{U})\right\rangle$ is non-zero when evaluated on each saddle point individually but vanishes upon integrating over $\alpha$ (averaging over all saddle points ${ }^{38}$ ), since the phase varies over a complete circle ${ }^{39}$. To obtain an order parameter which correctly distinguishes the two phases, it is therefore sufficient to use the squared magnitude of the Polyakov loop, $\left\langle|\mathcal{P}|^{2}\right\rangle=\lim _{N \rightarrow \infty}\left\langle\frac{1}{N^{2}}|\operatorname{tr}(\mathrm{U})|^{2}\right\rangle$.

This order parameter may be given a nice physical interpretation by the following alternative definition. Define $\left\langle\frac{1}{N} \operatorname{tr}(\mathrm{U})\right\rangle_{\epsilon}$ as the expectation value

\footnotetext{
${ }^{38}$ Witten [86] has described a rather similar phenomenon in the bulk dual to the strongly coupled $\mathcal{N}=4$ Yang Mills.

${ }^{39}$ For $S U(N)$ theories this is not an integral but a sum over $N$ discrete saddle points, but it has the same effect.
} 
of $\left\langle\frac{1}{N} \operatorname{tr}(\mathrm{U})\right\rangle$ upon perturbing the effective action in (3.19) by the infinitesimal term $N \epsilon\left(\operatorname{tr}(\mathrm{U})+\operatorname{tr}\left(\mathrm{U}^{\dagger}\right)\right)$. Note that this perturbation breaks the $U(1)$ symmetry (or the $\mathbb{Z}_{N}$ symmetry for $S U(N)$ ); in the presence of this perturbation, the high temperature phase is also governed by a single saddle point ${ }^{40}$. Consequently, the order parameter

$$
\mathcal{P}^{e f f}=\lim _{\epsilon \rightarrow 0} \lim _{N \rightarrow \infty}\left\langle\frac{1}{N} \operatorname{tr}(\mathrm{U})\right\rangle_{\epsilon}
$$

is zero in the low temperature phase but non-zero in the high temperature phase $^{41}$. It is easy to show that in the large $N$ limit, $\left\langle|\mathcal{P}|^{2}\right\rangle=\left|\mathcal{P}^{e f f}\right|^{2}$, so the two definitions of the order parameter are equivalent.

The construction presented in the previous paragraph may be understood physically as follows. As discussed in $\S 2.4,\langle\operatorname{tr}(\mathrm{U})\rangle$ vanishes in both phases for purely kinematical reasons; Gauss' law does not allow the introduction of a quark on a compact space. However, the perturbation described in the previous paragraph effectively adds a condensate (or plasma) of classical quarks and anti-quarks to the theory; in the presence of such a condensate the constraint from Gauss' law is circumvented (recall that flux lines decay in a Higgs phase). In the high temperature phase the additional classical quark has finite free energy even in the limit that the classical Higgsing is taken to zero. On the other hand, in the low temperature phase the Polyakov loop vanishes as this Higgsing is taken to zero.

The analysis above shows that $\mathcal{P}^{e f f}=1 / 2$ immediately after the phase transition, and it goes up to $\mathcal{P}^{e f f} \rightarrow 1$ at high temperatures. In fact, $\mathcal{P}^{\text {eff }}$ is exactly the variable $\rho_{1}$ discussed in $\S 5.4$, so the exact behavior of the order parameter is given formally by the first component of $\vec{\rho}$ in (5.32), while the behavior near the transition is given by (5.47), or explicitly to leading orders by $(5.49)$.

\footnotetext{
${ }^{40}$ Of course at any finite $N$ there is some finite $\epsilon_{0}(N)$ that is so small that when $\epsilon \leq \epsilon_{0}(N)$ the path integral receives significant contributions from the entire manifold of 'almost' saddles. However $\epsilon_{0}(N) \rightarrow 0$ as $N \rightarrow \infty$. Consequently, if we take the $N \rightarrow \infty$ limit first, only a single saddle point contributes to the path integral for any $\epsilon$ no matter how small.

${ }^{41}$ Note that this construction is rather similar to the definition of the order parameter of a ferromagnet in 3 dimensions. Naively, the magnetization of the ferromagnet vanishes due to $S O(3)$ symmetry in both the high and low temperature phases. However if we define $|M|_{\epsilon}$ to be the magnetization in the presence of a small magnetic field in the $z$ direction, then $\lim _{\epsilon \rightarrow 0} \lim _{V \rightarrow \infty}|M|_{\epsilon}$ (where $V$ is the volume) is the standard order parameter for the ferromagnet.
} 


\subsection{Results for specific theories}

Before closing this section, we explicitly apply our results to two interesting $3+1$ dimensional theories, pure Yang-Mills theory and the $\mathcal{N}=4 \mathrm{SYM}$ theory. Using the single-particle partition functions given in (3.20), we may write down explicit expressions for the transition temperature using $z\left(x_{H}\right)=$ 1 , for the behavior of the free energy near the transition using (5.51), and for the high temperature behavior using (5.18).

For pure $d=3+1, U(N)$ (or $S U(N)$ ) Yang-Mills theory on an $S^{3}$ of unit radius, the single-particle partition functions are given by

$$
z_{B}(x)=\frac{6 x^{2}-2 x^{3}}{(1-x)^{3}}, \quad z_{F}(x)=0
$$

Solving $z\left(x_{H}\right)=1$, we find that the Hagedorn temperature is given by $x_{H}=2-\sqrt{3}, T_{H}=-1 / \ln (2-\sqrt{3}) \simeq 0.759326$. The free energy below the Hagedorn temperature may be obtained from the partition function $Z$ in (3.8) using (5.57) and recalling that $x=\exp (-1 / T)$. Just above the phase transition, the free energy is given by

$$
\begin{aligned}
\frac{1}{N^{2}} F_{\mathcal{N}=0}\left(T \rightarrow T_{H}^{+}\right)= & -0.9877\left(T-T_{H}\right)-3.004\left(T-T_{H}\right)^{\frac{3}{2}} \\
& -5.980\left(T-T_{H}\right)^{2}+\mathcal{O}\left(\left(T-T_{H}\right)^{\frac{5}{2}}\right)
\end{aligned}
$$

while the high temperature free energy is

$$
F_{\mathcal{N}=0}\left(T \gg T_{H}\right) \rightarrow-\frac{\pi^{2}}{45} N^{2} T^{4} V_{S^{3}},
$$

where $V_{S^{3}}=2 \pi^{2} R^{3}$ is the volume of the sphere (restoring a general value for the $S^{3}$ radius).

For the $d=3+1 \mathcal{N}=4$ SYM theory on an $S^{3}$ of unit radius, the single-particle partition functions are

$$
z_{B}(x)=\frac{6 x+12 x^{2}-2 x^{3}}{(1-x)^{3}}, \quad z_{F}(x)=\frac{16 x^{\frac{3}{2}}}{(1-x)^{3}},
$$

so from $z_{B}\left(x_{H}\right)+z_{F}\left(x_{H}\right)=1$ we find that the Hagedorn temperature is given by ${ }^{42} x_{H}=7-4 \sqrt{3}, T_{H}=-1 / \ln (7-4 \sqrt{3}) \simeq 0.379663$. The free

\footnotetext{
${ }^{42}$ Curiously, $x_{H}$ for the $\mathcal{N}=4$ theory is exactly the square of $x_{H}$ for the pure YangMills theory, and $T_{H}$ in $\mathcal{N}=4 \mathrm{SYM}$ is precisely half of $T_{H}$ in pure Yang-Mills. This arises from the even more peculiar fact that the single-particle partition functions are related by $z_{\mathcal{N}=0}(x)=z_{\mathcal{N}=4}\left(x^{2}\right)$.
} 
energy below the Hagedorn temperature may be obtained from the partition function $Z$ in (3.8) using (5.60), with $x=\exp (-1 / T)$. The free energy immediately above the phase transition temperature is given by

$$
\begin{aligned}
\frac{1}{N^{2}} F_{\mathcal{N}=4}\left(T \rightarrow T_{H}^{+}\right)= & -0.9877\left(T-T_{H}\right)-4.248\left(T-T_{H}\right)^{\frac{3}{2}} \\
& -11.696\left(T-T_{H}\right)^{2}+\mathcal{O}\left(\left(T-T_{H}\right)^{\frac{5}{2}}\right)
\end{aligned}
$$

while the high temperature free energy is

$$
F_{\mathcal{N}=4}\left(T \gg T_{H}\right) \rightarrow-\frac{\pi^{2}}{6} N^{2} T^{4} V_{S^{3}} .
$$

\section{Phase structure at weak coupling}

In this section we turn to an analysis of the phase transition at non-zero 't Hooft coupling ${ }^{43}$. We will see that an arbitrarily small non-zero coupling qualitatively changes the behavior of the transition. In $\S 6.1$ we deduce the most general form of $S_{\text {eff }}(U, \lambda)$ allowed by gauge invariance. In $\S 6.2$ we analyze the structure of $S_{\text {eff }}(U, \lambda)$ in perturbation theory. In $\S 6.3$ we study the structure of the deconfinement transition (discussed in detail for the free theory in the previous section) at weak coupling. In $\S 6.4$ we verify the general analysis of $\S 6.3$ in an exactly solvable toy model. We end in $\S 6.5$ with a description of the implications of our results for the microcanonical ensemble.

\subsection{General properties of the effective action}

For any value of the coupling constant, gauge invariance imposes tight constraints on the form of the effective action $S_{\text {eff }}(\alpha)$ (4.6) for the zero mode of the gauge field; $S_{\text {eff }}(\alpha)$ should be invariant under all space-time gauge transformations that

(1) Are single valued (up to an element of the center of $U(N)$ ) on $\mathcal{M} \times S^{1}$,

(2) Preserve the gauge-fixing conditions (4.1) and (4.2).

\footnotetext{
${ }^{43}$ Some of the key ideas underlying this section and the next one arose in discussions with R. Gopakumar. We thank him in particular for emphasizing the qualitative difference between the two scenarios depicted in figure 4, and for forcing us to understand the order parameter in a clearer fashion.
} 
We will restrict attention to gauge transformations $U(t)$ that are independent of the position on the compact space. Under such a transformation

$$
A_{0} \rightarrow V(t) A_{0} V^{\dagger}(t)-i \partial_{t} V V^{\dagger}
$$

so that

$$
\alpha \rightarrow V(t) \alpha V^{\dagger}(t)-i \partial_{t} V V^{\dagger}
$$

$V(t)$ obeys the condition (2) above (and (6.2) makes sense) only when the right-hand side of (6.2) is independent of time.

Constant (time independent) gauge transformations clearly satisfy the requirements (1) and (2) above. Consequently, $S_{\text {eff }}(\alpha)$ is invariant under $\alpha \rightarrow V \alpha V^{\dagger}$. We may use this invariance to diagonalize $\alpha$. Once this has been done, we consider the further gauge transformations

$$
V(t)=e^{i t D}
$$

where $D$ is a diagonal matrix, whose eigenvalues are all integral multiples of $2 \pi / \beta . \alpha$ transforms under the gauge transformation generated by (6.3) as $\alpha \rightarrow \alpha+D$. This implies that $S_{\text {eff }}(\alpha)$ is invariant under separate shifts of any of the eigenvalues of $\alpha$ by multiples of $2 \pi / \beta$. It follows from these invariances that $S_{\text {eff }}$ is really a function only of $\operatorname{tr}\left(\mathrm{U}^{\mathrm{n}}\right)$ (for all $n$ ) where $U=e^{i \beta \alpha}$ (see (4.12)) is the zero mode holonomy around the time circle.

Finally, consider $V(t)=e^{\frac{2 \pi i k t}{\beta N}}$ where $k$ is an integer if the gauge group is $S U(N)$, and arbitrary for $U(N)$. $V(t)$ obeys the single-valuedness con-

dition (1), as $e^{\frac{2 \pi i k}{N}}$ belongs to the center of the gauge group. Under the gauge transformation generated by $V(t), \alpha \rightarrow \alpha+\frac{2 \pi k}{N \beta}$. Consequently, $S_{\text {eff }}$ should be invariant under $U \rightarrow e^{\frac{2 \pi i k}{N}} U$, for all integers $k$ if the gauge group is $S U(N)$, and for any $k$ for $U(N)$. In the limit $N \rightarrow \infty$ the two cases coincide, and $S_{\text {eff }}(U)$ must be invariant under $U \rightarrow e^{i \theta} U$ for arbitrary $\theta$. Putting everything together, we conclude that $S_{\text {eff }}$ may depend on $U$ only in combinations of the form

$$
\operatorname{tr}\left(\mathrm{U}^{\mathrm{n}_{1}}\right) \cdots \operatorname{tr}\left(\mathrm{U}^{\mathrm{n}_{\mathrm{k}}}\right) \operatorname{tr}\left(\mathrm{U}^{-\mathrm{n}_{1}-\cdots-\mathrm{n}_{\mathrm{k}}}\right) .
$$

\subsection{The general form of the effective action in perturbation theory}

In large $N$ perturbation theory, $S_{\text {eff }}$ is generated by planar vacuum diagrams obtained by integrating out the massive modes. In this calculation, $\alpha$ is a background field, which appears diagrammatically via external line 
insertions on the index loops of diagrams. Planar diagrams at order $\lambda^{k-1}$ include $(k+1)$ index loops, each of which leads to a trace in the final result (which may contain arbitrarily many factors of $\alpha$ ). From the discussion of the previous subsection, the only terms with $(k+1)$ traces allowed by gauge invariance take the form (6.4). It follows that the most general form of the planar contribution to $S_{\text {eff }}$ in perturbation theory is

$$
\begin{aligned}
S_{e f f}(U)= & N^{2}\left[\sum_{n} m_{n}^{2}(x)\left|u_{n}\right|^{2}+\lambda \sum_{m, n} F_{m, n}(x)\left(u_{m} u_{n} u_{-n-m}+c . c .\right)\right. \\
& \left.+\lambda^{2} \sum_{m, n, p} F_{m, n, p}(x)\left(u_{m} u_{n} u_{p} u_{-m-n-p}+c . c .\right)+\cdots\right]
\end{aligned}
$$

where we have defined $u_{n} \equiv \operatorname{tr}\left(\mathrm{U}^{\mathrm{n}}\right) / \mathrm{N}$ (note that $u_{n}^{*}=u_{-n}$ and $u_{0}=1$ ). This agrees, of course, with the quadratic form of the one-loop effective action that we found in sections 3 and 4 . Also, the $u_{n}$ coincide with the variables $\rho_{n}$ used in $\S 5$ for eigenvalue distributions which are symmetric about $\theta=0$, so the values of $m_{n}^{2}$ in the free field theory may be read off from equation (5.7).

\subsection{Possible phase structures at weak coupling}

Recall from $\S 5$ that, at $\lambda=0, u_{1}$ is massless at the phase transition temperature $T_{H}$, while the other $u_{n}$ 's are all massive. Consequently, $u_{1}$ will continue to be the lightest mode in the vicinity of the phase transition also at weak coupling. Thus, for analyzing the phase transition at weak coupling, it is useful to obtain an effective action for $u_{1}$ by integrating out the $u_{n}$ 's with $n>1^{44}$. It follows from (6.5) that the leading large $N$ terms in the resulting effective action are of the form

$$
S_{e f f}\left(u_{1}\right)=N^{2}\left(m_{1}^{2}(x, \lambda)\left|u_{1}\right|^{2}+\sum_{n=2}^{\infty} \lambda^{2 n-2} B_{n}(x, \lambda)\left|u_{1}\right|^{2 n}\right),
$$

where $m_{1}^{2}$ and the $B_{n}$ 's are functions of the temperature and power series in $\lambda$ starting (generically) from a $\lambda^{0}$ term.

In particular, to $\mathcal{O}\left(\lambda^{2}\right)$ we have

$$
S_{e f f}\left(u_{1}\right)=N^{2}\left(m_{1}^{2}\left|u_{1}\right|^{2}+b\left|u_{1}\right|^{4}\right),
$$

\footnotetext{
${ }^{44}$ As we are interested in the large $N$ limit, it is sufficient to integrate out these modes classically.
} 
where $b(x)=B_{2}(x, 0) \lambda^{2}$ is a function of temperature which is generically non-zero. It is not difficult to compute $B_{2}(0)$ starting from (6.5). The only terms in (6.5) that contribute are

$$
S_{e f f}^{\prime}(U)=N^{2}\left(m_{1}^{2}\left|u_{1}\right|^{2}+m_{2}^{2}\left|u_{2}\right|^{2}+\lambda I\left(u_{-2} u_{1}^{2}+u_{2} u_{-1}^{2}\right)+\lambda^{2} A\left|u_{1}\right|^{4}\right) .
$$

At this order, $u_{2}$ may be integrated out from (6.8) by setting it to be equal to its classical value $u_{2}=-I \lambda u_{1}^{2} / m_{2}^{2}{ }^{45}$, yielding (6.7) with $b=\left(A-I^{2} / m_{2}^{2}\right) \lambda^{2}$. Note that because the eigenvalue density (6.9) has to be non-negative everywhere, our expressions for the effective action (6.6) and (6.7) are valid only for $u_{1}$ such that $\left|u_{1}\right| \leq \frac{1}{2}+\mathcal{O}(\lambda)$.

As in $\S 5$, the one-loop contribution to the free energy coming from integrating over $u_{1}$ diverges at the temperature $T_{H}(\lambda)$ at which $m_{1}^{2}(x, \lambda)$ goes to zero and $u_{1}$ becomes massless. For any value of the coupling, at leading order in the distance from this temperature, we have $m_{1}^{2} \simeq K\left(T_{H}(\lambda)-T\right)$ for some positive constant $K$. This divergence signals a Hagedorn behavior of the single-particle spectrum of the theory with Hagedorn temperature $T_{H}$ (computable in perturbation theory), so this behavior persists (at least in the microcanonical ensemble) even at non-zero 't Hooft coupling.

As the saddle point at $u_{1}=0$ is unstable for $T>T_{H}$, the theory described by (6.7) clearly undergoes a phase transition (to another saddle point) at some $T \leq T_{H}$. Whether this phase transition occurs at $T<T_{H}$ or $T=$ $T_{H}$ depends on whether the value of $b$ (defined in (6.7)) at the Hagedorn temperature $T_{H}$ is positive or negative, as we now argue in detail ${ }^{46}$. The formulas in the remainder of this section are all correct only to leading order in $b$ (or in $\lambda$ ).

First, consider the case $b>0$. For $T<T_{H}, u_{1}=0$, corresponding to the uniform eigenvalue distribution, is clearly a global minimum of the effective action (6.7). For $T>T_{H}$, however, $u_{1}=0$ is unstable and $S_{\text {eff }}$ is minimized at $\left|u_{1}\right|^{2}=\left|m_{1}\right|^{2} / 2 b$. The value of the effective action at this minimum is $S_{e f f}=-N^{2}\left|m_{1}\right|^{4} / 4 b$, which is of order $\left(T-T_{H}\right)^{2}$, and so the phase transition at $T=T_{H}$ is of second order. As the temperature is raised above $T_{H}$, the eigenvalue distribution smoothly becomes non-uniform until we reach $\left|u_{1}\right|^{2}=1 / 4$; this occurs at $m_{1}^{2}=-b / 2$. At this point, $\rho(\theta)$ vanishes at some $\theta$ and we have reached the boundary of the space of eigenvalue distributions

${ }^{45}$ Thus setting the eigenvalue distribution to

$$
\rho(\theta)=\frac{1}{2 \pi}\left(1+2\left|u_{1}\right| \cos (\theta+\alpha)-\frac{2 \lambda I}{m_{2}^{2}}\left|u_{1}\right|^{2} \cos (2 \theta+2 \alpha)\right)
$$

for some $\alpha$.

${ }^{46}$ See [68] for a similar discussion in a related context. 


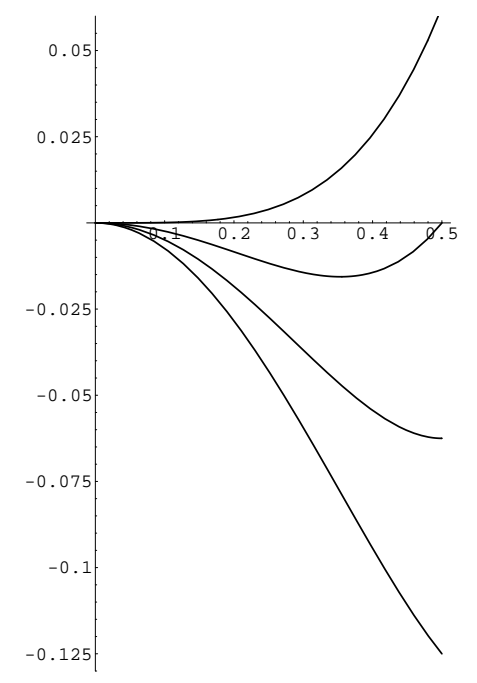

Figure 2: Plots of $S_{\text {eff }}\left(u_{1}\right)$ as a function of $u_{1}$ for small positive $b$, in units of $N^{2} b$, at several values of $m_{1}^{2}$ (from top to bottom): $m_{1}^{2}=0$ (Hagedorn temperature and first phase transition temperature), $m_{1}^{2}=-\frac{b}{4}, m_{1}^{2}=-\frac{b}{2}$ (second phase transition temperature), and $m_{1}^{2}=-\frac{3 b}{4}$.

and the edge of the validity of (6.7). As the temperature is further raised the eigenvalue distribution develops a gap on the circle, and the theory undergoes a further phase transition similar to the Gross-Witten transition [41]. This second phase transition (at $T=T_{H}+\frac{b}{2 K}$ ) is of third order. The behavior above this second transition temperature is no longer captured by the effective action (6.6), and the full action is required to analyze it.

In the case where $b$ is negative, $S_{\text {eff }}$ develops a new local minimum at $\left|u_{1}\right|=\frac{1}{2}$ (the boundary of our order parameter space) when $m_{1}^{2}<|b| / 2$; note this happens below the Hagedorn temperature. When $m_{1}^{2}>|b| / 4$, the free energy at this new local minimum is positive, and the saddle point $\left|u_{1}\right|=\frac{1}{2}$ is disfavored compared to $u_{1}=0$. However, when we raise the temperature to $m_{1}^{2}<|b| / 4$, the free energy at $\left|u_{1}\right|=\frac{1}{2}$ becomes negative, and so this saddle dominates over the $u_{1}=0$ saddle point. Consequently, at $T=T_{H}-\frac{|b|}{2 K},\left|u_{1}\right|$ jumps discontinuously from zero to $\frac{1}{2}$ and the theory undergoes a first order phase transition. When the temperature is further raised, the eigenvalue distribution develops a break on the circle and the theory is no longer described by (6.6). This behavior is qualitatively similar 


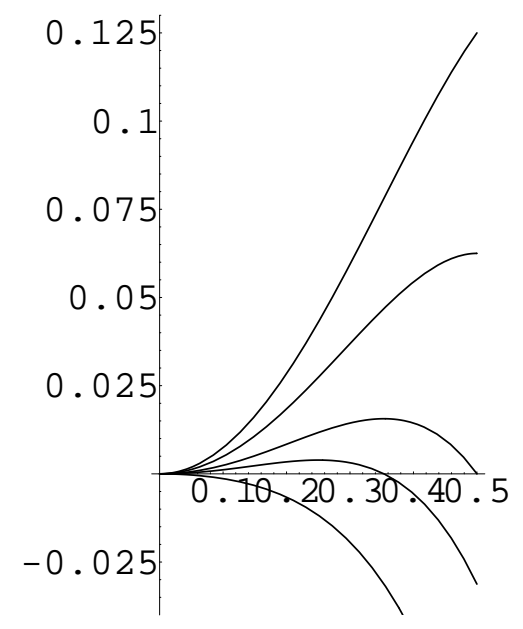

Figure 3: Plots of $S_{\text {eff }}\left(u_{1}\right)$ as a function of $u_{1}$ for small negative $b$, in units of $N^{2}|b|$, at several values of $m_{1}^{2}$ (from top to bottom) : $m_{1}^{2}=\frac{3|b|}{4}, m_{1}^{2}=\frac{|b|}{2}$ (new phase nucleated), $m_{1}^{2}=\frac{|b|}{4}$ (first order phase transition temperature), $m_{1}^{2}=\frac{|b|}{8}$, and $m_{1}^{2}=-\frac{|b|}{8}$.

to that of the free theory which we analyzed in $\S 5$, except that the phase transition now happens below the Hagedorn temperature $T_{H}$.

If $b$ vanishes exactly then the higher order terms in (6.6) are required for analyzing the phase transition, but generically $b$ will not vanish at the Hagedorn temperature. In our discussions above we assumed that $b$ was constant in a range of temperatures near the Hagedorn temperature; this is consistent at weak coupling since the range of temperatures which is relevant for the discussions above is of order $\lambda^{2}$, so the changes in $b$ within this range are of higher order in $\lambda$. 


\subsection{Toy model}

The varieties of behavior described in the previous subsection may be illustrated using a simple toy example, given by the matrix model

$$
Z(\beta)=\int[d U] \exp \left[-\left(|\operatorname{tr}(\mathrm{U})|^{2}\left(\mathrm{~m}_{1}^{2}-1\right)+\mathrm{b}|\operatorname{tr}(\mathrm{U})|^{4} / \mathrm{N}^{2}\right)\right] .
$$

Based on the previous discussion, we expect this toy model to give a good picture of the behavior of the full theory for small $\lambda$ near the phase transition, since the $u_{n}$ 's for $|n|>1$ are all small there. Readers who believe our general discussion above may skip directly to the next subsection.

Our toy model may be solved exactly using the methods of $\S 5.4$. Since the action in (6.10) depends only on $\operatorname{tr}(\mathrm{U})$ and not on traces of higher powers of $U$, the possible forms of the saddle point eigenvalue distribution for the integral (6.10) (up to an overall shift in $\theta$ ) are the same as those in [41] (and in our truncated model in $\S 5$ ). The possible distributions may be parametrized by a positive number $g$, and they are given by one of the two expressions

$$
\begin{aligned}
& \rho(\theta)=\frac{1}{2 \pi}\left(1+\frac{2}{g} \cos (\theta)\right) \quad g \geq 2, \\
& \rho(\theta)=\frac{2}{\pi g} \cos \left(\frac{\theta}{2}\right) \sqrt{\frac{g}{2}-\sin ^{2} \frac{\theta}{2}} \quad g \leq 2,
\end{aligned}
$$

where for the matrix model (6.10) we have

$$
g^{-1}=u_{1}\left(1-m_{1}^{2}-2 b u_{1}^{2}\right)
$$

with

$$
u_{1}=\frac{\operatorname{tr}(\mathrm{U})}{\mathrm{N}}=\int d \theta \rho(\theta) \cos (\theta) .
$$

Note that $u_{1}$ is real since we assumed in (6.11) that the eigenvalue distribution is symmetric about $\theta=0$.

We search for simultaneous solutions to (6.11), (6.12) and (6.13). It is easy to check that the constant distribution $u_{1}=0(g=\infty, \rho(\theta)=1 / 2 \pi)$ is always a solution; we call this solution Phase I. The free energy in Phase $\mathrm{I}$ is zero. From the discussion of $\S 5$ we know that this solution is a local minimum when $m_{1}^{2}>0$.

An additional solution (Phase II) is obtained by using the first distribution in (6.11) (with $g \geq 2$ ), upon solving (6.12) and (6.13) for this case. This gives

$$
u_{1}=\left(1-m_{1}^{2}\right) u_{1}-2 b u_{1}^{3},
$$


with the solution

$$
u_{1}^{2}=-\frac{m_{1}^{2}}{2 b} .
$$

Obviously, this only makes sense if $m_{1}^{2} / b \leq 0$; consistency of the solution also requires

$$
g^{-2}=\left|\frac{m_{1}^{2}}{2 b}\right| \leq \frac{1}{4}
$$

so this phase exists and is a local minimum for $b>0$ and $-b / 2 \leq m_{1}^{2} \leq 0$ (such a phase exists also for $m_{1}^{2}>0$ and $b<0$, but in that case its free energy is positive and so this phase is sub-dominant compared to Phase I). The free energy $F=-T \ln (Z)$ evaluated on this saddle point is

$$
F(T)=-\frac{N^{2} T m_{1}^{4}}{4 b},
$$

which is quadratic in $\left(T-T_{H}\right)$ near the Hagedorn temperature (where $m_{1}^{2} \propto$ $\left.T_{H}-T\right)$.

To find solutions using the second distribution in (6.11) (with $g \leq 2$ ), we must once again solve (6.12) and (6.13) with the appropriate distribution, and we find

$$
u_{1}=1-\frac{g}{4}=1-\frac{1}{4\left(u_{1}\left(1-m_{1}^{2}\right)-2 u_{1}^{3} b\right)} .
$$

Given the solution for $u_{1}$, the free energy evaluated on this saddle point is

$$
F(T)=N^{2} T\left[\left(m_{1}^{2}-1\right) u_{1}^{2}+b u_{1}^{4}+\frac{1}{4}-\frac{1}{2} \ln \left(2\left(1-u_{1}\right)\right)\right]
$$

(the first two terms come from the potential in (6.10) evaluated on the saddle, while the remaining two terms come from the measure).

The exact solution to the quartic equation (6.18) is easily obtained, though it is not particularly illuminating. It may be checked that there are no physical solutions for $m_{1}^{2}>-\frac{b}{2}$, while for $m_{1}^{2}=-\frac{b}{2}$ we find $u_{1}=\frac{1}{2}$. The discussion of the previous subsection suggests (and we will demonstrate this below) that this solution represents the phase transition point from Phase II to Phase III when $b>0$, and a phase nucleation point (the creation of a new phase at the boundary) when $b<0$.

To understand the behavior near this value of $m_{1}^{2}$, we analyze the solutions to (6.18) in the neighborhood of this special point. For this purpose we introduce a new variable $y \equiv-m_{1}^{2}-\frac{b}{2}$ which is linear in $\delta T$, the increment over the phase transition (or nucleation) temperature. We also introduce a variable $\delta \equiv \frac{b}{2}\left(4 u_{1}^{2}-1\right)$ that measures the deviation of $u_{1}$ from its value at 
the phase transition (or nucleation) point. In terms of these variables, (6.18) may be rewritten as

$$
2 \sqrt{1+\frac{2}{b} \delta}\left(1-\frac{1}{2} \sqrt{1+\frac{2}{b}} \delta\right)(1+y-\delta)=1 .
$$

The explicit solution for $y$ in terms of $\delta$ follows immediately from this; this solution may be expanded in a power series in $\delta$ and inverted to obtain ${ }^{47}$

$$
\delta=y-\frac{y^{2}}{b^{2}}+\frac{2+b}{b^{4}} y^{3}-\left(\frac{5}{b^{6}}+\frac{5}{b^{5}}+\frac{9}{4 b^{4}}\right) y^{4}+\mathcal{O}\left(y^{5}\right) .
$$

Using our definition $\delta \equiv \frac{b}{2}\left(4 u_{1}^{2}-1\right)$ we now find

$$
u_{1}=\frac{1}{2}\left(1+\frac{1}{b} y-\frac{2+b}{2 b^{3}} y^{2}+\frac{4+4 b+b^{2}}{2 b^{5}} y^{3}+\mathcal{O}\left(y^{4}\right)\right)
$$

and inserting this into (6.19) we obtain

$$
\begin{aligned}
F(T) & =N^{2} T\left[-\frac{b}{16}-\frac{y}{4}-\frac{y^{2}}{4 b}+\frac{1}{6 b^{3}} y^{3}+\mathcal{O}\left(y^{4}\right)\right] \\
& =N^{2} T\left[-\frac{1}{4 b}\left(y+\frac{b}{2}\right)^{2}+\frac{1}{6 b^{3}} y^{3}+\mathcal{O}\left(y^{4}\right)\right] \\
& =N^{2} T\left[-\frac{m_{1}^{4}}{4 b}+\frac{1}{6 b^{3}} y^{3}+\mathcal{O}\left(y^{4}\right)\right] .
\end{aligned}
$$

In summary, Phase I exists for all values of $m_{1}$ and $b$. Its free energy is zero. Phase II exists when $0<-m_{1}^{2} / b \leq 1 / 2$. Its free energy is $F=$ $-N^{2} T m_{1}^{4} / 4 b$. Finally, Phase III exists whenever there is a solution with $u_{1} \geq 1 / 2$ to (6.18) and has a free energy given by (6.19) (for $b>0$ this phase exists when $-m_{1}^{2} / b \geq 1 / 2$, and for $m_{1}^{2}$ close to $-b / 2$ it has a free energy given by (6.23)). Recall that $m_{1}^{2}=K\left(T_{H}-T\right)$ where $K$ is positive.

It is now clear that the dynamics of our toy model near the Hagedorn temperature $m_{1}^{2}\left(T_{H}\right)=0$ depends sensitively on the sign of $b$ at this temperature. First consider $b>0$. For $T<T_{H}\left(m_{1}^{2}>0\right)$, Phase I with $u_{1}=0$ is the only saddle point, and it has vanishing free energy. As $T$ rises above $T_{H}$ (for small negative $m_{1}^{2}$ ) a new phase (Phase II) comes into existence. Simultaneously Phase I becomes unstable, and the theory executes a second order phase transition into Phase II at $T=T_{H}$. Finally, at a still higher

\footnotetext{
${ }^{47}$ The following three formulas are only correct for $b>0$; for $b<0$ there is another solution to (6.20) which gives the leading contribution [5]. We thank L. Alvarez-Gaume, C. Gomez, H. Liu and S. Wadia for pointing this out to us.
} 
temperature $\left(m_{1}^{2}=-b / 2\right)$ Phase II evolves continuously into Phase III; this transition is of third order, due to (6.17), (6.23), and the fact that $y$ in (6.23) is linear in $\delta T$. As the temperature is raised further, the theory remains in Phase III.

On the other hand, if $b<0$, Phase I is the only saddle point for $m_{1}^{2} \geq$ $-b / 2$. At this temperature (below the Hagedorn temperature) a new phase comes into existence. As the temperature is further raised, this new phase splits into two different saddle points (Phase II and Phase III). Phase II has positive free energy whenever it exists, and is always unstable. Phase III is locally stable; however its free energy is positive in the neighborhood of $m_{1}^{2}=-b / 2$, and so (at this point) this saddle is sub-dominant compared to Phase I. Upon further raising the temperature, $F(T)$ of Phase III becomes negative at some positive value of $m_{1}^{2}$, which may be obtained by solving (6.18) and (6.19) simultaneously for $u_{1}$ and $m_{1}^{2}$; note that this happens below the Hagedorn temperature. Finally, as the temperature is raised above the Hagedorn temperature, Phase II disappears and Phase I becomes unstable, so the theory remains in Phase III at all higher temperatures.

In conclusion, the exact solution of the toy model (6.10) verifies, in complete detail, the general results of the previous subsection.

\subsection{Density of states as a function of energy}

In the previous subsections we discussed the canonical partition function as a function of temperature for Yang-Mills theories at weak coupling. We found three qualitatively different classes of $Z(\beta)$ depending on whether $b$ (see (6.7)) is negative, zero or positive. In this subsection we will qualitatively describe the corresponding Yang-Mills theories in the microcanonical ensemble, seeing how the density of states depends on the energy in each of these three different classes of models.

In order to make contact with the canonical ensemble discussed in previous subsections, we will find it convenient to characterize the microcanonical density of states $S(E)=\ln (\rho(E))$ in a rather peculiar way. We will find it convenient to plot the logarithm of the effective temperature $\ln (T(E))=$ $-\ln (\partial S(E) / \partial E)$ as a function of $\ln (E)^{48}$; such plots were used for very similar purposes in [9].

The behavior of the density of states (or $\frac{\partial S}{\partial E}$ ) as a function of temperature may be deduced directly from the behavior of $F(T)$ using the relation

\footnotetext{
${ }^{48}$ We thank R. Gopakumar for suggesting this to us.
} 


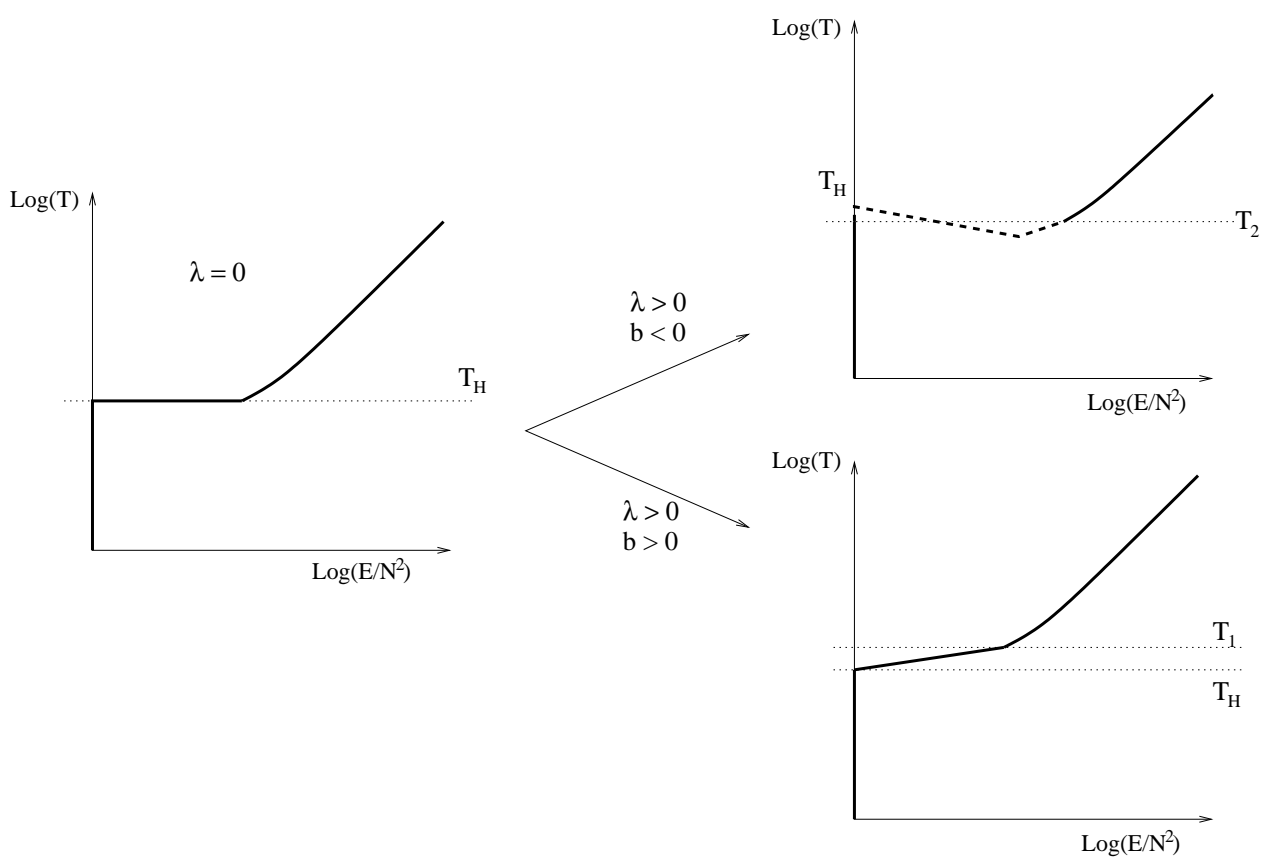

Figure 4: Plot of $\log (T)$ as a function of $\log (E)$ (for energies of order $N^{2}$, in the microcanonical ensemble), for $\lambda=0$ and for $\lambda>0$ with $b>0$ and $b<0$.

$E=\partial_{\beta}(\beta F)$. However, this information is incomplete where there are first order phase transitions, since these correspond to temperatures at which the energy jumps discontinuously in going from one phase to another (non-zero latent heat). For the case $\lambda=0$, we have seen that the first order transition is a Hagedorn transition, so we may conclude that for microcanonical energies intermediate between the two phases, the density of states is Hagedorn, corresponding to a horizontal line in the graph of $\ln (T)$ as a function of $\ln (E)$. Thus, the $F(T)$ behavior depicted in figure 1 leads to the microcanonical behavior shown on the left in figure 4 . For small $\lambda>0$, the density of states should be a small perturbation of this behavior, and we may deduce from the results of $\S 6.3$ that the qualitatively different behavior obtained for $b>0$ and $b<0$ corresponds to whether the flat Hagedorn region in the $\lambda=0$ plot tilts upwards $(b>0)$, resulting in two continuous phase transitions at $T_{H}$ and some higher temperature $T_{1}$, or downwards $(b<0)$, preserving the first order transition (dashed lines in figure 4 indicate points not accessible in the canonical ensemble).

The plots in figure 4 correspond to energies of order $N^{2}$, and they are drawn in the large $N$ limit where the canonical ensemble exhibits sharp tran- 
sitions and the boundaries between phases in the microcanonical ensemble are distinct. To understand in more detail the phases available in the microcanonical ensemble at general energies (including energies of order one), it is convenient to stretch out the low energy regions of the three diagrams for large but finite $\mathrm{N}$, as we do in figures 5,6 , and 7 below. ${ }^{49}$

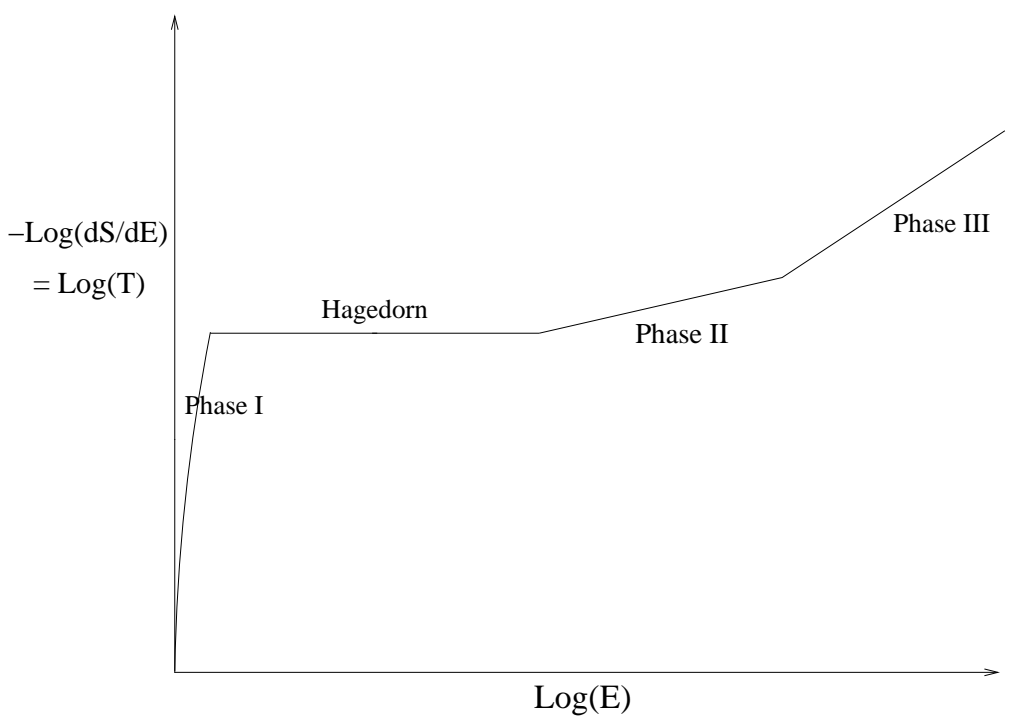

Figure 5: Plot of $\log (T)$ as a function of $\log (E)$ (in the microcanonical ensemble) when $b>0$.

In the case $b>0$ this plot is displayed in figure 5 . As is apparent from the figure, at any given temperature the theory has exactly one phase available to it; this is consistent with the behavior described by the series of LandauGinzburg diagrams in figure 2 and with the analysis of the toy model of the previous section. For $T<T_{H}$ the theory is in Phase I. At $T_{H}$, the theory undergoes a second order Hagedorn phase transition, emerging into Phase II (the energy after the transition is of order $N^{2}$ ). At a still higher temperature, the theory undergoes yet another continuous phase transition into Phase III.

Note that Phase I corresponds to $u_{1}=0$, Phase II corresponds to the minimum of (for instance) the second plot in figure 2, and Phase III is at the boundary of the configuration space of eigenvalue distributions.

The density of states of the free theory may be obtained from the limit

\footnotetext{
${ }^{49}$ Strictly speaking, the boundaries between phases are smoothed out when $N$ is finite, but for large enough $N$ these should look sharp as shown.
} 


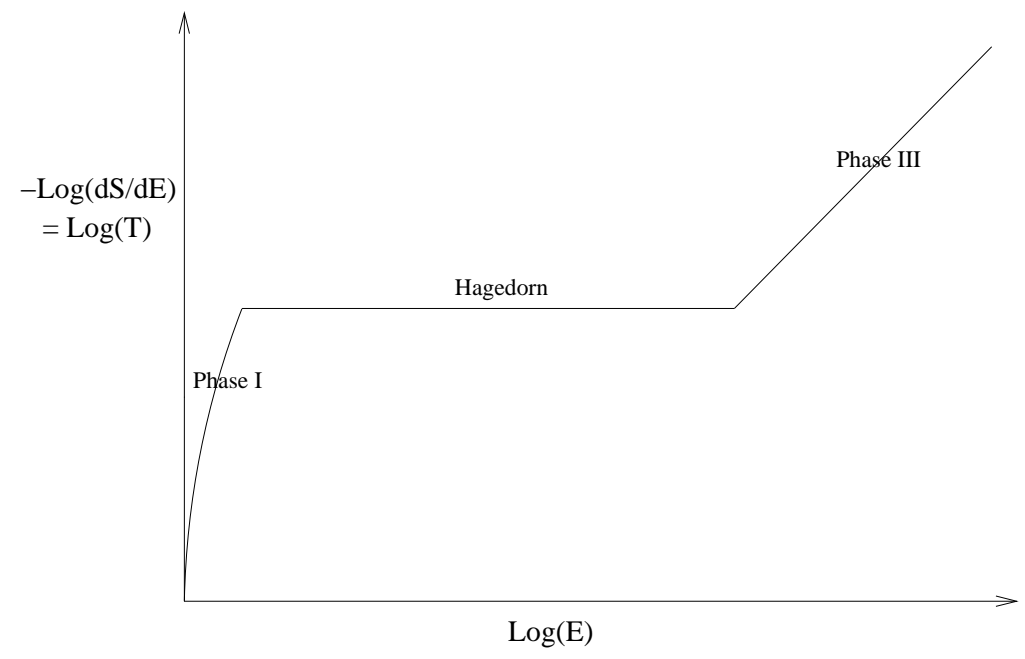

Figure 6: Plot of $\log (T)$ as a function of $\log (E)$ (in the microcanonical ensemble) in the free Yang-Mills theory.

$b \rightarrow 0$ of the previous discussion; the relevant plot takes the form shown in figure 6. As is apparent from this figure, the free theory makes a phase transition (of first order) directly from Phase I to Phase III at the Hagedorn temperature, in agreement with the analysis of $\S 5$.

When $b<0$, we believe ${ }^{50}$ that the density of states is characterized by the plot displayed in figure 7 . The thermodynamics induced by the density of states plotted in figure 7 is consistent with the behavior described by the Landau Ginzburg plots in figure 3. In particular, the five graphs shown in figure 3 (from top to bottom) should be taken to represent $S_{\text {eff }}\left(u_{1}\right)$ for $T<T_{1}, T=T_{1}, T=T_{2}, T_{2}<T<T_{H}$ and $T>T_{H}$, respectively. Phase I lies at $u_{1}=0$ in figure 3 . Phase II is the unstable maximum in the $3 \mathrm{rd}$ and 4th graphs in figure 3, and Phase III is the saddle point at the boundary $u_{1}=\frac{1}{2}$. Note that, according to both figures 7 and 3 , Phase III exists only for $T>T_{1}$, and Phase II exists only for $T_{1}<T<T_{H}$.

In ending this section we would like to emphasize that adding interactions has two qualitatively different effects on the spectrum of weakly coupled

\footnotetext{
${ }^{50}$ As noted above, when a theory undergoes a first order transition, $Z(\beta)$ is insufficient to reproduce the full density of states of the theory. In order to obtain figure 7 we use one additional assumption, that the unstable saddle points appearing in the Landau Ginzburg diagram of figure 3 (see also the toy model of the previous subsection) may be interpreted as an unstable phase (of negative specific heat) at the corresponding temperature, in the microcanonical ensemble.
} 


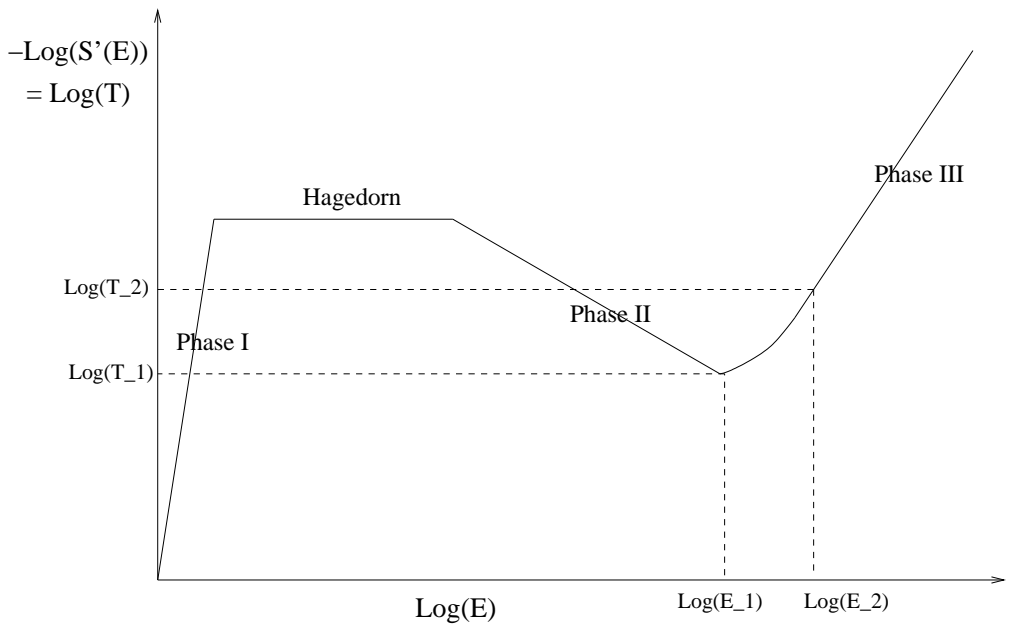

Figure 7: Plot of $\log (T)$ as a function of $\log (E)$ (in the microcanonical ensemble) when $b<0$.

Yang-Mills theories. First, single-trace states involving a number of fields which is much smaller than $N$ pick up corrections to their energy, starting at first order in $\lambda$ (for conformal field theories on $S^{d-1}$ these corrections are equivalent to the anomalous dimensions of the single-trace operators). These corrections result in a renormalization of $m_{1}^{2}(T)$, and hence of the Hagedorn temperature. However, the physics of the phase transition (and, in particular, the crucial sign of the coefficient $b$ ) is governed by a different effect, which is the deviation from Hagedorn-type behavior in states which involve a number of fields of order $N^{2} .{ }^{51}$ This effect appears even in the free Yang-Mills theory, and it appears to be modified in a qualitatively important fashion by coupling constant effects at $\mathcal{O}\left(\lambda^{2}\right)$, as shown in figure 4 . These modifications determine the sign of $b$ and the nature of the phase transition at finite coupling.

\section{Extrapolation to strong coupling and the dual description}

In this section we will present possible extrapolations of the results of section 6 to strong coupling. We will also discuss the possible interpretation of

\footnotetext{
${ }^{51}$ When the number of fields is of order $\sqrt{N}$ or larger, single-trace and multi-trace states mix, and one cannot distinguish between them. This was highlighted recently in the study of the effects of non-planar graphs in the Yang-Mills/plane wave duality [25].
} 
these results in a stringy dual description. Much of this section is rather speculative; we will present some questions and outline possible answers.

\subsection{Possible phase diagrams for large $N$ Yang-Mills theories}

Consider the deconfining transition of a $d$-dimensional confining large $N$ Yang-Mills theory on a compact space of size $R$, at large values of $R \Lambda_{Q C D} \rightarrow$ $\infty$. As we reviewed in $\S 2.2$, this transition may be either of first order (as seems to be the case for pure Yang-Mills theory) or of second order (in which case, for $d=4$ it must be in the universality class of the $d=3 \mathrm{XY}$ model).

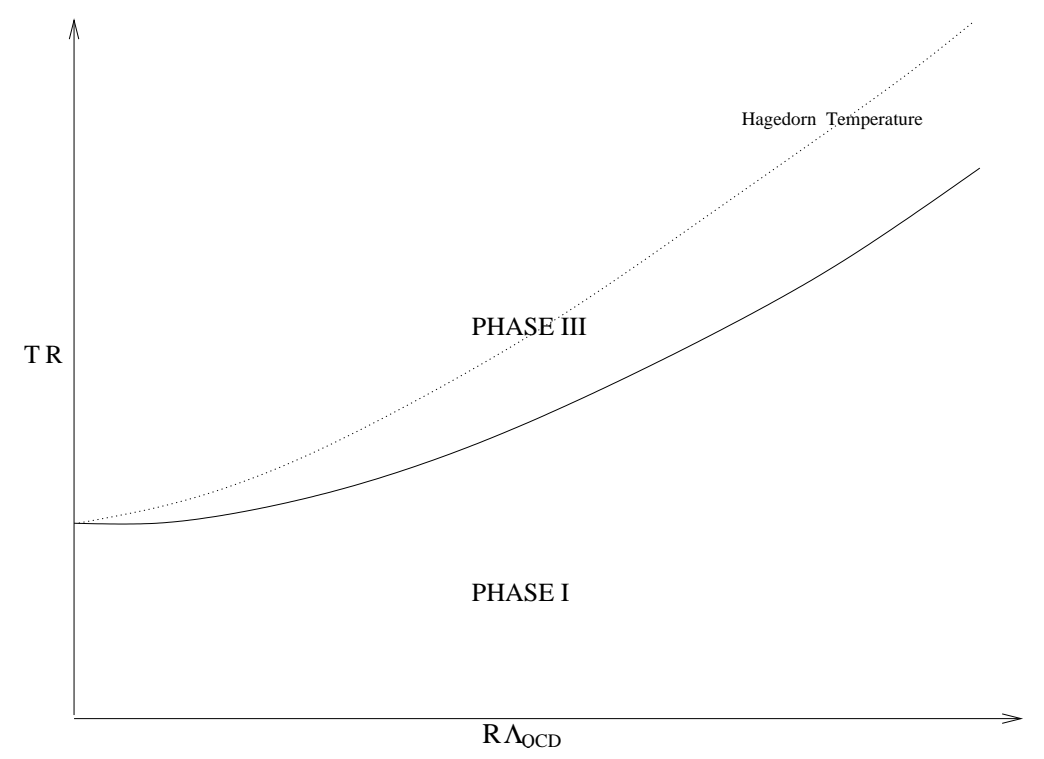

Figure 8: The simplest phase diagram for a compactified confining theory with negative $b$ and a first order phase transition at $R=\infty$. Only solid lines represent phase transitions.

On the other hand, as we saw in the previous section, at weak coupling (small $R \Lambda_{Q C D}$ ) the transition may be of either first or second order, depending on the (perturbatively computable) sign of $b$ for the theory in question. For each of the four possibilities for the order of the phase transitions at large and small $R \Lambda_{Q C D}$ we have sketched the simplest possible interpolation between the two limits, and the corresponding phase diagrams are displayed in figures $8-11$. Recall that Phase I is a confined phase, characterized by a uniform distribution of eigenvalues for the holonomy matrix $U{ }^{52}$ On the other

\footnotetext{
${ }^{52}$ In confining theories away from weak coupling, when the size of the compact space
} 


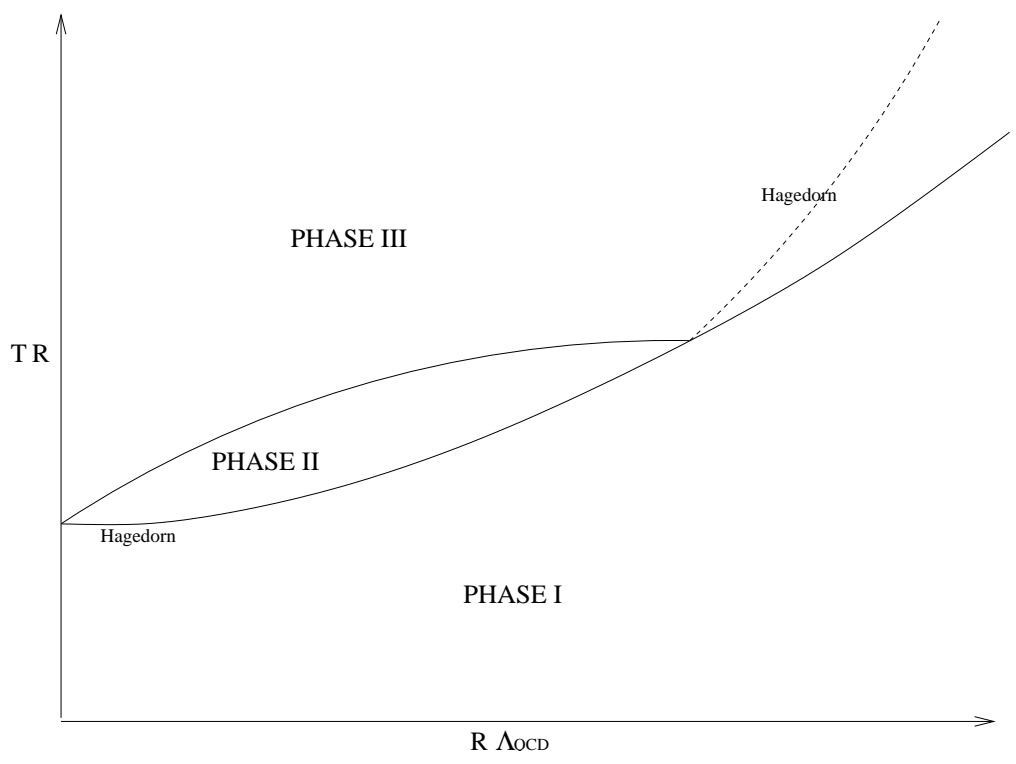

Figure 9: The simplest phase diagram for a compactified confining theory with positive $b$ and a first order phase transition at $R=\infty$. Only solid lines represent phase transitions.

hand Phases II and III are both deconfined phases, which are distinguished by the fact that in Phase II the eigenvalue distribution is non-vanishing on the whole unit circle, while it vanishes outside an interval in Phase III. ${ }^{53}$

Note that in each of the figures 8-11 all phase transition temperatures at strong coupling are of $\mathcal{O}\left(\Lambda_{Q C D}\right)$; consequently, in this regime the dimensionless phase transition temperature $T R$ increases linearly with the coupling parameter $R \Lambda_{Q C D}$.

Since lattice data indicates that 3+1-dimensional pure Yang-Mills theory undergoes a single first order deconfining phase transition at large $N$, the

becomes large compared to the scale set by $\Lambda_{Q C D}$, it is not as useful to focus on the zero mode of the gauge field, since other modes become light compared to $\Lambda_{Q C D}$. However, while $S_{\text {eff }}(U)$ may be less relevant in these more general cases, we can consider instead the effective action $S_{\text {eff }}(U(y)$ ), where $U(y)$ is a spatially-dependent unitary matrix given by the Wilson loop around the thermal circle at a point $y$ (see [34] for a related discussion). For large $N$ theories, we expect that the saddle point configurations for every temperature (in all phases) should be spatially homogeneous, so that the corresponding $U(y)$ is constant. Thus, we may still characterize the various phases by a single eigenvalue distribution corresponding to this constant $U$.

${ }^{53}$ Alternatively, as mentioned for instance in [33], they can be distinguished by the behavior of the Polyakov loops for higher representations of the gauge group, such as the $k$ 'th product of fundamental representations for large $k$. 


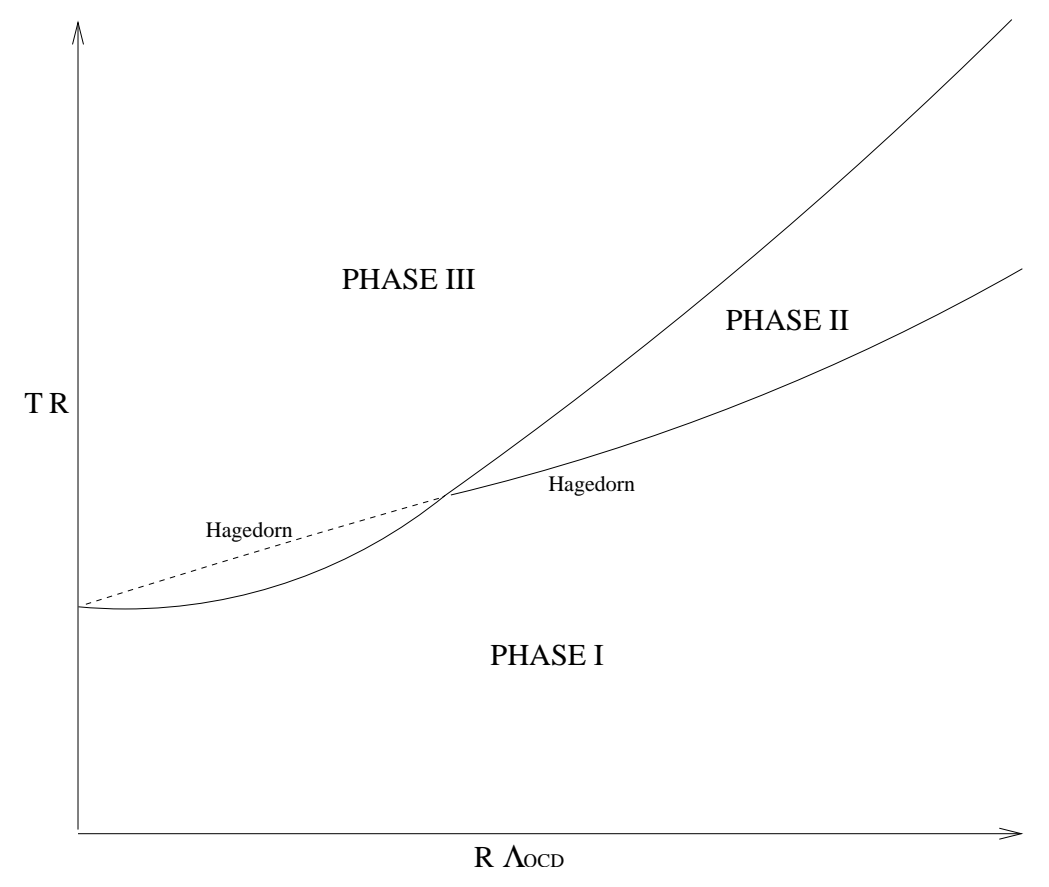

Figure 10: The simplest phase diagram for a compactified confining theory with negative $b$ and a second order phase transition at $R=\infty$. Only solid lines represent phase transitions.

simplest possibilities for the phase diagram of the $3+1$-dimensional large $N$ pure Yang-Mills theory on a compact space such as $S^{3}$ are depicted in either figure 8 or figure 9 ; as above, the value of $b$ for this theory (which we will report on in [3]) will distinguish between these two options.

Above we drew the simplest possible interpolations between the weak coupling and strong coupling behaviors. These simple interpolations are consistent with the values of all of the order parameters we discussed at weak and strong coupling, but several other rather natural phase diagrams may also be drawn. For instance, figures 8-11 do not apply to any theory that undergoes a phase transition as a function of the coupling at zero temperature. Such a phase transition is certainly possible, and could be inserted into any of the diagrams of figures 8-11 as a vertical line that divides Phase I into two regions, distinguished, presumably, by an order parameter unrelated to the Polyakov loop. A case where we know that this happens is when the infinite volume theory exhibits global symmetry breaking due to quantum effects (for example, the $d=4 \mathcal{N}=1 S U(N)$ SYM theory, in which a chiral $\mathbb{Z}_{2 N}$ symmetry is spontaneously broken to $\mathbb{Z}_{2}$ at infinite volume). Since we 


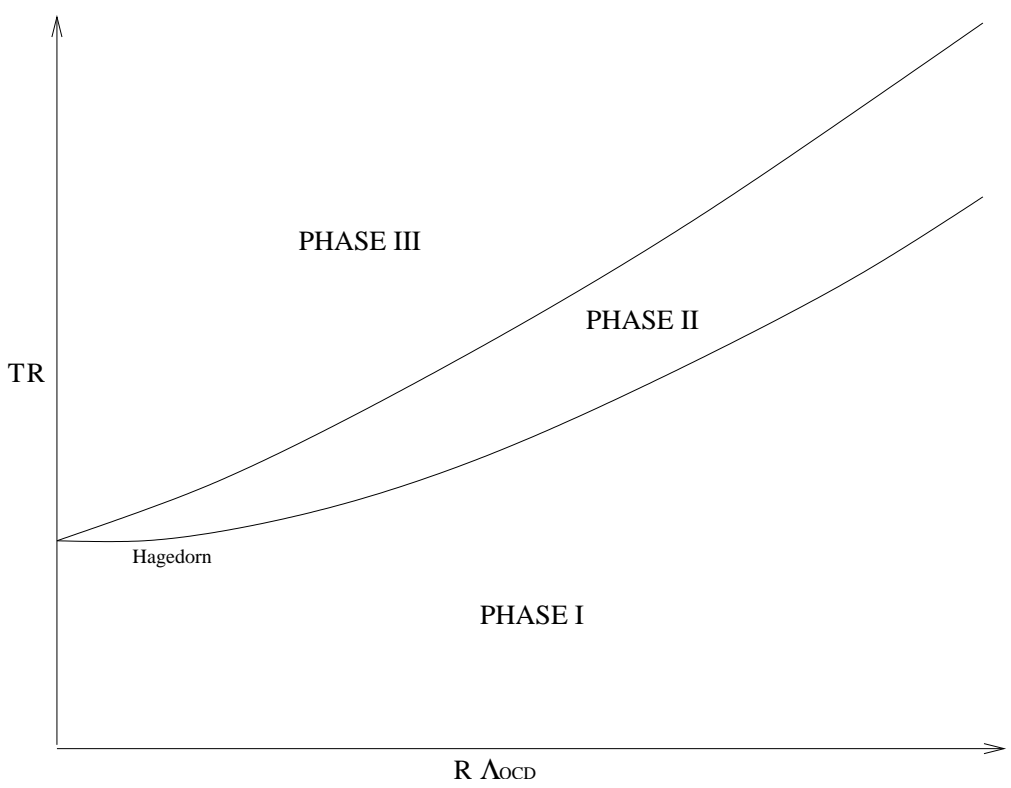

Figure 11: The simplest phase diagram for a compactified confining theory with positive $b$ and a second order phase transition at $R=\infty$.

see no sign of such a symmetry breaking in our analysis at weak coupling ${ }^{54}$, it seems clear that in such cases there are additional phase boundaries separating weak coupling and strong coupling regimes at low temperature. In general, we do not expect any such boundaries for asymptotically free gauge theories at high temperatures, since in this limit, the theories may be studied perturbatively for all values of $R \Lambda_{Q C D}$.

We now turn to the large $N S U(N) \mathcal{N}=4$ SYM theory on an $S^{3}$ of unit radius and at strong 't Hooft coupling $\lambda$. Using the AdS/CFT correspondence, this theory is equivalent to type IIB string theory at weak string coupling on an $A d S_{5} \times S^{5}$ space (using global coordinates for $A d S$ ) with a large radius of curvature. The thermodynamical analysis of this theory shows that it undergoes a single first order phase transition as a function of temperature, which occurs at $T_{H P}=\frac{3}{2 \pi} \simeq 0.477465[48,86]$. In addition, since the string coupling is small, we have a large range of energies with a Hagedorn behavior of the spectrum, with the Hagedorn temperature scaling as $T_{H} \propto 1 / \sqrt{\alpha^{\prime}} \propto \lambda^{1 / 4}$ and going to infinity in the limit of strong 't Hooft coupling.

\footnotetext{
${ }^{54}$ Of course, as in our discussion above of the breaking of the $\mathbb{Z}_{N}$ symmetry associated with confinement, we do not expect to see symmetry breaking at finite $N$ and finite volume, but we expect to see a sum over different configurations in which the symmetry is broken.
} 
On the other hand, as we have described in detail above, at $\lambda=0$ this theory also undergoes a single phase transition as a function of temperature. This phase transition, at the temperature $T_{H}=-1 / \ln (7-4 \sqrt{3}) \simeq 0.379663$, coincides with the Hagedorn transition and it is weakly of first order. And, as discussed in $\S 6$, the behavior of the theory at small $\lambda$ depends crucially on the sign of $b$ (defined in (6.7)). If $b$ turns out to be negative, the theory undergoes a single first order phase transition below the Hagedorn temperature. If $b>0$, the theory undergoes two continuous phase transitions, the first of which is at the Hagedorn temperature.

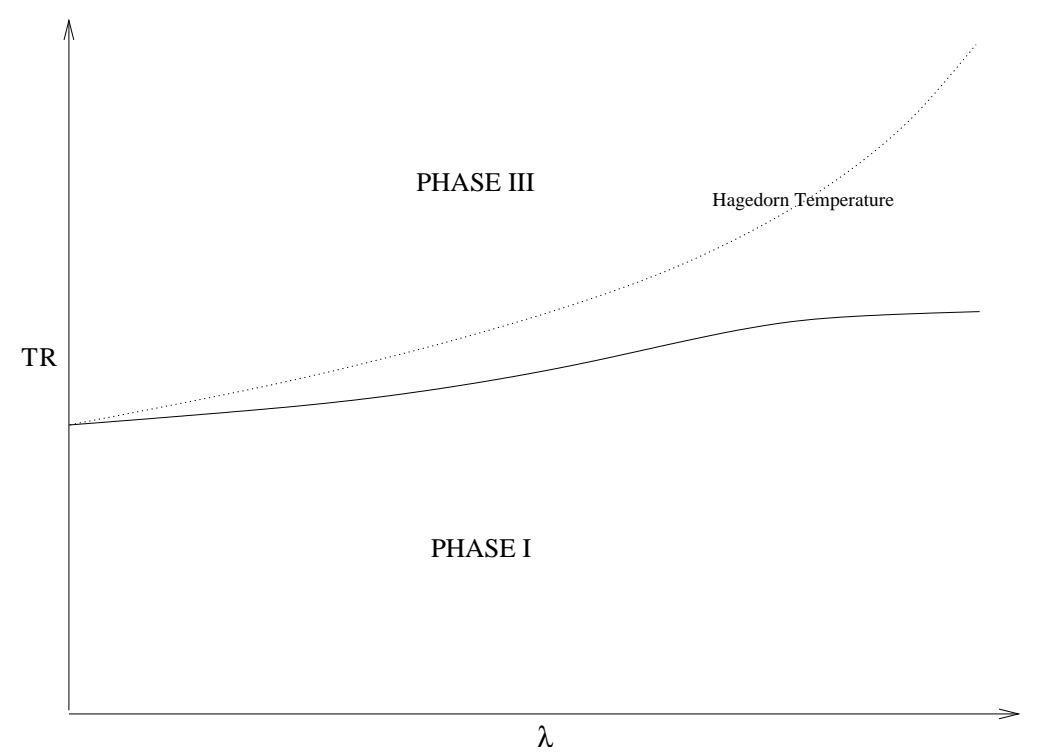

Figure 12: Conjectured phase diagram for the $\mathcal{N}=4$ SYM theory on a sphere if $b$ is negative. Only solid lines represent phase transitions.

As we have described above, the value of $b$ for this theory is determined by a set of two-loop and three-loop graphs. We will present the result of this computation in [3]. If $b$ turns out to be negative, the behaviors at weak and strong coupling are similar and the phase diagram of the theory has a natural interpolation (shown in figure 12) for all $\lambda$ (though more complicated phase diagrams are also possible).

On the other hand, if $b$ turns out to be positive, the simplest possible phase diagram takes the form shown in figure 13. Note the existence of a tri-critical point at a special value of the 't Hooft coupling, at which the deconfinement phase transition changes from being second order to being first order, and the existence of a new phase at weak coupling and intermediate 


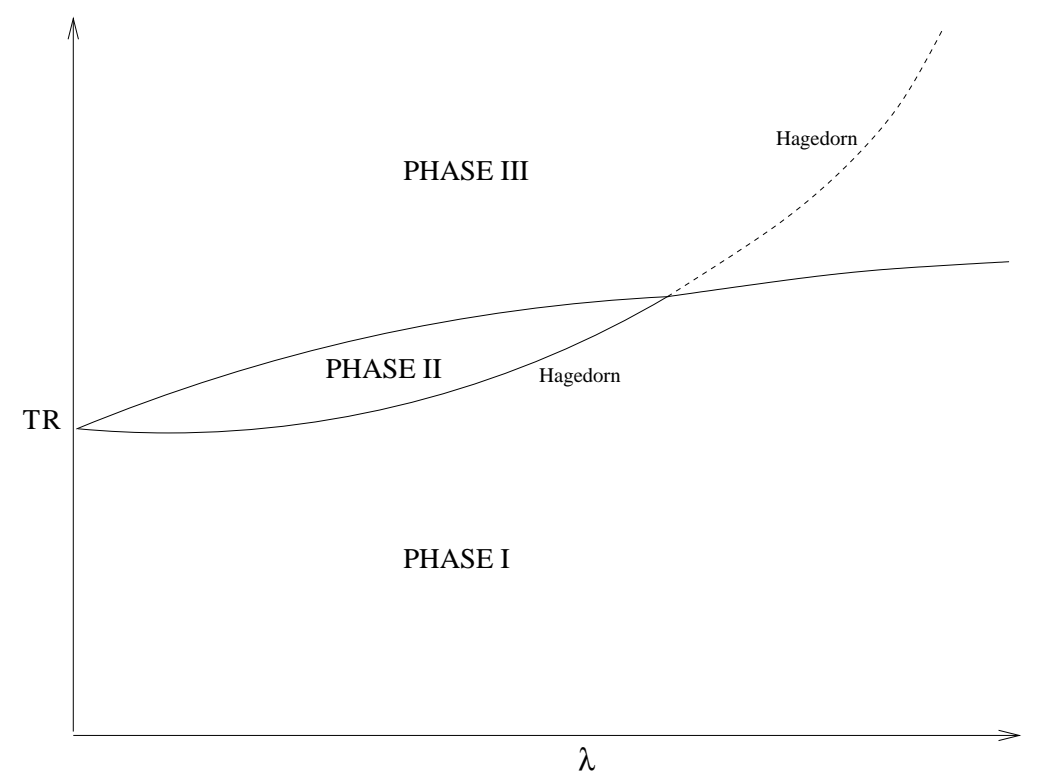

Figure 13: Conjectured phase diagram for the $\mathcal{N}=4$ SYM theory on a sphere if $b$ is positive. Only solid lines represent phase transitions.

temperatures. ${ }^{55}$

Figures 12 and 13 are very similar to figures 8 and 9, with one important difference: the phase transition temperature, measured in units of $1 / R$, has a finite strong coupling limit for the $\mathcal{N}=4$ theory, but increases without bound for confining theories.

Note that the interaction potential between a quark and an anti-quark, at a distance $L \ll R$, is expected to behave rather differently in the various phases described above. In the case of confining gauge theories at low temperatures, this potential will be Coulomb-like at weak coupling $(R \Lambda \ll 1)$ but linear (string-like) for $R \Lambda \gg 1$, as long as $L \gg 1 / \Lambda$. However, in Phase III (and presumably in Phase II as well) the quark-anti-quark force is exponentially screened by the intervening plasma. In the case of the $\mathcal{N}=4 \mathrm{SYM}$ theory the quark-anti-quark force is Coulomb-like at every coupling in the 'confining' Phase I, but decays exponentially at sufficiently large distances

\footnotetext{
${ }^{55}$ Figures 12 and 13 have been drawn under the assumption that the large $N \mathcal{N}=4$ theory in flat space does not undergo a phase transition as a function of the coupling. Such a transition could perhaps occur, and then it would be depicted by a vertical line that would divide Phase III into two different regions in figures 12 and 13 . We thank R. Gopakumar for emphasizing this possibility to us. Similarly, it is possible in principle that Phase I would be divided into different regions.
} 
(compared to a scale set by the temperature) in the deconfined phases.

\subsection{Dual interpretation of the $\mathcal{N}=4$ SYM thermodynamics at strong coupling}

We noted in $\S 2$ that it should be possible to understand the possible phase diagrams presented earlier in this section in terms of a dual string theory description of the relevant gauge theories. Unfortunately, among the $3+1$ dimensional gauge theories with adjoint fields, the only one whose string dual is known is the $\mathcal{N}=4$ supersymmetric Yang-Mills theory (and theories related to it by renormalization group flows), and this dual is mostly understood only in the limit of strong 't Hooft coupling. In this subsection we will argue that, at least in this one case, the thermodynamics of the string theory dual fits rather nicely with the picture presented in this paper. Previous discussions of the thermodynamics of $\mathcal{N}=4 \mathrm{SYM}$ as a function of the coupling, which are consistent with ours, appear in [11], and a detailed discussion of the transitions discussed in this subsection appears in $[13,9,10]$.

The string dual to $\mathcal{N}=4 \mathrm{SYM}$ on $S^{3} \times \mathbb{R}$ (where the $S^{3}$ is taken to be of unit radius) is type IIB string theory on $A d S_{5} \times S^{5}$, whose metric may be written as

$$
d s^{2}=R_{0}^{2}\left(-\cosh ^{2} \rho d \tau^{2}+d \rho^{2}+\sinh ^{2} \rho d \Omega_{3}^{2}+d \Omega_{5}^{2}\right) .
$$

Under this duality the Hamiltonian of the Yang-Mills theory is identified with the generator of global time translations $\partial_{\tau}$ in the geometry (7.1). The energy $E$ of the gauge theory on a sphere of unit radius is related to the proper energy in string theory at $\rho=0$ by $E_{\text {prop }}=E / R_{0}$. The radius $R_{0}$ of the $A d S_{5} \times S^{5}$ space is related to the 't Hooft coupling $\lambda$ and the inverse string tension $\alpha^{\prime}$ by $R_{0} \simeq \lambda^{\frac{1}{4}} \sqrt{\alpha^{\prime}}$ (we will ignore all numbers of order unity in the qualitative discussion of this subsection).

For large $R_{0} / \sqrt{\alpha^{\prime}}$ (large 't Hooft coupling) the density of states $\rho(E)$ of type IIB string theory on (7.1) has four distinct regimes (see [2] and references therein). The only states in the spectrum with proper energy ${ }^{56}$ smaller than the string scale, $E \ll \lambda^{1 / 4}$, are ten dimensional supergravitons ${ }^{57}$ whose entropy scales as $S(E) \propto E^{9 / 10}$. For $E \gg \lambda^{1 / 4}$, excited string states are added to the spectrum; the contribution of these states to the entropy

\footnotetext{
${ }^{56}$ Recall that states of finite energy on (7.1) are all localized about $\rho=0 . A d S_{5}$ space behaves effectively like a four dimensional box of physical radius $R_{0}$, as is apparent from the fact that the spectrum of $\partial_{\tau}$ is discrete with discretization step unity.

${ }^{57}$ In terms of the gauge theory, the only states in this regime are those created by the chiral primary operators, their products and their descendants.
} 
is $S \simeq E_{\text {prop }} \sqrt{\alpha^{\prime}} \simeq \lambda^{-1 / 4} E$ (see $\S 2.1$ ), and this contribution dominates over the graviton gas for $E \gg \lambda^{5 / 2}$. When the proper energy exceeds the Planck mass (namely, $E \gg m_{s} R_{0} / g_{s}^{1 / 4}=N^{1 / 4}$ ) small ten dimensional Schwarzschild black holes are also added to the spectrum (note that these energies are inaccessible in the strict $N \rightarrow \infty$ limit). The entropy of such black holes is proportional to $S \sim\left(l_{P} E / R_{0}\right)^{8 / 7}=\left(E / N^{1 / 4}\right)^{8 / 7}$; they have negative specific heat and positive free energy. This entropy of black holes dominates over that of the Hagedorn strings for $E \gg N^{2} / \lambda^{7 / 4}$. Finally, at an energy $E_{1} \sim N^{2}$ (an energy at which the radius of these Schwarzschild black holes becomes comparable to the $A d S$ radius $R_{0}$ ) the black hole horizon covers the whole $S^{5}$, and the specific heat of these black holes becomes positive. For $E>E_{1}$ these black holes are referred to as $A d S$-Schwarzschild or big black holes. At a higher energy $E_{2}$ (also of $\mathcal{O}\left(N^{2}\right)$ ) the free energy of these black holes becomes negative. Finally, for $E \gg N^{2}$, the entropy of these black holes is $S \propto N^{1 / 2} E^{3 / 4}$, so the thermodynamics of these big black holes resembles that of a four dimensional conformal field theory.

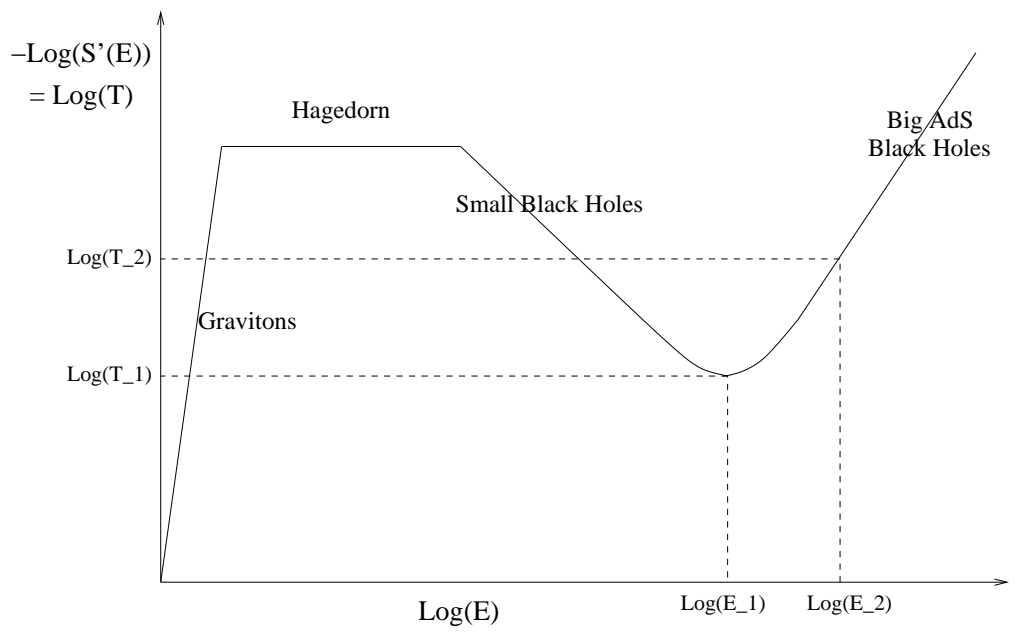

Figure 14: $\log (T)$ as a function of $\log (E)$ (in the microcanonical ensemble) for all the 'phases' of type IIB string theory on $A d S_{5} \times S^{5}$, when $R_{0} \gg \sqrt{\alpha^{\prime}}$.

For comparison with the canonical ensemble it is most convenient (see $\S 6.5)$ to display the density of states described in the previous paragraph as a plot (see figure 14) of $\ln (T(E))=-\ln \left(S^{\prime}(E)\right.$ ) [9]. Quite remarkably, figure 14 is identical in its general form to figure 7 , even though figure 7 was derived for a general gauge theory (with $b<0$ ) at weak coupling while figure 14 applies to the $\mathcal{N}=4$ Yang Mills theory at strong coupling, and has been derived using its stringy dual. 
To complete this subsection we now use figure 14 to discuss the behavior of the $\mathcal{N}=4 \mathrm{SYM}$ theory at strong coupling in the canonical ensemble [9] (see $\S 6.5$ for a very similar discussion). For $T<T_{1}\left(=\frac{\sqrt{2}}{\pi}\right)$ the only available saddle point is the thermal gas of gravitons. Over the temperature range $T_{1}<T<T_{H} \simeq \lambda^{\frac{1}{4}}$ the theory has three saddle points to choose from. One of these saddle points (small black holes) is unstable, so it cannot appear in the canonical ensemble. The other two saddle points are stable and compete with each other. The gas of gravitons has lower free energy for $T_{1}<T<T_{2}\left(=\frac{3}{2 \pi}\right)$, while the big black hole dominates the canonical ensemble for $T_{2}<T<T_{H}$. Consequently, the theory should undergo a first order phase transition at $T=T_{2}$, which (as we mentioned above) is indeed the case $[48,86] . T=T_{H}$ is the Hagedorn temperature at which the graviton gas saddle point stops existing. For $T>T_{H}$ the generic state of the theory continues to be a big black hole, which is now the only available phase.

\subsection{Deconfinement and black holes}

We do not, as yet, understand the string dual of weakly coupled gauge theories, such as pure Yang-Mills or $\mathcal{N}=1$ supersymmetric Yang-Mills on a small $S^{3}$, or even the $\mathcal{N}=4 \mathrm{SYM}$ theory at weak 't Hooft coupling. Nonetheless, each of these theories is continuously related to the $\mathcal{N}=4$ theory at strong coupling, so the AdS/CFT correspondence provides a demonstration (in the physicist's sense of the term) of the existence of string duals for all these gauge theories. Furthermore, these string duals may be expected to qualitatively resemble type IIB string theory on $A d S^{5} \times S^{5}$, in the sense that they should all include at least one additional "holographic" dimension beyond the dimensions of the Yang-Mills theory. Consequently, even though we understand little about the details of the string duals of arbitrary gauge theories, we will use intuition from the previous subsection to boldly speculate on the stringy dual interpretation of the phase diagrams in figures $8-13$.

As we reviewed above, the deconfined phase of the strongly coupled $\mathcal{N}=$ 4 theory admits a dual description in terms of black holes ${ }^{58}$. We would now like to argue that this is a general feature (see [77] for related comments): large $N$ deconfined phases (Phases II or III in figures 8-13) should always be

\footnotetext{
${ }^{58}$ The connection between deconfinement transitions and black holes has also been verified in examples of confining field theories in flat space, such as the duality between a cascading $d=4 S U(N+M) \times S U(N) \mathcal{N}=1$ supersymmetric gauge theory and type IIB string theory on a manifold which includes the resolved conifold [53, 17]. Similar results for the Maldacena-Nunez background [61] were obtained in [18], and for other theories in (for example) [31].
} 
associated with black holes ${ }^{59}$. Our simple argument follows directly from ideas presented in [86].

In order to measure the Polyakov loop we need to put an external quark on a trajectory that wraps around the circle of the time direction. Since in the large $N$ gauge/string correspondence quarks appear at the boundaries of the worldsheet, we expect the Polyakov loop in string theory to be realized as the partition function (in Euclidean space) for a string whose boundary wraps around the time-like loop, and this is indeed the case in the AdS/CFT correspondence $[59,75]^{60}$. This partition function clearly vanishes if the Euclidean time circle is non-contractible ${ }^{61}$, but it is generically non-zero (when suitably defined, as in our discussion of $\S 5.7$ and the analogous discussions in $[86,4])$ if the time circle is contractible. In all the examples that we know of, a static Euclidean time cycle is contractible in general relativity only when the corresponding Minkowskian solution has a horizon. Outside the range of validity of general relativity it would seem reasonable to define black holes by this requirement. Putting all this together, we conclude that suitably defined Polyakov loops are non-zero only in a black hole phase. Thus, we generally expect that the string theory (at finite temperature) in Phases II and III will be in a generalized black hole background, while the theory in Phase I (where the time cycle is non-contractible) should be in a background similar to the naive gauge theory background (in which the time direction is simply compactified).

A similar conclusion follows by analyzing the other order parameter for confinement, $\lim _{N \rightarrow \infty} F(T) / N^{2}$. In string theory this order parameter maps to the sphere partition function, which naively always vanishes. However, this is not true even in the low-energy general relativity approximation for some types of unbounded spaces $[48,86]$, where the naive classical action diverges and one needs a subtraction procedure to define it. This can lead to a non-zero result for the relative classical partition function between two spaces with the same asymptotics but a different interior. This again suggests that deconfined phases must involve different space-times than the

\footnotetext{
${ }^{59} \mathrm{By}$ this we mean that if the background has a geometrical interpretation it would resemble a black hole; we generalize this to other backgrounds by calling such phases "black holes".

${ }^{60}$ In this case the boundary of the string is actually not precisely the Polyakov loop, as it contains also couplings to scalar fields. We expect that these additional couplings will not change the qualitative features of the order parameter, and that these "generalized Polyakov loops" will vanish if and only if the usual Polyakov loop vanishes. Computations of such "generalized Polyakov loops" in the AdS/CFT correspondence first appeared in $[86,74,16]$.

${ }^{61}$ Again, this is true when we have a good geometrical description of the background, and otherwise we take the vanishing of the Polyakov loop to be the definition of a noncontractible circle.
} 
confined phase (which are the same asymptotically but differ in the interior), which have different classical actions. The discussion of the previous paragraph suggests that these spaces should be generalized black holes.

\subsection{Dual description at a general point in the phase diagram}

At any fixed value of the coupling in each of the diagrams of figures 8-13, the compactified large $N$ gauge theory either

(1) Undergoes a single first order phase transition as a function of the temperature away from a tri-critical point.

(2) Undergoes a single first order phase transition as a function of the temperature at a tri-critical point.

(3) Undergoes two successive continuous phase transitions as a function of the temperature.

It is possible (see below for a caveat) that the density of states takes the form shown in figures 7, 6 and 5, respectively, in every realization of the three cases listed above. We have already argued that this statement is true at weak coupling (see $\S 6.5$ ), and for the $\mathcal{N}=4$ SYM theory at strong coupling.

If this picture turns out to be correct, it would be tempting to further speculate that the dual description of Phase III always involves a stable 'big black hole'. Following this train of thought, one would also be led to speculate (following $\$ 7.2$ ) that Phase II has a dual description in terms of a different type of strange 'black holes' (see $\S 7.3$ for our usage of the term black holes). It would then follow that the discovery of theories of type (3) (theories with a second order large $N$ deconfinement phase transition) would imply the existence of a new class of stable 'strange black holes' in a class of string theories. This would certainly be intriguing; it would be very interesting to understand the string theory interpretation of the distinction between the two classes of 'black holes' (Phase II and Phase III).

Before concluding this section, we should note that the different theories and phases which we grouped together in our discussion above do seem to have some qualitative differences, despite sharing some similar features. Let us first study the behavior of a confining gauge theory (like $d=4$ pure Yang-Mills theory) as a function of coupling in more detail. On a very large compact space (in units set by $\Lambda_{Q C D}$ ) the pure Yang-Mills theory behaves locally just like the infinite volume theory, and we expect that the 
deconfinement transition proceeds (as energy is added to the system at the transition temperature) by nucleation of bubbles of the deconfined phase, which grow and eventually coalesce to cover the whole space. In particular, in this strong coupling limit there should be stable configurations at the transition temperature characterized by the coexistence of confined and deconfined phases covering different regions of the compact space. ${ }^{62}$ In such mixed configurations we would expect the size of every phase bubble to be at least of order $\Lambda_{Q C D}^{-1}$; in particular, mixed phases may be expected to be absent on a compact space that is small in QCD units, namely at weak coupling $\Lambda_{Q C D} R \ll 1{ }^{63}$ This is consistent with the analysis in our paper; recall that the order parameter we used for the phase transition is (roughly) the constant mode of $\left\langle\frac{1}{N} \operatorname{tr}(\mathrm{U}(\mathrm{y}))\right\rangle$ on the compact space, and that at weak coupling modes that describe inhomogeneous configurations of $\operatorname{tr}(\mathrm{U}(\mathrm{y}))$ are massive with a mass of the same order as the phase transition temperature, and may safely be integrated out, as we have done.

The situation is completely different in the case of the $d=4 \mathcal{N}=4$ SYM theory. In this theory the existence of a phase transition relies upon the compactness of the space at all values of the coupling, and the behavior at weak coupling and at strong coupling seem similar. Recall that, in the strongly coupled $\mathcal{N}=4$ theory, the dual description of the high temperature phase is a single large black hole. Since there would seem to be no such thing as half of a black hole, the high temperature phase cannot coexist with a low temperature phase at the transition temperature at strong coupling.

The contrasting behaviors of the systems described in the previous two paragraphs suggest that the "large black hole" phase (Phase III) of strongly coupled pure Yang-Mills theory may be significantly different from the large AdS black hole. It would be interesting to understand what sort of bulk description the coexistence of confined and deconfined phases in confining theories such as the pure Yang-Mills theory could have. One is reminded of the appearance of non-homogeneous configurations in the context of the Gregory-Laflamme transition [38].

\footnotetext{
${ }^{62}$ Note that in our discussion above of microcanonical phase diagrams (and, indeed, in our whole analysis which was based on the effective action $S_{\text {eff }}(U)$ ), we were implicitly discussing only homogeneous phases. It seems that at least in some cases non-homogeneous configurations dominate at strong coupling (in the microcanonical ensemble) over the configurations we described above.

${ }^{63}$ The fact that the qualitative behavior of Yang-Mills theories changes in this respect in the transition from weak coupling to strong coupling may indicate that the naive interpolation we suggested between these two regimes is too optimistic.
} 


\section{Discussion and future directions}

In this paper we have analyzed the thermodynamics of weakly coupled large $N$ gauge theories compactified on a sphere of radius $R$, or any other compact manifold on which the theory has no zero modes. Our analysis applies both to conformal gauge theories at small values of a tunable coupling constant (such as the $\mathcal{N}=4 \mathrm{SYM}$ theory) and to confining theories (such as pure or $\mathcal{N}=1$ Yang-Mills theory) with $R \Lambda_{Q C D} \ll 1$. We have shown that in the microcanonical ensemble these theories exhibit an exponential (Hagedorn) density of states, which is cut off at an energy of order $E \sim N^{2}$ (this was previously shown for free gauge theories in $[77,73])$. The Hagedorn temperature at zero coupling is easily determined from the field content of the theory, and the corrections to it can be computed in perturbation theory. We have demonstrated that these theories undergo deconfinement phase transitions, with different possible phase diagrams discussed in $\S 6$.

Our analysis has several points of interest. First, putting a confining gauge theory on a compact space introduces a new dimensionless parameter into the game; varying this parameter may continuously deform the flat space deconfinement transition into a regime where the transition (which remains non-trivial) may be reliably studied in perturbation theory ${ }^{64}$. The analysis of this weakly coupled problem leads to several insights. For instance, we have argued that if the large $N$ deconfining phase transition is of second order then it must be Hagedorn-like (as first shown from different arguments in [67]), and it must be followed by a second phase transition at a higher temperature (where the eigenvalue distribution corresponding to a holonomy around the thermal circle develops a gap). This suggests that a second order deconfining transition in any large $N$ gauge theory implies the existence of a previously unsuspected intermediate temperature phase. Our analysis of the weak coupling deconfinement transition led to us suggest the four simplest possible phase diagrams (figures 8-11) for confining large $N$ gauge theories; which diagram is actually implemented depends on the details of the theory, including the sign of a perturbatively computable number. More speculatively, we have also suggested a stringy interpretation (in terms of nucleation of black holes in the dual string theory) of this deconfinement transition.

Turning now to the $\mathcal{N}=4$ supersymmetric Yang-Mills theory, our results imply that the thermal phase transition in this theory at weak coupling is

\footnotetext{
${ }^{64}$ This is somewhat analogous to the continuous relation between confining phases and perturbatively-accessible Higgs phases which can occur [8] in theories with fields in the fundamental representation upon changing coupling constants or vacuum expectation values of fields.
} 
either of first or second order, depending on the sign of a coefficient that we are now in the process of computing. If the transition turns out to be of first order, the simplest possible conclusion would be that the Hagedorn spectrum of string theory on $A d S_{5} \times S^{5}$ does not dominate the thermodynamics of $\mathcal{N}=$ $4 \mathrm{SYM}$ at any temperature and at any non-zero value of the coupling - this was called "Hagedorn censorship" in [11]. In such a case our computations would enable us to study an unstable saddle point representing substringy unstable ('Schwarzschild') black holes in this background. On the other hand, if the weak coupling transition turns out to be of second order, that would suggest the existence of a tri-critical point at some finite 't Hooft coupling (probably of order one) in this supersymmetric Yang-Mills theory, as well as the existence of a previously unsuspected intermediate temperature phase in this theory at weak coupling. This new intermediate temperature phase should then have a dual description in terms of a new set of bulk objects: mysterious new stable black holes.

The picture we present in this paper supports the view that 't Hooft's relation between large $N$ gauge theories and string theories [80] may extend also to weakly coupled gauge theories. The naive picture of this relation is that at strong coupling the sum over planar Feynman diagrams is dominated by graphs with arbitrarily many interactions, which become dense and close up the holes in the worldsheet of the Feynman diagram to form a continuous $1+1$ dimensional field theory on a spherical Riemann surface. This view has already been seriously challenged by the AdS/CFT correspondence, which establishes a relationship between string theories and gauge theories on a sphere at all values of the gauge coupling ${ }^{65}$, and by the consequent derivation of dualities between (topological) open and closed string theories [37, 65]. Our findings provide further evidence for the existence of a stringy dual of weakly coupled gauge theories. We have found that weakly coupled gauge theories on compact manifolds share at least one qualitative feature of string theory in flat space, namely a string-like spectrum, in a regime in which the Feynman diagrams do not seem to look like continuous Riemann surfaces. It would be very interesting to understand more directly how and why weakly coupled gauge theories manage to rearrange themselves as string theories (see $[45,36,12]$ for some attempts in this direction).

It is an interesting challenge to identify the string theories which are dual to various weakly coupled (or free) gauge theories. For the free gauge

\footnotetext{
${ }^{65}$ Although the worldsheet theories dual to perturbative gauge theories are strongly coupled, as a consequence of large background curvatures, they presumably exist, as they may be obtained from the well-understood duals to strongly coupled gauge theories by tuning worldsheet parameters. See $[85,51,70,28,23,14,27]$ for recent attempts to understand this weakly coupled limit.
} 
theories our analysis of $\S 3$ gives us an explicit formula (3.6) for the spectrum of (free) single-string states, and perhaps in some cases this may be enough to reconstruct the corresponding worldsheet theory. By expanding our result for the partition function in a power series in $1 / N^{2}$, we can also extract information about string coupling corrections in the putative dual string theory (though it may turn out that real string interactions always involve the Yang-Mills interaction, as in the study of string interactions on plane waves [25]).

The results of the computations in this paper (and our upcoming work [3]) may turn out to have independent interest. For conformal theories such as the $d=4 \mathcal{N}=4$ supersymmetric Yang-Mills theory, the partition function computed in this paper and in [3] encodes, in principle, the scaling dimension of every operator in the theory, to the order of computation (in $\lambda)^{66}$. The information about these anomalous dimensions is packaged in an interesting fashion in $S_{\text {eff }}$ and may lead to insights, perhaps in combination with recent speculations on integrability and on the existence of a large Yangian symmetry in the $\mathcal{N}=4$ SYM theory [63].

The techniques of this paper may find application to several theories not explicitly considered in this paper. It would be interesting to compare our results to known results in strongly coupled two dimensional gauge theories $[83,54,39]$. On another note, recall that large $N$ gauge theories in $d=1$ with a single scalar field are believed to be holographically dual to the $c=1$ and $\widehat{c}=1$ string theories. Our analysis of free one-matrix positive-sign harmonic oscillators at zero coupling shows that they have neither phase transitions nor a Hagedorn-like spectrum; however, the matrix models dual to the $c=1$ and $\widehat{c}=1$ strings are 'negative mass' harmonic oscillators, and it is possible that they somehow undergo a 'deconfining transition' of the sort studied in this paper, described by some effective action $S_{\text {eff }}\left(u_{1}\right)$, and that their high energy behavior is dominated by two dimensional black holes (whose spectrum is Hagedorn-like). ${ }^{67}$ Another interesting example is provided by the 't Hooft limit of the maximally supersymmetric plane-wave deformation of Matrix theory, argued in [62] to be dual to a little string theory compactified on $S^{5}$. For a small sphere, this theory is weakly coupled, and one may study the little string theory thermodynamics explicitly in this limit using methods similar to this paper. This analysis is the subject of a paper [32] to appear shortly. Another interesting relation between the thermodynamics of string theory, black holes and the thermodynamics of compactified gauge theories appears in the context of Matrix theory (see, for instance, [57]). It

\footnotetext{
${ }^{66}$ This is true at least in the absence of first order phase transitions.

${ }^{67}$ Several people, including A. Adams, P. Ho, G. Mandal, and S. Wadia, have suggested a variant of this idea.
} 
would be interesting to try to relate our discussion to the results for toroidal compactifications which are relevant there. When we compactify a gauge theory on a space with a non-zero fundamental group, we necessarily have additional zero modes coming from the non-trivial Wilson loops. It would be interesting to generalize our results to include such additional degrees of freedom. Some recent results on the deconfinement transition of the large $N$ gauge theory on a torus in the strong coupling limit appear in [64].

We should also note that the connection between Hagedorn transitions, black hole formation and some sort of 'deconfinement' transition in a dual field theory seems more general than the specific example of gauge theories. For instance, string theory on $A d S_{3}$ is known to undergo a phase transition as a function of temperature both at 'weak coupling' [26] (at the orbifold point) and at strong coupling (in a geometric phase for the bulk). At weak coupling this transition (between a single "long string" phase and a multiple "short string" phase) is Hagedorn-like [29], while at strong coupling it is a first order Hawking Page transition [48] of the sort reviewed in this paper. It would be interesting to analyze the extrapolation between these two behaviors.

Finally, several technical and conceptual issues remain to be addressed even within the direct line of attack of our paper. The coefficient $b$ should certainly be computed for several gauge theories [3]. The analysis of this paper can be extended to search for interesting features in generalized partition functions ${ }^{68}$. And, last but not least, the dual interpretation of deconfining phase transitions and their intriguing connection with black holes certainly deserves further study.

\section{Acknowledgements}

We would like to thank T. Banks, R. Dijkgraaf, A. Das, S. Elitzur, D. Gross, M. Headrick, C. Johnson, S. Kachru, I. Klebanov, J. Maldacena, G. Mandal, L. Motl, A. Neitzke, B. Pioline, J. Polchinski, E. Rabinovici, A. Schwimmer, A. Sen, T. Senthil, S. Sethi, Y. Shamir, S. Shenker, A. Stro-

\footnotetext{
${ }^{68}$ For instance, the partition function generalized by the addition of a chemical potential for an $R$-symmetry charge in the strongly coupled $\mathcal{N}=4 \mathrm{SYM}$ theory undergoes a phase transition as a function of the chemical potential even at zero temperature [22, 19]. By generalizing our analysis of this paper to this case, using the results of $\S 3.4$, we find that this zero temperature phase transition is absent in the free Yang-Mills theory. Note that for finite temperature our analysis breaks down at a critical value of the chemical potential when additional degrees of freedom become light and charged scalars may condense [42]. So (as noted for the compactified gauge theory in [49]) the strong coupling and weak coupling regimes seem qualitatively different in this case. Similar considerations may allow us to make contact with string thermodynamics on plane waves [66].
} 
minger, T. Takayanagi, W. Taylor, A. Vishwanath, A. Yarom, X. Yin and especially N. Arkani Hamed, R. Gopakumar, G. Semenoff, and S. Wadia for useful discussions. We would like to thank Stanford University and the Stanford-Weizmann workshop (partly financed by the Israel-U.S. Binational Science Foundation) for hospitality during the genesis of this project. OA would like to thank the University of Chicago, the second Crete regional meeting on String Theory, the Strings 2003 conference in Kyoto, the Aspen Center for Physics, and the University of British Columbia for hospitality during the work on this project. JM would like to thank PIMS and UBC for hospitality during the FMP school. SM would like to thank the Tata Institute of Fundamental Research, ICTP Trieste, the organizers of the regional summer school at Villa de Levya, Columbia, the second Crete regional meeting on String Theory, the Benasque post-Strings workshop, and the Indian Institute for the Cultivation of Sciences for hospitality while this work was in progress. MVR would like to thank the Korean Institute for Advanced Study and the Asia Pacific Center for Theoretical Physics for hospitality while this work was in progress. The work of OA was supported in part by the Israel-U.S. Binational Science Foundation, by the ISF Centers of Excellence program, by the European network HPRN-CT-2000-00122, and by Minerva. OA is the incumbent of the Joseph and Celia Reskin career development chair. The work of JM was supported in part by an NSF Graduate Research Fellowship. The work of SM was supported in part by DOE grant DE-FG01-91ER40654 and a Harvard Junior Fellowship. The work of KP was supported in part by DOE grant DE-FG01-91ER40654. The work of MVR was supported in part by NSF grant PHY-9870115, by funds from the Stanford Institute for Theoretical Physics, by NSERC grant 22R81136 and by the Canada Research Chairs programme.

\section{A Properties of group characters}

In applications of group theory, it is often necessary to determine the set of irreducible representations obtained in the tensor product of some collection of other representations $R_{i}$. Characters provide a powerful tool to achieve this. Given a group $G$, we may associate with any representation $R_{i}$ a character $\chi_{R_{i}}: G \rightarrow \mathbb{C}$, defined such that $\chi_{R_{i}}(U)$ is equal to the trace of the group element $U$ in the representation $R_{i}$. From this definition, it follows that the characters for sums and products of representations are given by

$$
\chi_{R_{1} \oplus R_{2}}=\chi_{R_{1}}+\chi_{R_{2}}, \quad \chi_{R_{1} \otimes R_{2}}=\chi_{R_{1}} \cdot \chi_{R_{2}} .
$$


The utility of the characters in decomposing tensor products follows from the orthogonality of characters for irreducible representations $R_{i}^{I}$ :

$$
\int[d U] \chi_{R_{1}^{I}}^{*}(U) \chi_{R_{2}^{I}}(U)=\delta_{R_{1}^{I} R_{2}^{I}}
$$

where $[d U]$ is the invariant (Haar) measure on the group manifold normalized so that $\int[d U]=1$. Thus, the number of irreducible representations of type $R^{I}$ in the tensor product of the representations $R_{1}, \ldots, R_{n}$ is given by

$$
n_{R^{I}}=\int[d U] \chi_{R^{I}}^{*}(U) \prod_{i=1}^{n} \chi_{R_{i}}(U) .
$$

For the special case of the trivial (singlet) representation, we have $\chi_{\text {singlet }}(U)$ $=1$, so that the number of singlets in the product of representations $R_{i}$ is simply

$$
n_{\text {singlet }}=\int[d U] \prod_{i} \chi_{R_{i}}(U) .
$$

Finally, for the applications of this paper, we require the character formulae for the symmetrized and antisymmetrized products of $n$ identical representations $R$. To obtain these, let $U_{R}$ be the matrix representation of the group element $U$ in the representation $R$. Then the trace of the matrix representation of $U$ in the (anti)symmetrized tensor product of $n$ copies of $R$ is

$$
\left(U_{R}\right)_{\left[a_{1}\right.}^{a_{1}} \cdots\left(U_{R}\right)_{\left.a_{n}\right]_{ \pm}}^{a_{n}}
$$

where the $[\cdots]_{ \pm}$indicates symmetrization or anti-symmetrization of the indices with unit weight. This expression is exactly the $t^{n}$ term in the expansion of the integral

$$
G_{ \pm}(U, t)=\frac{1}{\pi^{\operatorname{dim}(R)}} \int\left[d \phi_{ \pm}\right] e^{-\bar{\phi}_{ \pm} \phi_{ \pm} \pm t \bar{\phi}_{ \pm} U_{R} \phi_{ \pm}}
$$

in powers of $t$, where $\phi_{+}$and $\phi_{-}$are complex bosonic or fermionic variables, respectively, in the representation $R$. Thus, the result of this integral

$$
G_{ \pm}(U, t)=\left(\operatorname{det}\left(1 \mp t U_{R}\right)\right)^{\mp 1}
$$

serves as a generating function for the characters of (anti)symmetrized products of arbitrary copies of the representation R. More explicitly, by expanding the determinant we may express the results directly in terms of the character for the representation $R$ as

$$
\begin{aligned}
& G_{+}(U, t) \equiv \sum_{n=0}^{\infty} t^{n} \chi_{\operatorname{sym}^{n}(R)}(U)=e^{\sum_{l=1}^{\infty} t^{l} \chi_{R}\left(U^{l}\right) / l}, \\
& G_{-}(U, t) \equiv \sum_{n=0}^{\infty} t^{n} \chi_{\operatorname{antin}^{n}(R)}(U)=e^{\sum_{l=1}^{\infty}(-1)^{l+1} t^{l} \chi_{R}\left(U^{l}\right) / l} .
\end{aligned}
$$




\section{B Counting states in $U(N)$ gauge theories}

In this appendix we derive the precise formula (3.6) for the counting of gauge-invariant states in a large $N$ theory with adjoint fields, and we discuss the single-particle partition functions for theories on a sphere with various field contents.

\section{B.1 Counting gauge-invariant states precisely}

In order to count the number of independent operators corresponding to traces of products of fields in the large $N$ limit, we wish to count the number of different arrangements of objects subject to an identification of arrangements related by a cyclic permutation. This can be done using Polya's theorem $[77,73]$.

Consider a set of $m$ types of objects, and associate a weight $x_{i}$ with each of these objects. The weight associated with a collection of these objects is simply the product of the weights associated with each of the individual objects. Polya solved the general problem of summing over weights for all sets of $k$ of these objects, two sets being treated as identical if they are related to each other under the action of a specified subgroup of the permutation group. The subgroup relevant to us is simply the cyclic subgroup of order $k$; we will state Polya's result for this case. Define the polynomial

$$
p_{k}\left(y_{1}, y_{2}, \ldots, y_{k}\right)=\frac{1}{k} \sum_{\pi} y_{1}^{n(\pi)_{1}} y_{2}^{n(\pi)_{2}} \ldots y_{k}^{n(\pi)_{k}}
$$

where the summation in (B.1) runs over all elements $\pi$ of the cyclic subgroup, and $n(\pi)_{i}$ is the number of cycles of length $i$ in the permutation $\pi$. The answer to the question addressed earlier in this paragraph is simply

$$
p_{k}\left(\sum_{i=1}^{m} x_{i}, \sum_{i=1}^{m} x_{i}^{2}, \ldots, \sum_{i=1}^{m} x_{i}^{k}\right) \text {. }
$$

Applying this result to our problem, we find that the large $N$ partition function of single-trace states with $k$ oscillators is precisely given by

$$
Z_{k}=p_{k}\left(z(x), z\left(x^{2}\right), z\left(x^{3}\right), \cdots, z\left(x^{k}\right)\right)
$$

where, as in $\S 3, z(x)$ is the single-particle partition function. This implies 
that

$$
\begin{aligned}
Z_{S T} & =\sum_{k=1}^{\infty} p_{k}\left(z(x), z\left(x^{2}\right), z\left(x^{3}\right), \cdots, z\left(x^{k}\right)\right) \\
& =\sum_{k=1}^{\infty} \frac{1}{k} \sum_{l=1}^{k} z(x)^{n(k, l)_{1}} z\left(x^{2}\right)^{n(k, l)_{2}} \cdots z\left(x^{k}\right)^{n(k, l)_{k}},
\end{aligned}
$$

where $n(k, l)_{q}$ refers to the number of cycles of length $q$ in the cyclic permutation by $l$ shifts of $k$ objects.

It is easy to convince oneself that for specific values of $k$ and $l, n(k, l)_{q}$ is non-zero for only one value of $q$. At that value of $q$ it is given by $G(l, k)$, the greatest common divisor of $l$ and $k$. It then follows that the $q$ for which $n(k, l)_{q}$ is non-zero is given by $q=k / G(l, k)$. Consequently, (B.3) may be rewritten as

$$
Z_{S T}=\sum_{k=1}^{\infty} \frac{1}{k} \sum_{l=1}^{k} z\left(x^{k / G(l, k)}\right)^{G(l, k)} .
$$

We now group together all terms with the same (fixed) $q=k / G(l, k)$, so that $k=G(l, k) q$. Denoting $j=G(l, k)$, we change the sum over $l$ and $k$ to a sum over $j$ and $q$, where each term appears once for every $l \leq j q$ such that $G(l, j q)=j$. The number of such $l$ 's is precisely $\varphi(q)$, the number of positive integers which are not larger than $q$ and are relatively prime to $q$. Thus, we obtain

$$
Z_{S T}=\sum_{j=1}^{\infty} \sum_{q=1}^{\infty} \frac{\varphi(q)}{j q} z\left(x^{q}\right)^{j}=-\sum_{q=1}^{\infty} \frac{\varphi(q)}{q} \ln \left(1-z\left(x^{q}\right)\right),
$$

as in (3.6).

\section{B.2 Evaluating single-particle partition functions on spheres}

Next, we turn to a different topic which is the evaluation of the single-particle partition functions for $d$-dimensional field theories compactified on $S^{d-1} \times \mathbb{R}$, with unit radius for $S^{d-1}$. This may be carried out directly by noting that the Laplacian on the sphere (or the spatial parts of the other wave operators corresponding to particles with spin) may be written directly in terms of angular momentum generators, which may be diagonalized in the usual way.

Alternatively, since free field theories are conformally invariant, and we are interested in conformally coupled fields (though it is easy to generalize our results also to other cases), we can use the conformal transformation 
that relates $S^{d-1} \times \mathbb{R}$ to $\mathbb{R}^{d}$. This transformation takes states of the field theory on $S^{d-1} \times \mathbb{R}$ to local operators on $\mathbb{R}^{d}$, with the energy of the state becoming the scaling dimension of the operator. Thus, an equivalent way to define the partition function in such a case is by $z(x)=\sum_{\text {local operators }} x^{\Delta}$, where $\Delta$ is the scaling dimension of the operator.

We begin by considering a free scalar field $\varphi$. The local operators in the theory are $\varphi, \partial_{i} \varphi, \partial_{i} \partial_{j} \varphi$, and so on, modulo the equation of motion. Ignoring the equation of motion for a moment, these operators are all generated by repeated application of the $d$ different derivative operators $\partial_{1}, \partial_{2} \ldots \partial_{d}$, each of which is of unit dimension and so has the partition function $\frac{1}{(1-x)}$, on the free field $\varphi$ of dimension $(d / 2-1)$. Multiplying the various partition functions we find

$$
z_{S}^{\prime}(x)=\frac{x^{d / 2-1}}{(1-x)^{d}}
$$

In order to obtain $z_{S}(x)$ we must subtract from this the partition function for operators that vanish by the equation of motion $\partial^{2} \varphi=0$. Such operators are generated by acting with an arbitrary number of derivatives on $\partial^{2} \varphi$, so their partition function is $x^{2} z_{S}^{\prime}(x)$. Thus, we find

$$
z_{S}(x)=\left(1-x^{2}\right) z_{S}^{\prime}(x)=\frac{x^{\frac{d}{2}}+x^{\frac{d}{2}-1}}{(1-x)^{d-1}} .
$$

As a check, we note that in $d=4$ the operators that we get by acting with $k$ derivatives are in the $k^{t h}$ traceless symmetric representation of $S O(4)$ which has $j_{1}=j_{2}=\frac{k}{2}$, and they have dimension $\Delta=k+1$. These are simply the scalar spherical harmonics on $S^{3}$. This implies that the number of operators of dimension $\Delta$ is $n_{S}(\Delta)=\left(2 j_{1}+1\right)\left(2 j_{2}+1\right)=\Delta^{2}$, consistent with the Taylor expansion of (B.8) for $d=4$.

Next, we turn to the free vector field. The number of gauge-invariant operators is independent of the gauge, so we can fix an arbitrary gauge for the counting. We will use the gauge $A_{0}=0$ on $S^{d-1} \times \mathbb{R}$, which becomes the gauge $x^{\mu} A_{\mu}=0$ after the conformal transformation to $\mathbb{R}^{d}$ (recall that, according to the state-operator map, all operators are to be evaluated at $x=0$ ). Differentiating the gauge condition at the point $x=0$ we find the relations

$$
A_{\mu}=0, \quad \partial_{\mu} A_{\nu}+\partial_{\nu} A_{\mu}=0, \cdots, \partial_{\left\{i_{1}\right.} \partial_{i_{2}} \ldots \partial_{i_{n}} A_{\left.i_{n+1}\right\}}=0, \cdots
$$

where the brackets \{\} denote symmetrization. To start with we ignore both (B.9) and the equation of motion - this leads to a single-particle partition function $z_{V}^{\prime}(x)=x^{2-\frac{d}{2}} d z_{S}^{\prime}(x)$ (since the gauge field must have scaling dimension one in any space-time dimension). Operators of dimension $\Delta$ that are 
set to zero by (B.9) are given by symmetric tensors of rank $\Delta$; based on the previous paragraph the corresponding partition function is $x^{1-\frac{d}{2}} z_{S}^{\prime}(x)-1$, where the last subtraction comes because there are no tensors of rank zero in (B.9). With the condition (B.9), the Maxwell equation (at $x=0$ ) simply reduces to $\partial^{2} A_{\mu}=0$. The number of independent operators set to zero by the equation of motion is, therefore, counted by $d x^{4-\frac{d}{2}} z_{S}^{\prime}(x)$. Finally, the number of operators set to zero by both the constraint (B.9) and the equation of motion is encoded in the partition function $x^{5-\frac{d}{2}} z_{S}^{\prime}(x)$. Putting it all together, using (B.7), we find

$$
\begin{aligned}
z_{V}(x) & =\frac{d x}{(1-x)^{d}}-\frac{1}{(1-x)^{d}}-\frac{d x^{3}}{(1-x)^{d}}+\frac{x^{4}}{(1-x)^{d}}+1 \\
& =1-\frac{(1+x)\left(1+x^{2}-d x\right)}{(1-x)^{d-1}} .
\end{aligned}
$$

As a check, we note that in four dimensions, the set of operators formed by acting with $k$ derivatives on $A_{\mu}$, obeying (B.9) and the equation of motion, transform in the $S O(4)$ representation $\left(j_{1}, j_{2}\right)=\left(\frac{k+1}{2}, \frac{k-1}{2}\right) \oplus\left(\frac{k-1}{2}, \frac{k+1}{2}\right)$. These are the vector spherical harmonics on $S^{3}$. It follows that the number of operators at dimension $\Delta$ is $n_{V}(\Delta)=2\left(\Delta^{2}-1\right)$, consistent with (B.10).

Finally, we turn to free fermions. For concreteness we work in even dimensions with complex spinors of no chirality restrictions. Such a spinor has $2^{\frac{d}{2}+1}$ real components. Ignoring the equation of motion, the partition function for spinors is $z_{F}^{\prime}(x)=2^{\frac{d}{2}+1} \sqrt{x} z_{S}^{\prime}(x)$. The partition function that counts the operators which are set to zero by the Dirac equation, is $2^{\frac{d}{2}+1} x^{\frac{3}{2}} z_{S}^{\prime}(x)$. Subtracting the second from the first we find

$$
z_{F}(x)=\frac{2^{\frac{d}{2}+1} x^{\frac{d}{2}-\frac{1}{2}}}{(1-x)^{d-1}} .
$$

Of course, (B.11) should be divided by two for chiral spinors or real spinors, and by four for spinors that are both chiral and real. As a check on (B.11), note that, in $d=4$, the operators made from a complex chiral fermion field, at dimension $k+\frac{1}{2}$, transform in the $S O(4)$ representation with $\left(j_{1}, j_{2}\right)=$ $\left(\frac{k}{2}, \frac{k-1}{2}\right)$; there are $2 k(k+1)$ such operators (the factor of 2 is because the spinors are complex), in agreement with (B.11).

Note that each of (B.8), (B.10), and (B.11) tends as $x \rightarrow 1$ (the high temperature limit) to

$$
z(x) \rightarrow \frac{2 \mathcal{N}^{d o f}}{(1-x)^{d-1}},
$$

where $\mathcal{N}^{\text {dof }}$ is the number of physical real degrees of freedom in the corresponding field. 
The formulas in this section, used for $d=4$, imply the formula (3.20) of $\S 3$.

\section{Hagedorn transitions at finite string coupling}

There have long been speculations that at finite values of the string coupling $g_{s}$, one could have a phase transition at (or near) the Hagedorn temperature, with a different description of the theory at high temperatures. In this appendix we analyze, following [6], the effect of turning on a small string coupling $g_{s}$ on the partition function. Recall that, as reviewed in $\S 2.1$, the Euclidean partition function includes a winding mode $W$ that becomes tachyonic above the Hagedorn temperature. On general grounds, the perturbative effective action that describes the interaction between $W$ and all other modes (let us call them $\varphi_{n}$ ) takes the form

$$
\begin{aligned}
S_{e f f}= & \int d^{d} x\left(|\partial W|^{2}+\frac{2}{\alpha^{\prime}}\left(\frac{1}{8 \pi^{2} \alpha^{\prime} T^{2}}-1\right)|W|^{2}\right. \\
& \left.+\sum_{n} \frac{1}{2}\left(\partial \varphi_{n}\right)^{2}+\frac{M_{n}^{2}}{2} \varphi_{n}^{2}+\cdots\right) \\
& +\left(g_{s} \sum_{n} I_{n} \varphi_{n}|W|^{2}+g_{s}^{2} \theta|W|^{4}+\cdots\right) .
\end{aligned}
$$

If all other fields $\varphi_{n}$ are massive we can integrate them out, and remain with an effective action for $W$ near the Hagedorn temperature. After rescaling $W$ this takes the form

$$
S_{e f f}=\frac{1}{g_{s}^{2}} \int d^{d} x\left(|\partial W|^{2}+\frac{2}{\alpha^{\prime}}\left(\frac{1}{8 \pi^{2} \alpha^{\prime} T^{2}}-1\right)|W|^{2}+b|W|^{4}+\cdots\right)+\cdots,
$$

where

$$
b=\theta-\sum_{n} \frac{I_{n}^{2}}{2 M_{n}^{2}} .
$$

The dynamics of this theory depends crucially on the sign of $b$. For $b>0, S_{\text {eff }}$ is the Landau Ginzburg free energy for a system that undergoes a second order phase transition at $T=T_{H}$. In this case, for $T<T_{H}, S_{\text {eff }}$ is minimized at $W=0$. The 'saddle point' contribution to the free energy vanishes and the leading $\mathcal{O}\left(g_{s}^{0}\right)$ contribution to $\ln (Z)$ is given by the free string theory partition function. However, at temperatures just above $T_{H}$, $S_{\text {eff }}$ is minimized at

$$
|W|^{2}=\frac{T-T_{H}}{4 \pi^{2} b \alpha^{\prime 2} T_{H}^{3}},
$$


and the $\mathcal{O}\left(g_{s}^{-2}\right)$ saddle point contribution to the free energy is

$$
\frac{\ln (Z)}{V}=\frac{1}{g_{s}^{2}} \cdot \frac{\left(T-T_{H}\right)^{2}}{32 \pi^{4} \alpha^{\prime 4} T_{H}^{6} b} .
$$

On the other hand, for $b<0, S_{\text {eff }}$ is the Landau Ginzburg free energy for a system that potentially undergoes a first order phase transition at a temperature lower than $T_{H}$. The $\mathcal{O}\left(g_{s}^{0}\right)$ free energy below the phase transition temperature is once again computed by free string theory. In this case the high temperature behavior is dominated by large values of $W$, so it cannot be controlled in string perturbation theory; if there exists a high temperature saddle point, $\frac{\ln (Z)}{V}$ is again of $\mathcal{O}\left(g_{s}^{-2}\right)$, but its precise value depends on the details of the terms we denoted by "..." in (C.2).

For string theory in flat space the massless dilaton is always one of the modes $\varphi_{n}$, giving an infinite negative contribution to $b$ in (C.3), so one expects that if there is a phase transition in this case it would be of first order. Unfortunately, presumably in this case (and for any string theory with finite coupling in $\mathbb{R}^{d-1,1}$ ) no sensible high-temperature phase exists, both because there is a Jeans instability which cannot be ignored once the free energy is of order $1 / g_{s}^{2}[6]$, and because we expect the high-energy spectrum to include black holes with a density of states growing faster than exponential (see, e.g., [1]). However, while the formulas we wrote above strictly apply only to the flat space case, a similar Hagedorn behavior may be found in other spaces as well, including spaces like anti-de Sitter space where the spectrum effectively has a mass gap, and where these problems do not occur. We expect that a similar effective action would arise also in these cases ${ }^{69}$. In such cases, for example in type IIB string theory on $A d S_{5} \times S^{5}$, the arguments presented above may apply, with the value of $b$ being either positive or negative, depending on the dynamics. It turns out that the specific case of string theory on anti-de Sitter space in global coordinates corresponds to the case of $b<0$, since it is known to exhibit a first order phase transition which occurs (at small curvatures) well below the Hagedorn temperature $[48,86]$. As expected from the discussion above, this phase transition is not visible in string perturbation theory around the $A d S_{5}$ background but requires additional input.

It is interesting to note the similarity between the analysis here and the analysis of $\S 6$ of the Hagedorn transition in weakly coupled large $N$ gauge theories.

\footnotetext{
${ }^{69}$ Though, in theories that effectively have finite volume, we do not expect to find strict phase transitions at finite coupling.
} 


\section{References}

[1] O. Aharony and T. Banks, Note on the quantum mechanics of $M$ theory, JHEP 9903 (1999), 016 (1999) [arXiv:hep-th/9812237].

[2] O. Aharony, S.S. Gubser, J.M. Maldacena, H. Ooguri and Y. Oz, Large $N$ field theories, string theory and gravity, Phys. Rept. 323 (2000), 183 [arXiv:hep-th/9905111].

[3] O. Aharony, J. Marsano, S. Minwalla, K. Papadodimas and M. Van Raamsdonk, work in progress.

[4] O. Aharony and E. Witten, Anti-de Sitter space and the center of the gauge group, JHEP 9811 (1998), 018 [arXiv:hep-th/9807205].

[5] L. Alvarez-Gaume, C. Gomez, H. Liu and S. Wadia, to appear.

[6] J.J. Atick and E. Witten, The Hagedorn transition and the number of degrees of freedom of string theory, Nucl. Phys. B310 (1988), 291.

[7] V. Balasubramanian and P. Kraus, A stress tensor for anti-de Sitter gravity, Commun. Math. Phys. 208 (1999), 413 [arXiv:hep-th/9902121].

[8] T. Banks and E. Rabinovici, Finite temperature behavior of the lattice Abelian Higgs model, Nucl. Phys. B160 (1979), 349;

E.H. Fradkin and S.H. Shenker, Phase diagrams of lattice gauge theories with Higgs fields, Phys. Rev. D19 (1979), 3682.

[9] J.L. Barbon and E. Rabinovici, Closed-string tachyons and the Hagedorn transition in AdS space, JHEP 0203 (2002), 057 [arXiv:hepth/0112173].

[10] J.L. Barbon and E. Rabinovici, Remarks on black hole instabilities and closed string tachyons, Found. Phys. 33 (2003), 145 [arXiv:hepth/0211212].

[11] J.L. Barbon and E. Rabinovici, Extensivity versus holography in antide Sitter spaces, Nucl. Phys. B545 (1999), 371 (1999) [arXiv:hepth/9805143];

J.L. Barbon, I.I. Kogan and E. Rabinovici, On stringy thresholds in SYM/AdS thermodynamics, Nucl. Phys. B544 (1999), 104 [arXiv:hepth/9809033];

S.A. Abel, J.L. Barbon, I.I. Kogan and E. Rabinovici, String thermodynamics in D-brane backgrounds, JHEP 9904 (1999), 015 [arXiv:hepth/9902058];

S.A. Abel, J.L. Barbon, I.I. Kogan and E. Rabinovici, Some thermodynamical aspects of string theory, arXiv:hep-th/9911004. 
[12] K. Bardakci and C.B. Thorn, A worldsheet description of large $N_{c}$ quantum field theory, Nucl. Phys. B626 (2002), 287 [arXiv:hep-th/0110301]; C.B. Thorn, A worldsheet description of planar Yang-Mills theory, Nucl. Phys. B637 (2002), 272 [Erratum-ibid. B648 (2003), 457] [arXiv:hepth/0203167];

K. Bardakci and C.B. Thorn, A mean field approximation to the world sheet model of planar $\phi^{3}$ field theory, Nucl. Phys. B652 (2003), 196 [arXiv:hep-th/0206205];

S. Gudmundsson, C.B. Thorn and T.A. Tran, BT worldsheet for supersymmetric gauge theories, Nucl. Phys. B649 (2003), 3 [arXiv:hepth/0209102];

K. Bardakci and C.B. Thorn, An improved mean field approximation on the worldsheet for planar $\phi^{3}$ theory, Nucl. Phys. B661 (2003), 235 [arXiv:hep-th/0212254];

C.B. Thorn and T.A. Tran, The fishnet as anti-ferromagnetic phase of worldsheet Ising spins, arXiv:hep-th/0307203;

C.B. Thorn, Quantum field theory in the language of light-cone string, arXiv:hep-th/0310121;

A. Mikhailov, Notes on higher spin symmetries, arXiv:hep-th/0201019; A.A. Tseytlin, On limits of superstring in $A d S_{5} \times S^{5}$, Theor. Math. Phys. 133 (2002), 1376 [Teor. Mat. Fiz. 133 (2002), 69] [arXiv:hepth/0201112];

E. Sezgin and P. Sundell, Massless higher spins and holography, Nucl. Phys. B644 (2002), 303 [Erratum-ibid. B660 (2003), 403] [arXiv:hepth/0205131].

[13] D.S. Berman and M.K. Parikh, Confinement and the AdS/CFT correspondence, Phys. Lett. B483 (2000), 271 [arXiv:hep-th/0002031].

[14] M. Bianchi, J.F. Morales and H. Samtleben, On stringy $A d S_{5} \times S^{5}$ and higher spin holography, JHEP 0307 (2003), 062 [arXiv:hep-th/0305052].

[15] N.D. Birrell and P.C. Davies, Quantum fields in curved space.

[16] A. Brandhuber, N. Itzhaki, J. Sonnenschein and S. Yankielowicz, Wilson loops in the large $N$ limit at finite temperature, Phys. Lett. B434 (1998), 36 [arXiv:hep-th/9803137];

A. Brandhuber, N. Itzhaki, J. Sonnenschein and S. Yankielowicz, Wilson loops, confinement, and phase transitions in large $N$ gauge theories from supergravity, JHEP 9806 (1998), 001 [arXiv:hep-th/9803263].

[17] A. Buchel, Finite temperature resolution of the Klebanov-Tseytlin singularity, Nucl. Phys. B600 (2001), 219 [arXiv:hep-th/0011146];

A. Buchel, C.P. Herzog, I.R. Klebanov, L.A. Pando Zayas and 
A.A. Tseytlin, Non-extremal gravity duals for fractional D3-branes on the conifold, JHEP 0104, 033 (2001) [arXiv:hep-th/0102105];

S. S. Gubser, C. P. Herzog, I. R. Klebanov and A. A. Tseytlin, Restoration of chiral symmetry: A supergravity perspective, JHEP 0105 (2001), 028 [arXiv:hep-th/0102172].

[18] A. Buchel and A.R. Frey, Comments on supergravity dual of pure $N=1$ super Yang Mills theory with unbroken chiral symmetry, Phys. Rev. D64 (2001), 064007 [arXiv:hep-th/0103022];

A. Buchel, On the thermodynamic instability of LST, arXiv:hepth/0107102;

S.S. Gubser, A.A. Tseytlin and M.S. Volkov, Non-Abelian 4-d black holes, wrapped 5-branes, and their dual descriptions, JHEP 0109 (2001), 017 [arXiv:hep-th/0108205].

[19] A. Buchel and L.A. Pando Zayas, Hagedorn vs. Hawking-Page transition in string theory, Phys. Rev. D68 (2003), 066012 [arXiv:hepth/0305179].

[20] C.P. Burgess, N.R. Constable and R.C. Myers, The free energy of $N=4$ Super Yang-Mills and the AdS/CFT correspondence, JHEP 9908 (1999), 017 [arXiv:hep-th/9907188].

[21] N. Cabibbo and G. Parisi, Exponential hadronic spectrum and quark liberation, Phys. Lett. B59 (1975), 67.

[22] A. Chamblin, R. Emparan, C.V. Johnson and R.C. Myers, Charged AdS black holes and catastrophic holography, Phys. Rev. D60 (1999), 064018 [arXiv:hep-th/9902170];

M. Cvetic and S.S. Gubser, Phases of R-charged black holes, spinning branes and strongly coupled gauge theories, JHEP 9904 (1999), 024 [arXiv:hep-th/9902195];

A. Chamblin, R. Emparan, C.V. Johnson and R.C. Myers, Holography, thermodynamics and fluctuations of charged AdS black holes, Phys. Rev. D60 (1999, 104026 [arXiv:hep-th/9904197].

[23] A. Clark, A. Karch, P. Kovtun and D. Yamada, Construction of bosonic string theory on infinitely curved anti-de Sitter space, Phys. Rev. D 68 (2003), 066011 [arXiv:hep-th/0304107].

[24] J.C. Collins and M.J. Perry, Superdense matter: neutrons or asymptotically free quarks?, Phys. Rev. Lett. 34 (1975), 1353.

[25] N.R. Constable, D.Z. Freedman, M. Headrick, S. Minwalla, L. Motl, A. Postnikov and W. Skiba, PP-wave string interactions from perturbative Yang-Mills theory, JHEP 0207 (2002), 017 [arXiv:hep-th/0205089]; 
C. Kristjansen, J. Plefka, G.W. Semenoff and M. Staudacher, A new double-scaling limit of $N=4$ super Yang-Mills theory and PP-wave strings, Nucl. Phys. B643 (2002), 3 [arXiv:hep-th/0205033];

N. Beisert, C. Kristjansen, J. Plefka, G.W. Semenoff and M. Staudacher, $B M N$ correlators and operator mixing in $N=4$ super Yang-Mills theory, Nucl. Phys. B650 (2003), 125 [arXiv:hep-th/0208178];

N.R. Constable, D.Z. Freedman, M. Headrick and S. Minwalla, Operator mixing and the BMN correspondence, JHEP 0210 (2002), 068 [arXiv:hep-th/0209002];

D.J. Gross, A. Mikhailov and R. Roiban, Operators with large $R$ charge in $N=4$ Yang-Mills theory, Annals Phys. 301 (2002), 31 [arXiv:hepth/0205066];

R. de Mello Koch, A. Jevicki and J.P. Rodrigues, Collective string field theory of matrix models in the BMN limit, arXiv:hep-th/0209155;

N. Beisert, C. Kristjansen, J. Plefka and M. Staudacher, BMN gauge theory as a quantum mechanical system, Phys. Lett. B558 (2003), 229 [arXiv:hep-th/0212269];

J. Gomis, S. Moriyama and J.W. Park, SYM description of SFT Hamiltonian in a pp-wave background, arXiv:hep-th/0210153;

J. Gomis, S. Moriyama and J.W. Park, SYM description of pp-wave string interactions: Singlet sector and arbitrary impurities, Nucl. Phys. B665 (2003), 49 [arXiv:hep-th/0301250];

R. de Mello Koch, A. Donos, A. Jevicki and J. P. Rodrigues, Derivation of string field theory from the large N BMN limit, Phys. Rev. D68 (2003), 065012 [arXiv:hep-th/0305042].

[26] J.R. David, G. Mandal and S.R. Wadia, Microscopic formulation of black holes in string theory, Phys. Rept. 369 (2002), 549 [arXiv:hepth/0203048].

[27] P. de Medeiros and S.P. Kumar, Spacetime Virasoro algebra from strings on zero radius $A d S_{3}$, arXiv:hep-th/0310040.

[28] A. Dhar, G. Mandal and S.R. Wadia, String bits in small radius AdS and weakly coupled $N=4$ super Yang-Mills theory, I, arXiv:hep-th/0304062.

[29] R. Dijkgraaf, J.M. Maldacena, G.W. Moore and E. Verlinde, A black hole farey tail, arXiv:hep-th/0005003.

[30] A. Fotopoulos and T.R. Taylor, Comment on two-loop free energy in $N=4$ supersymmetric Yang-Mills theory at finite temperature, Phys. Rev. D59 (1999), 061701 [arXiv:hep-th/9811224].

[31] D.Z. Freedman and J.A. Minahan, Finite temperature effects in the supergravity dual of the $N=1^{*}$ gauge theory, JHEP 0101 (2001), 036 
[arXiv:hep-th/0007250];

A. Buchel and J.T. Liu, Thermodynamics of the $N=2^{*}$ flow, arXiv:hepth/0305064.

[32] K. Furuuchi, E. Schreiber and G.W. Semenoff, Five-brane thermodynamics from the Matrix model, arXiv:hep-th/0310286.

[33] C.R. Gattringer, L.D. Paniak and G.W. Semenoff, Deconfinement transition for quarks on a line, Annals Phys. 256 (1997), 74 [arXiv:hepth/9612030].

[34] A. Gocksch and R.D. Pisarski, Partition function for the eigenvalues of the Wilson line, Nucl. Phys. B402 (1993), 657 [arXiv:hep-ph/9302233].

[35] Y.Y. Goldschmidt, $1 / N$ expansion in two-dimensional lattice gauge theory, J. Math. Phys. 21 (1980), 1842.

[36] R. Gopakumar, From free fields to AdS, arXiv:hep-th/0308184.

[37] R. Gopakumar and C. Vafa, On the gauge theory/geometry correspondence, Adv. Theor. Math. Phys. 3 (1999), 1415 [arXiv:hep-th/9811131].

[38] R. Gregory and R. Laflamme, Black strings and p-branes are unstable, Phys. Rev. Lett. 70 (1993), 2837 [arXiv:hep-th/9301052].

[39] D.J. Gross, Two-dimensional QCD as a string theory, Nucl. Phys. B400 (1993), 161 [arXiv:hep-th/9212149];

D.J. Gross and W.I. Taylor, Two-dimensional QCD is a string theory, Nucl. Phys. B400 (1993), 181 [arXiv:hep-th/9301068];

D.J. Gross and W.I. Taylor, Twists and Wilson loops in the string theory of two-dimensional QCD, Nucl. Phys. B403 (1993), 395 [arXiv:hepth/9303046].

[40] D.J. Gross, R.D. Pisarski and L.G. Yaffe, $Q C D$ and instantons at finite temperature, Rev. Mod. Phys. 53 (1981), 43.

[41] D.J. Gross and E. Witten, Possible third order phase transition in the large $N$ lattice gauge theory, Phys. Rev. D21 (1980), 446 (1980);

S. Wadia, A study of $U(N)$ lattice gauge theory in two-dimensions, preprint EFI-79/44-CHICAGO;

S.R. Wadia, $N=\infty$ phase transition in a class of exactly soluble model lattice gauge theories, Phys. Lett. B93 (1980), 403.

[42] S.S. Gubser, Thermodynamics of spinning D3-branes, Nucl. Phys. B 551 (1999), 667 [arXiv:hep-th/9810225];

P. Kraus, F. Larsen and S. P. Trivedi, The Coulomb branch of gauge 
theory from rotating branes, JHEP 9903 (1999), 003 [arXiv:hepth/9811120].

[43] S.S. Gubser, I.R. Klebanov and A.A. Tseytlin, Coupling constant dependence in the thermodynamics of $N=4$ supersymmetric Yang-Mills theory, Nucl. Phys. B534 (1998), 202 [arXiv:hep-th/9805156].

[44] R. Hagedorn, Statistical thermodynamics of strong interactions at highenergies, Nuovo Cim. Suppl. 3 (1965), 147.

[45] P. Haggi-Mani and B. Sundborg, Free large $N$ supersymmetric YangMills theory as a string theory, JHEP 0004 (2000), 031 [arXiv:hepth/0002189];

B. Sundborg, Stringy gravity, interacting tensionless strings and massless higher spins, Nucl. Phys. Proc. Suppl. 102 (2001), 113 [arXiv:hepth/0103247].

[46] J. Hallin and D. Persson, Thermal phase transition in weakly interacting, large $N_{c}$ QCD, Phys. Lett. B429 (1998), 232 [arXiv:hep$\mathrm{ph} / 9803234]$.

[47] M.B. Halpern, On the large $N$ limit of conformal field theory, Annals Phys. 303 (2003), 321 [arXiv:hep-th/0208150].

[48] S.W. Hawking and D.N. Page, Thermodynamics of black holes in antide Sitter space, Commun. Math. Phys. 87 (1983), 577.

[49] S.W. Hawking and H.S. Reall, Charged and rotating AdS black holes and their CFT duals, Phys. Rev. D61 (2000), 024014 [arXiv:hepth/9908109].

[50] J. Jurkiewicz and K. Zalewski, Vacuum structure of the $U(N \rightarrow \infty)$ gauge theory on a two-dimensional lattice for a broad class of variant actions, Nucl. Phys. B220 (1983), 167.

[51] A. Karch, Lightcone quantization of string theory duals of free field theories, arXiv:hep-th/0212041.

[52] C.J. Kim and S.J. Rey, Thermodynamics of large- $N$ super Yang-Mills theory and AdS/CFT correspondence, Nucl. Phys. B564 (2000), 430 [arXiv:hep-th/9905205].

[53] I.R. Klebanov and E. Witten, Superconformal field theory on threebranes at a Calabi-Yau singularity, Nucl. Phys. B536 (1998), 199 [arXiv:hepth/9807080];

I.R. Klebanov and N.A. Nekrasov, Gravity duals of fractional branes 
and logarithmic $R G$ flow, Nucl. Phys. B574 (2000), 263 [arXiv:hepth/9911096];

I.R. Klebanov and A.A. Tseytlin, Gravity duals of supersymmetric $S U(N) \times S U(N+M)$ gauge theories, Nucl. Phys. B578 (2000), 123 [arXiv:hep-th/0002159];

I.R. Klebanov and M.J. Strassler, Supergravity and a confining gauge theory: Duality cascades and $\chi S B$-resolution of naked singularities, JHEP 0008 (2000), 052 [arXiv:hep-th/0007191].

[54] D. Kutasov, Two-dimensional QCD coupled to adjoint matter and string theory, Nucl. Phys. B414 (1994), 33 [arXiv:hep-th/9306013].

[55] D. Kutasov and F. Larsen, Partition sums and entropy bounds in weakly coupled CFT, JHEP 0101 (2001), 001 [arXiv:hep-th/0009244].

[56] K. Landsteiner and E. Lopez, The thermodynamic potentials of KerrAdS black holes and their CFT duals, JHEP 9912 (1999), 020 [arXiv:hep-th/9911124].

[57] M. Li, E.J. Martinec and V. Sahakian, Black holes and the SYM phase diagram, Phys. Rev. D59 (1999), 044035 [arXiv:hep-th/9809061];

E.J. Martinec and V. Sahakian, Black holes and the SYM phase diagram, II, Phys. Rev. D59 (1999), 124005 [arXiv:hep-th/9810224].

[58] B. Lucini, M. Teper and U. Wenger, The high temperature phase transition in $S U(N)$ gauge theories, arXiv:hep-lat/0307017.

[59] J.M. Maldacena, Wilson loops in large $N$ field theories, Phys. Rev. Lett. 80 (1998), 4859 [arXiv:hep-th/9803002].

[60] J.M. Maldacena, The large $N$ limit of superconformal field theories and supergravity, Adv. Theor. Math. Phys. 2 (1998), 231 [Int. J. Theor. Phys. 38, 1113 (1999)] [arXiv:hep-th/9711200];

S.S. Gubser, I.R. Klebanov and A.M. Polyakov, Gauge theory correlators from non-critical string theory, Phys. Lett. B428 (1998), 105 [arXiv:hep-th/9802109];

E. Witten, Anti-de Sitter space and holography, Adv. Theor. Math. Phys. 2 (1998), 253 [arXiv:hep-th/9802150].

[61] J.M. Maldacena and C. Nunez, Towards the large $N$ limit of pure $N=1$ super Yang Mills, Phys. Rev. Lett. 86 (2001), 588 [arXiv:hepth/0008001].

[62] J. Maldacena, M.M. Sheikh-Jabbari and M. Van Raamsdonk, Transverse fivebranes in matrix theory, JHEP 0301 (2003), 038 [arXiv:hepth/0211139]. 
[63] J.A. Minahan and K. Zarembo, The Bethe-ansatz for $N=4$ super Yang-Mills, JHEP 0303 (2003), 013 [arXiv:hep-th/0212208];

N. Beisert, C. Kristjansen and M. Staudacher, The dilatation operator of $N=4$ super Yang-Mills theory, Nucl. Phys. B664 (2003), 131 [arXiv:hep-th/0303060];

G. Mandal, N.V. Suryanarayana and S.R. Wadia, Aspects of semiclassical strings in $A d S_{5}$, Phys. Lett. B543 (2002), 81 [arXiv:hepth/0206103];

I. Bena, J. Polchinski and R. Roiban, Hidden symmetries of the $A d S_{5} \times S^{5}$ superstring, arXiv:hep-th/0305116;

$\mathrm{N}$. Beisert, The complete one-loop dilatation operator of $N=4$ super Yang-Mills theory, arXiv:hep-th/0307015;

N. Beisert and M. Staudacher, The $N=4$ SYM integrable super spin chain, Nucl. Phys. B670 (2003), 439 [arXiv:hep-th/0307042];

L. Dolan, C.R. Nappi and E. Witten, A relation between approaches to integrability in superconformal Yang-Mills theory, JHEP 0310 (2003), 017 [arXiv:hep-th/0308089];

L.F. Alday, Nonlocal charges on $A d S_{5} \times S^{5}$ and pp-waves, arXiv:hepth/0310146;

T. Klose and J. Plefka, On the integrability of large $N$ plane-wave matrix theory, arXiv:hep-th/0310232.

[64] R. Narayanan and H. Neuberger, Large $N$ reduction in continuum, Phys. Rev. Lett. 91 (2003), 081601 [arXiv:hep-lat/0303023];

J. Kiskis, R. Narayanan and H. Neuberger, Does the crossover from perturbative to nonperturbative physics in $Q C D$ become a phase transition at infinite $N$ ?, Phys. Lett. B574 (2003), 65 [arXiv:hep-lat/0308033].

[65] H. Ooguri and C. Vafa, Worldsheet derivation of a large $N$ duality, Nucl. Phys. B641 (2002), 3 [arXiv:hep-th/0205297].

[66] L.A. Pando Zayas and D. Vaman, Strings in $R R$ plane wave background at finite temperature, Phys. Rev. D67 (2003), 106006 [arXiv:hepth/0208066];

B.R. Greene, K. Schalm and G. Shiu, On the Hagedorn behaviour of ppwave strings and $N=4 S Y M$ theory at finite $R$-charge density, Nucl. Phys. B652 (2003), 105 [arXiv:hep-th/0208163];

Y. Sugawara, Thermal amplitudes in $D L C Q$ superstrings on pp-waves, Nucl. Phys. B650 (2003), 75 [arXiv:hep-th/0209145];

R.C. Brower, D.A. Lowe and C.I. Tan, Hagedorn transition for strings on pp-waves and tori with chemical potentials, Nucl. Phys. B652 (2003), 127 [arXiv:hep-th/0211201];

Y. Sugawara, Thermal partition function of superstring on compactified pp-wave, Nucl. Phys. B661 (2003), 191 [arXiv:hep-th/0301035]; 
G. Grignani, M. Orselli, G.W. Semenoff and D. Trancanelli, The superstring Hagedorn temperature in a pp-wave background, JHEP 0306 (2003), 006 [arXiv:hep-th/0301186];

S.J. Hyun, J.D. Park and S.H. Yi, Thermodynamic behavior of IIA string theory on a pp-wave, arXiv:hep-th/0304239;

F. Bigazzi and A.L. Cotrone, On zero-point energy, stability and Hagedorn behavior of type IIB strings on pp-waves, JHEP 0308 (2003), 052 [arXiv:hep-th/0306102];

L.A. Pando Zayas and D. Vaman, Hadronic density of states from string theory, Phys. Rev. Lett. 91 (2003), 111602 [arXiv:hep-th/0306107];

R. Apreda, F. Bigazzi and A.L. Cotrone, Strings on pp-waves and hadrons in (softly broken) $N=1$ gauge theories, arXiv:hep-th/0307055.

[67] R.D. Pisarski, Finite temperature QCD at large $N$, Phys. Rev. D29 (1984), 1222.

[68] R.D. Pisarski and M. Tytgat, Why the SU(infinity) deconfining phase transition might be of second order, arXiv:hep-ph/9702340.

[69] J. Polchinski, High temperature limit of the confining phase, Phys. Rev. Lett. 68 (1992), 1267 [arXiv:hep-th/9109007].

[70] J. Polchinski, unpublished.

[71] A.M. Polyakov, Thermal properties of gauge fields and quark liberation, Phys. Lett. B72 (1978), 477.

[72] A.M. Polyakov, String theory and quark confinement, Nucl. Phys. Proc. Suppl. 68 (1998), 1 [arXiv:hep-th/9711002] and references therein.

[73] A.M. Polyakov, Gauge fields and space-time, Int. J. Mod. Phys. A17S1 (2002), 119 [arXiv:hep-th/0110196].

[74] S.J. Rey, S. Theisen and J.T. Yee, Wilson-Polyakov loop at finite temperature in large $N$ gauge theory and anti-de Sitter supergravity, Nucl. Phys. B527 (1998), 171 [arXiv:hep-th/9803135].

[75] S.-J. Rey and J. Yee, Macroscopic strings as heavy quarks in large $N$ gauge theories and anti-de Sitter supergravity, Eur. Phys. J. C22 (2001), 379 [arXiv:hep-th/9803001].

[76] B. Sathiapalan, Vortices on the string world sheet and constraints on toral compactification, Phys. Rev. D35, 3277 (1987);

Y.I. Kogan, Vortices on the world sheet and string's critical dynamics, JETP Lett. 45 (1987), 709 [Pisma Zh. Eksp. Teor. Fiz. 45, 556 (1987)]. 
[77] B. Sundborg, The Hagedorn transition, deconfinement and $N=4 S Y M$ theory, Nucl. Phys. B573 (2000), 349 [arXiv:hep-th/9908001].

[78] L. Susskind, Lattice models of quark confinement at high temperature, Phys. Rev. D20 (1979), 2610.

[79] B. Svetitsky and L.G. Yaffe, Critical behavior at finite temperature confinement transitions, Nucl. Phys. B210 (1982), 423.

[80] G. 't Hooft, A planar diagram theory for strong interactions, Nucl. Phys. B72 (1974), 461.

[81] G. 't Hooft, On the phase transition towards permanent quark confinement, Nucl. Phys. B138 (1978), 1.

[82] G. 't Hooft, Under the spell of the gauge principle, Adv. Ser. Math. Phys. 19 (1994), 1.

[83] G. 't Hooft, A two-dimensional model for mesons, Nucl. Phys. B75 (1974), 461;

S. Dalley and I.R. Klebanov, String spectrum of $(1+1)$-dimensional large $N$ QCD with adjoint matter, Phys. Rev. D47 (1993), 2517 [arXiv:hepth/9209049];

G. Bhanot, K. Demeterfi and I.R. Klebanov, $(1+1)$-dimensional large $N$ QCD coupled to adjoint fermions, Phys. Rev. D48 (1993), 4980 [arXiv:hep-th/9307111];

K. Demeterfi, I.R. Klebanov and G. Bhanot, Glueball spectrum in $a(1+1)$-dimensional model for QCD, Nucl. Phys. B418 (1994), 15 [arXiv:hep-th/9311015];

I.I. Kogan and A.R. Zhitnitsky, Two dimensional QCD with matter in adjoint representation: What does it teach us?, Nucl. Phys. B465 (1996), 99 [arXiv:hep-ph/9509322].

[84] C.B. Thorn, Infinite $N_{c} Q C D$ at finite temperature: is there an ultimate temperature?, Phys. Lett. B99 (1981), 458.

[85] A.A. Tseytlin, On limits of superstring in $A d S_{5} \times S^{5}$, Theor. Math. Phys. 133 (2002), 1376 [Teor. Mat. Fiz. 133 (2002), 69] [arXiv:hepth/0201112].

[86] E. Witten, Anti-de Sitter space, thermal phase transition, and confinement in gauge theories, Adv. Theor. Math. Phys. 2 (1998), 505 [arXiv:hep-th/9803131].

[87] L.G. Yaffe and B. Svetitsky, First order phase transition in the SU(3) gauge theory at finite temperature, Phys. Rev. D26 (1982), 963. 